Portland State University

PDXScholar

Summer 8-31-2017

\title{
Development of the Global-Self Through Collegiate Recreational Sports
}

Alexander Rocco Accetta

Portland State University

Follow this and additional works at: https://pdxscholar.library.pdx.edu/open_access_etds

Part of the Higher Education Commons, and the Sports Studies Commons Let us know how access to this document benefits you.

\section{Recommended Citation}

Accetta, Alexander Rocco, "Development of the Global-Self Through Collegiate Recreational Sports" (2017). Dissertations and Theses. Paper 3807.

https://doi.org/10.15760/etd.5691

This Dissertation is brought to you for free and open access. It has been accepted for inclusion in Dissertations and Theses by an authorized administrator of PDXScholar. Please contact us if we can make this document more accessible: pdxscholar@pdx.edu. 
Development of the Global-Self Through Collegiate Recreational Sports

\title{
by
}

Alexander Rocco Accetta

A dissertation submitted in partial fulfillment of the requirements for the degree of

\section{Doctor of Education}

in

Educational Leadership: Postsecondary Education

\author{
Dissertation Committee: \\ Karen Haley, Chair \\ Candyce Reynolds \\ Brian Janssen \\ Shawn Smallman
}

Portland State University

2017 
(C) 2017 Alexander Rocco Accetta 


\begin{abstract}
Today's student has more access to global issues than any previous generation. Nearly one million higher education students study abroad worldwide, the workplace reflects a need to be interculturally competent, and students rarely have opportunities to learn how to thrive in the new global environment. This study explored how higher education, and specifically collegiate recreation, is responding to this reality. The development of Killick's global-self is a guiding theme and was used to investigate how students perceive the development of their global-self after experiencing interventions designed to introduce the concepts of internationalization and globalization into a collegiate recreation intramural program. The study also examines how students experience the interventions. The literature review focuses on institutional perspective, student engagement, learning theory, the role of sport and recreation in social change, and collegiate recreation professional competencies. A bounded 8-week case study, using aspects of community action research, was the research method. Observations, surveys, and semi-structured interviews provide data on students' perceptions of their development of a global-self and their experience in the designed intramural program. Themes identified in the data provide evidence that the interventions helped students develop both their sense of self-in-the-world and their ability to act-in-the-world. The data suggest that students found value in the interventions and believed them to be a worthwhile addition to the intramural program. Finally, the results of the study suggest that similar interventions can be applied to multiple areas in collegiate recreation and potentially expanded to other forms of co-curricular activities.
\end{abstract}




\section{Table of Contents}

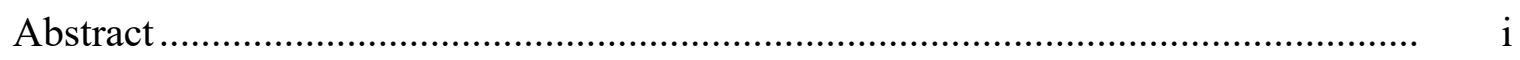

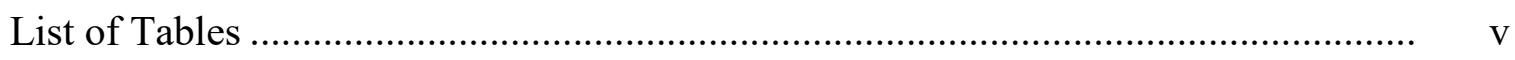

CHAPTER 1: INTRODUCTION .................................................................

Background of the Problem ......................................................................... 2

Internationalization and Globalization..................................................... 3

Intercultural Competence ....................................................................... 7

The Role of Sport and Leisure .............................................................. 8

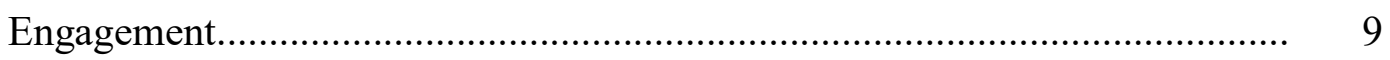

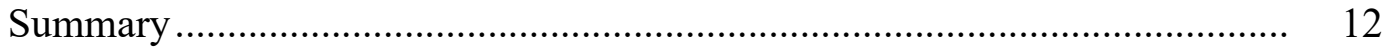

Statement of the Research Problem ........................................................... 12

Purpose of the Study ............................................................................. 14

Significance of the Research.......................................................................... 15

Research Methodology and Research Questions ............................................ 17

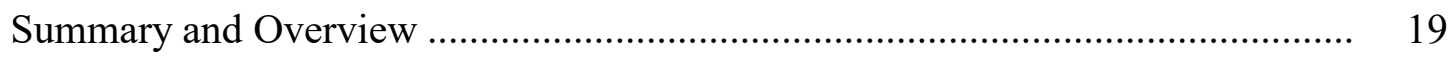

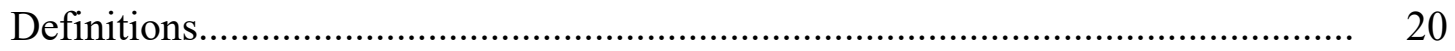

CHAPTER 2: LITERATURE REVIEW ........................................................... 22

Killick's Global-Self Theory …............................................................... 22

Institutional Perspective ....................................................................... 24

Shaping the Environment ................................................................ 25

Shaping the Curriculum ....................................................................... 28

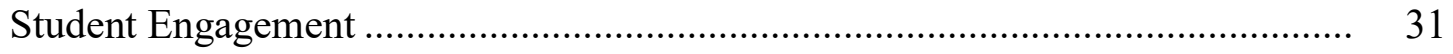

Astin and Student Development ............................................................ $\quad 32$

Tinto and Integration...................................................................... 33

Chickering and Reisser .................................................................. 35

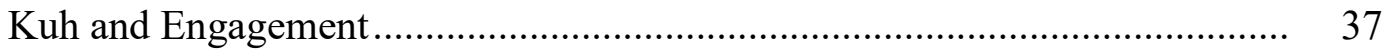

Criticisms of Student Engagement Theory ................................................ 38

Intercultural Development .................................................................... 40

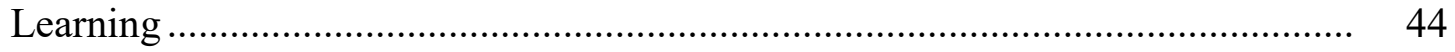

Experiential Learning..................................................................... 44 
DEVELOPMENT OF THE GLOBAL-SELF iii

Constructivist Learning..................................................................... 47

Learning at Home .................................................................................. 49

Sport and Recreation for Social Change ...................................................... 51

Sport and Social Change ...................................................................... 51

Sport and the Other .............................................................................. 54

Collegiate Recreation and International Students................................... 57

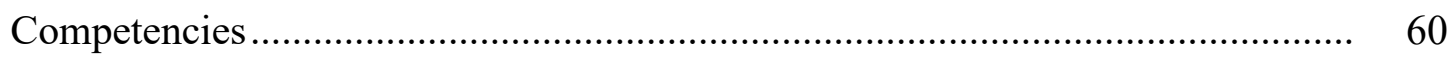

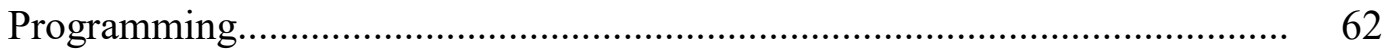

Philosophy and Theory ................................................................... 62

Personal and Professional Qualities....................................................... 63

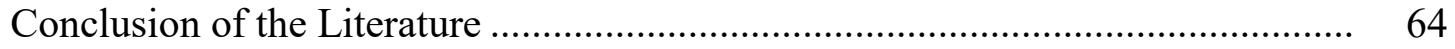

CHAPTER 3: METHODS ............................................................................ 66

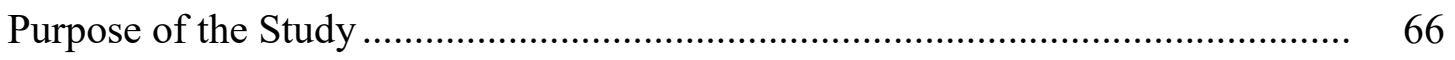

Research Perspective ................................................................................ 67

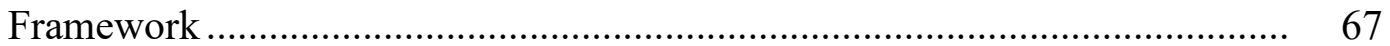

Case Study and Community Action Research ........................................ 69

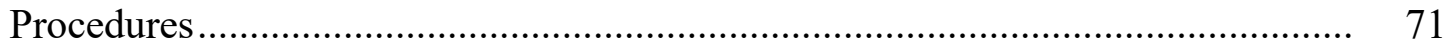

Study Context and Target Population ......................................................... 71

Research Procedures ........................................................................... 72

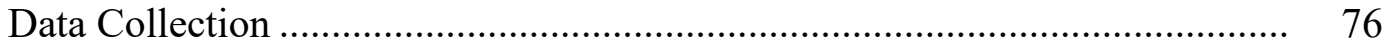

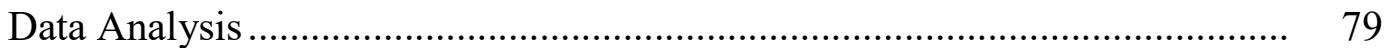

Participant and Data Care .............................................................. 83

Role of the Researcher .......................................................................... 84

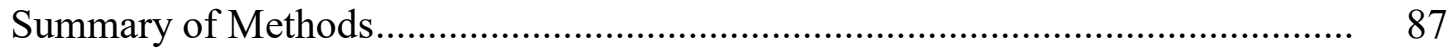

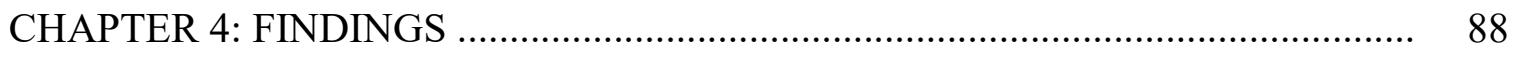

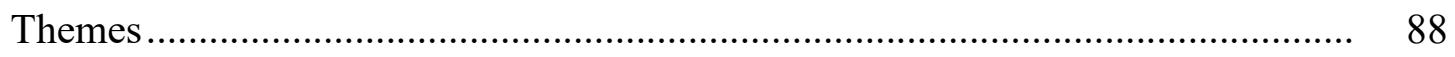

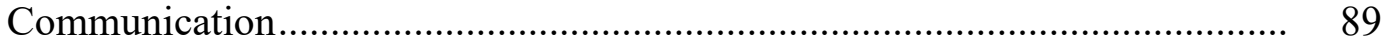

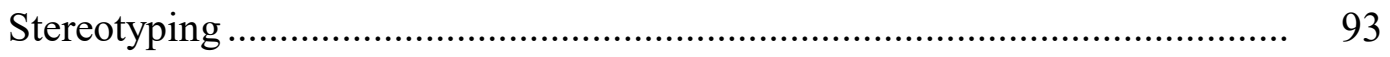

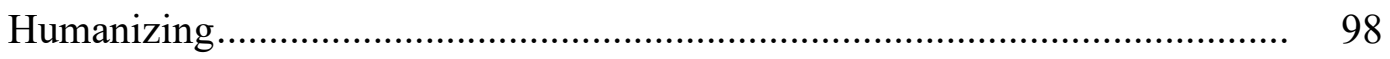

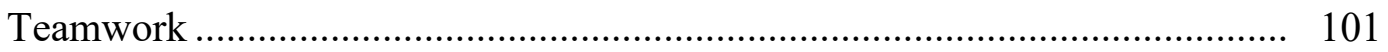


DEVELOPMENT OF THE GLOBAL-SELF _ iv

Communities .................................................................................. 103

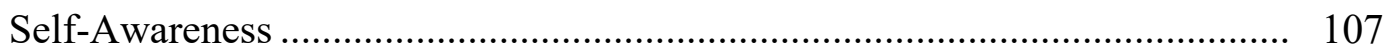

Evaluative Review .................................................................................. 109

Pre- and Post-Game Interventions .................................................. 110

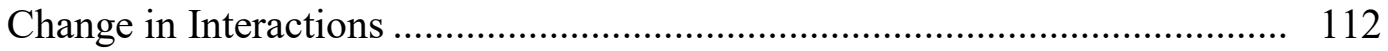

Overall Experience....................................................................... 112

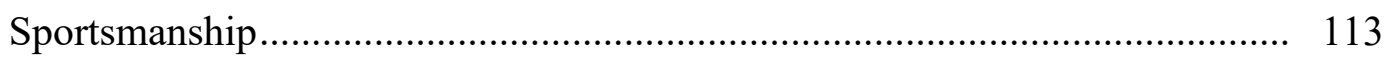

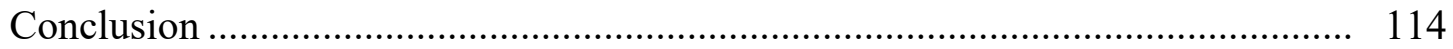

CHAPTER 5: DISCUSSION AND IMPLICATIONS ....................................... 116

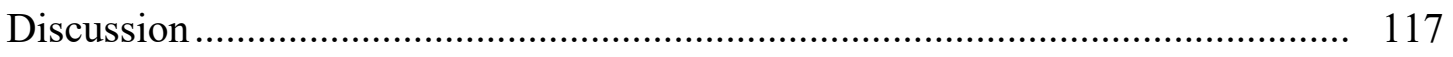

Developing the Global-Self ................................................................ 118

Developing the Program .............................................................. 127

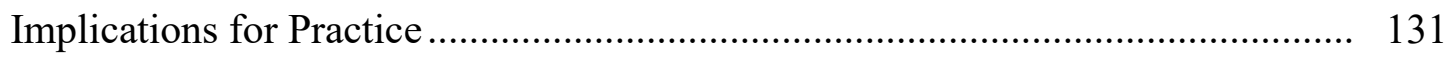

Recommendations for Improving Interventions ........................................ 132

Implications for the Professional Competency Areas............................... 134

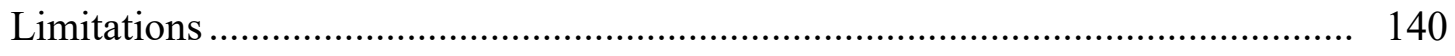

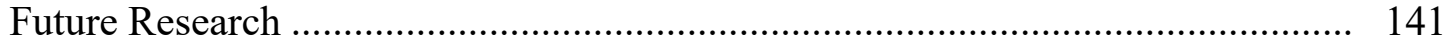

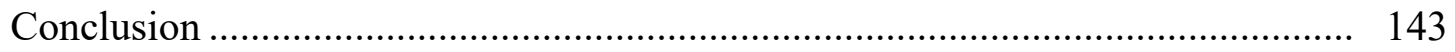

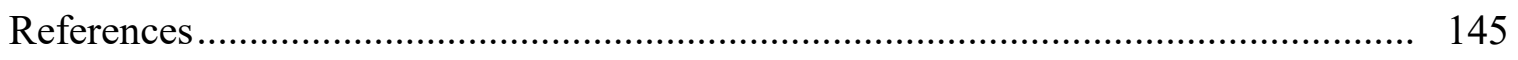

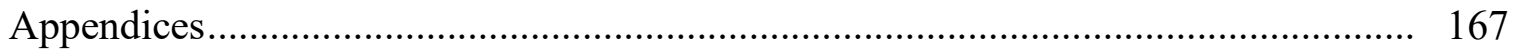

A. NIRSA Core Competencies .................................................................... 167

B. Fall 2016 Research Timeline................................................................... 172

C. Global-Self Training for Intramural Staff ............................................... 173

D. Pre-Game Script and Questions …..................................................... 176

E. Questions for Post-Game Interventions................................................ 177

F. Protocol for Online Survey .................................................................... 178

G. Informed Consent Form .................................................................... 180

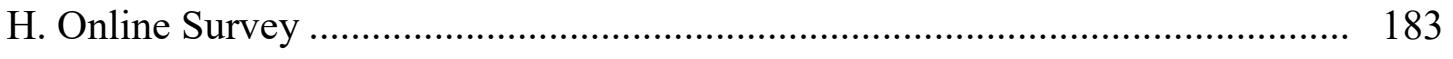

I. Semi-Structured Interview Protocol ..................................................... 187

J. Survey Response Demographics ............................................................. 190 
List of Tables

Table 1. Mature Level Attributes of Intercultural Development ............................... 41

Table 2. In-Depth Interview Participant Demographics ........................................ 79

Table 3. Player and Employee Response to Pre- and Post-Game Interventions ......... 111

Table 4. Sportsmanship Ratings for Intramural Leagues from 2015 and 2016 .......... 114 


\section{CHAPTER 1: INTRODUCTION}

Globalization has changed higher education. Geo-politics spill over national borders, information gets shared instantly with a keyboard click, and universities are opening campuses in multiple countries. There are over one million students worldwide participating in travel abroad opportunities annually, magnifying the effects of these changes. These students, often referred to as international students regardless of their place of origin, present an opportunity in higher education to increase students' global awareness.

Killick (2015) suggests that to increase global awareness, higher education would be best served by actively creating university environments where all students are supported in the development of the global-self. The global-self, Killick proposes, has two components. The first component is a student's

sense of self-in-the-world, [that the others with whom I coexist are equally human] as the primary dimension of the global-self, and precisely because this may not come to us naturally, it is the primary objective of internationalization . . . it is about global selves not citizens; connections between individuals. (Killick, 2015, p. 31)

The second component of the global-self is the student's ability to act-in-the-world, which requires the "knowledge, skills, and values which enable [the student] to act-inthe-world; by which [students have] the capabilities to take deliberative action with appropriate references to the contexts and participants implicated in the action" (Killick, 2015, p. 31). Killick (2015) maintains that the development of the global-self is integral to developing a socially just world:

... that to make their way in the increasingly interconnected and diverse globalizing world, and to do so in ways which are commensurate within a broad 
agenda of social justice, our students need opportunities to experience being global students in order to develop their capabilities as global selves. (p. 32) Multiple factors at the university influence the opportunities to develop those capabilities. The curricula, both formal and informal, is the most conventional place to work on the global-self in higher education. However, additional elements such as the university's environment, institutional level of support, and student interests, also contribute to students' development of the global-self.

Globalization and internationalization have influenced a new paradigm for much of higher education and have shaped both the environment and the curricula. Due in part to this new paradigm, intercultural competence has become an important component of student development. The support for the development of intercultural competence, offered in a co-curricular context, is at the core of this study. Understanding how and why the co-curricular context is important to the development of the global-self is accomplished in this chapter by describing the background of the problem-in-practice. Collegiate recreation, and particularly intramural sports, is introduced as a particularly helpful co-curricular activity to help develop the global-self. After a discussion of the problem-in-practice, this chapter highlights the research problem, describes the purpose of the study and the significance of the research, and closes with a brief introduction to the research methodology and questions.

\section{Background of Problem}

Four aspects of the problem-in-practice highlight how collegiate recreation can help the development of the global-self. First, background information on globalization and internationalization is shared to place the changing university dynamics in perspective. Second, an introduction to the role of sports and leisure in social change is 
introduced as a powerful tool to champion and model social justice. Third, a discussion on student engagement on campus helps demonstrate why co-curricular activities are important to this topic. Finally, a review of intercultural competence introduces an important tool and concept to help define and measure the global-self.

\section{Internationalization and Globalization}

In the new paradigm of global connectedness, it is important that students learn how to operate in a highly internationalized environment. Unfortunately, higher education institutions rarely have policies and practices that support students developing globally competent perspectives (Stier, 2004). While aspects of globalization appear around campus every day — we drive Japanese cars, type on computers made in China, and eat grapes from Chile-its influence on campus often lacks institutional and departmental focus. There are not enough structured and intentionally designed cocurricular opportunities at universities to create the situations where students can create their global-self (Killick, 2013). The development of the global-self is critical if we hope to create a more socially just world through higher education. The internationalization of campuses is one tool that is being tried to improve these outcomes (Childress, 2009; Croom, 2014; Hser, 2005).

The term internationalization has gone through many different iterations (De Wit, 2002; Söderqvist, 2007), but is generally used to define a range of tools used to address the interactions between international and domestic students on campus. The tools supporting campus internationalization are broad, ranging from admissions decisions, to classroom curriculum, to the out-of-classroom experience, each attempting to integrate intercultural dimensions into the purpose and function of the learning process (Knight, 
2004; Wachter, 2003). Internationalization can also describe the impact on the individual's experience of these interactions. For this paper, internationalization will refer to the range of activities, policies, and practices universities use to improve intercultural interactions, especially between international students and domestic students, staff, and faculty.

Four rationales can be used to justify internationalization, including politics, economics, academics, and sociocultural reasons (Childress, 2009; Knight \& De Wit, 1997). The political and policy history of internationalization in higher education is most often attributed to Russia's successful launch of Sputnik in 1957 (Cross, 2014; Hser, 2005). This satellite's launch challenged America's leadership position, at least symbolically, and created a number of political rationales for increasing students' knowledge of foreign policy and national security (Childress, 2009; Knight, 2004). Economically, two factors intertwine to make internationalization important. First, universities are relying on international students paying full out-of-state tuition to help cover the growing costs associated with less governmental subsidy (Qiang, 2003). Second, there is evidence that completing higher education is a gateway to higher earnings and career success regardless of nationality (Bruner \& Iannarelli, 2011). Academically, internationalization is viewed as an essential component of students' development, particularly to strengthen liberal education, increase understanding, and develop global thinking (Chickering \& Braskamp, 2009). Sociocultural reasons for internationalization include developing "students' intercultural communication skills ... to address cultural and ethnic diversity within and between countries" (Childress, 2009, p. 290). The four rationales have a reciprocal effect, creating an upward spiral that creates 
more interest in internationalization (Knight \& De Wit, 1997). This growing interest manifested in nearly one million students studying abroad during the 2012-2013 academic year (Institute on International Education [IIE], 2014), almost double the number 20 years earlier.

Contrasted with internationalization, globalization in higher education describes the efforts of an institution to engage in a world where international borders are no longer barriers to the exchange of information. According to Knight and De Wit (1997), globalization is defined as "the flow of technology, economy, knowledge, people, values, [and] ideas ... across borders. Globalization affects each country in a different way due to a nation's individual history, traditions, culture and priorities" (p. 6). Globalization is thus less focused on the impact on the individual and more on the impact on institutional relationships. Globalization is not without its critics. Some postcolonial theorists argue that globalization resulted in a less diverse world as the United States has subjugated foreign lands, created greater inequalities, destroyed ecological systems, and initiated more homogenization of the world (Burney, 2012; Featherstone, 1990). Regardless of the current political environment, globalization is "part of the environment in which the international dimensions of higher education is becoming more important and significantly changing” (Knight, 2004, p. 8). In this paper, globalization will include the strategies and initiatives higher education institutions use, including activities designed to engage across international borders and to produce communities that embrace the importance of internationalization.

Globalization has changed university operations, impacting the way knowledge is created and distributed (Altbach \& Knight, 2007). Recruiting international students to 
American campuses, along with study abroad programs for domestic students, are two of the main strategies that higher education institutions use to prepare students for a new global world (Ho, Bulman-Fleming, \& Mitchell, 2003). These strategies have resulted in higher education, as a collective, playing an integral part in rethinking the social, cultural, and economic roles that it can take to influence a globalizing world (Enders, 2004).

Despite the growth in travel abroad and the globalization of higher education, many institutions' policies and practices do not require the development of globally competent perspectives for students. When universities globalize and internationalize, students need to be equipped with the skills to negotiate these aspects of higher education if they are going to be successful graduates. The classroom is one of the places that the global-self can be developed (Chang, 2006; Urban, 2012; Urban \& Palmer, 2014), but activities outside the classroom, where co-curricular learning can be activated, also impact student development (Astin, 1984; Killick, 2015; Kuh, 1993; Soria \& Troisi, 2013; Tierney, 1992; Tinto, 1975).

Co-curricular learning, learning that happens outside the classroom in support of university missions, visions, and values is an important component of the student experience. The interactions between international and domestic students impact both groups' development of their global-self. There are many environments for co-curricular learning, including areas such as service learning, student government, residence life, and campus recreation. Of these environments, this study is interested in students' engagement in sport in a collegiate recreation setting as a place where international and domestic students can interact, where intercultural competence can be developed, and where student development can be nurtured. 


\section{Intercultural Competence}

Understanding cultural difference, and having the attributes needed to successfully negotiate interactions between individuals from different cultures, is one description of intercultural competence. Almost any difference between two groups of people can be a cultural difference. For example, culture may be categorized by race, ethnicity, socioeconomic status, gender identity, or similar types of differences.

Having intercultural competence, and thus the ability to promote sustainable human development, relies on the interactions on campus between students who have different cultural backgrounds (Gacel-Ávila, 2005). How a student experiences internationalization - that is, how they draw upon their skills, knowledge, and personal attributes - is essential to improving students' understanding of the cultural differences between individuals (Johnson, Lenartowicz, \& Apud, 2006). For international students, developing intercultural competence is more readily achieved when they are in environments where they are provided freedom to create who they are to develop their self-formation (Marginson, 2014). Marginson (2014) points out that "international education as self-formation means that instead of the international student being seen as habitually weak or deficient, the student is understood as typically a strong agent piloting the course of [their] life" (p. 12). The accomplishment of this self-formation is learned over time through experiences and reflection and is important for both international and domestic students.

Higher education is often the final step in the formal education process, thus intercultural competence is critical for students to develop if they are going to have a global-self. One of the markers of intercultural competence skill development is the 
behavioral adaptations that individuals undertake to interact effectively with people from different cultures (Johnson et al., 2006). Sports are an environment conducive to observing, participating in, and creating behavioral adaptation. The next section will discuss how sport and leisure play a role in supporting behavioral adaptations that support intercultural competence.

\section{The Role of Sport and Leisure}

Sport is viewed as a universal connector. Regions of the world take pride in their abilities in sport and use this pride as one way to build cultural identity (Donahue, Rip, \& Vallerand, 2009). Sport has a large impact on humanity and often helps create positive social change. Consider that over 120 million people watched the 2015 Super Bowl and that over one billion people watched the 2014 World Cup soccer final. As a stage for social action, sport has had many defining moments: Jesse Owens winning in Hitler's Olympics, Tommie Smith and John Carlos' Black power salute, the horrific hostage taking in the Munich Olympics, Caitlin Jenner's transformation from Olympic decathlete champion to world transgender champion, and the events at the University of Missouri in 2015 where both the President and Chancellor-under pressure for their responses to minority students - were forced to resign in the face of a potential collegiate football player strike. The issues facing today's students involving race, religion, gender identity, sexual orientation, and nationality are intertwined with our culture's fascination with, and participation in, sport (Ladda, 2014). Sports can be an entry point for students discussing and engaging in conversations about these issues.

On university campuses, many students participate in sports through intramurals, McClellan, King, and Rockey (2012) define intramurals as 
school-based recreational sport pursuits involving some form of competition between one or more participants. The key element of intramural sport is the freedom of choice. Unlike intercollegiate athletics, the participants have a choice in programs in which to participate, the formats, and at what level to compete. Intramural programming is a staple for most campus recreation departments and may range from a fishing tournament to the more traditional flag football and basketball. (p. 30)

Sport plays an important role for many international and domestic students in their individual identity development and plays a role in the social recognition and engagement that is part of developing the global-self (Weiss, 2001). Sport can simultaneously help international students maintain their home-culture while also helping them adapt to a different environment (Allen, Drane, Byon, \& Mohn, 2010). Students who participate in intramural sports can improve self-confidence, increase understanding and tolerance of different cultures, decrease social alienation, improve their sense of belonging to the university, and increase trust in peers (Artinger et al., 2006). Sports can have a positive impact on a students' experience and help them develop attributes of the global-self, including a sense of belonging and understanding of different cultures.

\section{Engagement}

Co-curricular activities, such as intramural sports, play an important role in intercultural competence (Soria \& Troisi, 2013). Soria and Troisi (2013) reviewed student experiences with internationalization at-home activities at nine large public research universities and propose that "internationalization at-home activities can also promote students' development of global, international, and intercultural competencies just as effectively as - if not more effectively than —-formal study abroad" (p. 273). Halfway around the world, Jon (2013) found this same effect to be true for Korean domestic students. At-home can be defined in two ways. The term can define activities 
that provide an international context in a domestic setting, and the term is often used interchangeably with domestic to define a student who is from the place where an activity takes place. Both definitions will be used in this paper.

Soria and Troisi (2013) provide several ways the at-home activity can take place, including attending global or international focused lectures, workshops, or performances; enrolling in courses with an international theme; or participating in living-learning environments. These activities often promote social engagement between international and domestic students in classrooms and outside of classrooms (Hser, 2005). The cocurricular experiences where at-home activities often happen are in student affairs, the department or division of services and support for students that enhance student growth and development outside the classroom. The range of departments in a typical student affairs portfolio is broad and may include, but is not limited to student health, student unions, student legal services, student leadership, residence life and housing, dean of students, multicultural student services, admissions, orientation, and campus recreation.

Some international students shape their identity through their leisure or sport activities, and these activities are an important piece of their engagement on campus (Allen et al., 2010). Yet, as international students establish themselves within their new culture, they often run into a circular problem: They are often studying abroad to learn about a new culture, but spending time with students of their original culture has positive outcomes for reducing stress, and stress is a major factor in student's being unsuccessful in a university (Murff, 2005). International students will thus rely on fellow international students to help address acculturative stress - or the stress associated with adapting to a 
new culture - and not interact with domestic students (Smith \& Khawaja, 2011). Despite these stressors, most international students still want American students to try and get to know them and their culture (Urban \& Palmer, 2014).

Individual identity development is a core outcome of a successful university experience (Baxter Magolda, 2003) and improving the engagement between and among domestic and international students can help international students adjust to an American university and keep them engaged academically and socially. Unfortunately, because international students are often lumped together without regard for their unique cultural identities (Baxter Magolda, 2003), there are a number of barriers to successful engagement between international and domestic students.

Campus recreation is an example of a co-curricular program for studentssponsored by the university — that is designed to support and enhance student success. Most often housed in student affairs, campus recreation is defined as part of an institution's community life that provides a set of programs, activities, and physical environments for the members of the community to participate in fitness, wellness, and sport (McClellan et al., 2012). Campus recreation is unique from athletic programs in that it is open to all members of the community and not solely focused on competition; it is unique from health services in that its activities are almost always proactive in managing health. This study is linked to the internationalization of campus recreation in the United States and how it can support the development of intercultural competence and the creation of a global-self. 


\section{Summary}

Killick's (2015) strongest assertion is that for a student to develop a global-self, they need to know that others with whom they exist are equally human. While many students develop "a higher level of open-mindedness" (Twombly, Salisbury, Tumanut, \& Klute, 2012, p. 73) through their travel aboard experience, many students do not have the luxury to study abroad due to the high cost, minority group marginalization, or the fear of unfamiliar places (Soria \& Troisi, 2013). It is particularly important to create environments for students to engage in activities on campus that develop similar changes in their global-self as they would get traveling abroad. Universities are often responding to this idea by internationalizing their campuses because internationalization is beneficial to supporting students' intercultural competence.

Through engagement in activities outside of the formal curriculum, students can have interactions that help them develop their ability to act-in-the-world, which supports their concept of self-in-the-world. Studying how these interactions impact students, how they occur in campus recreation, and how students perceive they influence the development of their global-self through their participation in intramural sports is important for improving the collegiate recreation profession and the field of student affairs.

\section{Statement of the Research Problem}

Students can develop global, international, and intercultural competencies while staying at home on their domestic campus through their participation in global/international co-curricular activities (Soria \& Troisi, 2013). Yet, international students' learning and self-perception, important pieces of the global-self, can be 
negatively influenced by their time at U.S. institutions (Schmitt, Spears, \& Branscombe, 2003; Urban \& Orbe, 2007). Part of the challenge for higher education staff is to develop co-curricular experiences that improve the intercultural experience for international and domestic students when they interact. But simply recruiting international students to a campus and expecting that they will automatically interact with domestic students, thereby increasing everyone's intercultural skills, does not happen spontaneously. Intentionality in the design of co-curricular interactions is essential for effective intercultural development.

The co-curricular opportunities in collegiate recreation provide a useful site for these interactions, but professionals in collegiate recreation have little understanding of whether anything they do helps students to develop their global-self. There is a lack of research on collegiate recreation's impact on students' intercultural competence and a broader lack of research in addressing how universities create strategies to help students intentionally develop their global identity (Croom, 2014). In researching the development of the global-self, it is helpful to understand how students gain the skills and selfawareness to positively engage in the new global paradigm since students "perceive it is the responsibility of educational institutions to increase and enhance intercultural interactions" (Ward, 2001, para. 16). There are benefits to engaging in team sports, including opportunities to learn about the other, to identify cultural misunderstandings, and to work through conflict (Giulianotti, 2011; Höglund \& Sundberg, 2008; Kidd, 2008; Walseth \& Fasting, 2004). Intramurals is one way students can participate in team sports on campus. By researching how participants in intramurals perceive they develop 
intercultural competence, practitioners in recreation and other student affairs areas may be better able to design intentional programs to help students develop their global-self.

\section{Purpose of the Study}

While cultural fairs are plentiful, international students sit in classes, and glossy posters of travel abroad dot the hallways, international and domestic student interactions are often haphazard and random (Glass, Buss, \& Braskamp, 2013; Mazon, 2010; Smith \& Khawaja, 2011). The broad problem in higher education is that there are not enough structured and intentionally designed co-curricular opportunities in universities to create the situations where students interact to create their global-self. The purpose of this study is to examine students' perceived development of their global-self when they participate in an intentionally designed intramural soccer program.

Killick's (2015) belief that students can consciously work toward the development of the global-self by simply getting to know each other in and out of the classroom provides the framework for this study:

A further change in what the world presents or a change in how [the student] responds offers new learning opportunities. If this were not so, little of significance could happen at a university; if it is so, then universities do hold the potential to enable students to develop into more global selves. (p. 104)

By embracing Killick's belief that universities hold a special place to develop the globalself, the design of the study included teaching about a global perspective in student staff training and developing interventions to the typical participant experience to support the development of students' global-self, thereby improving the profession of collegiate recreation and student affairs. 


\section{Significance of the Research}

In U.S. history, there was little focus given to international education until Russia successfully launched Sputnik in 1957. Until that time, the United States enjoyed a dominant role in global politics for nearly 200 years, unimpeded by competing countries and mostly immune to global economic challenges. The response to Sputnik was fast and consistent. The United States first responded with the National Defense Education Act of 1958, then the International Education Act of 1966, and then the National Security Education Act of 1991. These policies created justifications for increasing our global knowledge through the study of global issues and helped lead to the large numbers of students studying abroad that we see today.

Unfortunately, the flow of students around the world studying abroad has not solved our propensity for conflict. In Fall 2015, hundreds of thousands of people were fleeing the Middle East and Africa for Europe, creating cultural conflicts and demonizing immigrants. Simultaneously, the politics of the United States became more divisive. Multiple U.S. presidential candidates expressed outrage at "illegal immigrants" and threatened to deport them all; one presidential candidate went so far as wanting to ban all Muslims from entering the country (Bermen, 2015). In Fall 2017, that candidate, Donald Trump, became President of the United States. Political solutions to these cultural conflicts tend to focus on short-term accommodations since long-term solutions are hard to find. This fear of immigrants has ripple effects that reach higher education institutions and impact the ability to effectively work with, and recruit, international students (Charles-Toussaint \& Crowson, 2010). Having international students on campus is essential if we hope to use education to counter the false narrative of immigrants, or 
anybody not domestic, as dangerous and unequal. If higher education is going to develop citizens who care about the security of the world, the development of the global-self is an important aspect of building this narrative.

In the development of the global-self, cultivating the ability to see that each person has an equal right to be human is a critical component (Braskamp, 2008; Caruana \& Ploner, 2010; Killick, 2015). In a time when conflicts are simmering and solutions to global violence seem distant, the importance of education to creating systemic change is essential. When there is a chance to have intentional dialogue with a person of difference, our compassion can grow and the development of the global-self can occur.

Sport plays a role in supporting racial and ethnic identity development in higher education, which in turn helps promote cultural understanding (Beamon, 2014; Karkouti, 2015; Pieterse, Carter, Evans, \& Walter, 2010). The development of racial and ethnic identity in higher education is important, but research on the nexus of international students, sports, and the development of racial and ethnic identity is limited. Students who can find their identity on campus are ultimately more likely to stay on campus and graduate (Chickering \& Reisser, 1993; Marginson, 2014; Tinto, 1975), improving the probability of creating a more educated citizenry.

Collegiate recreation engages over $75 \%$ of students in the United States (Forrester, 2014, p. 6) and professionals in collegiate recreation are looking for ways to help international and domestic students learn from each other. NIRSA: Leaders in Collegiate Recreation (NIRSA, 2015), the leading professional development organization for collegiate recreation, has identified a Global Perspective as one of its core values. Students "perceive it is the responsibility of educational institutions to increase and 
enhance intercultural interactions" (Ward, 2001, para 16), and by researching how sports changes students' perception of their intercultural competence, practitioners in recreation or other student affairs areas may be able to learn how to design intentional programs to help students reflect on their own global-self development.

This study will contribute to professional knowledge for the collegiate recreation professional and provide transferable ideas and methods about how to support developing student's global-self. This study is applicable across the recreational sports profession, whether in higher education, community recreation, or even youth recreation. Sport, and soccer specifically, are global in nature, making it possible for this study to be replicated in multiple environments. While the role sport plays on campus may be different across regional models of higher education, the development of intercultural competence of international and domestic students because of their joint participating in sports is worth exploring.

\section{Research Methodology and Research Questions}

The development of the global-self relies on students to work toward an esoteric goal of self-awareness, and according to Killick (2015), it relies on the assertion "that the others with whom I coexist are equally human" (p. 31). To assess a student's progress toward global-self is difficult as there are many variables that impact self-awareness and skill development (Deardorff, 2006). Deardorff's (2006) review of best assessment practices recommends assessment that is "primarily qualitative in nature, including the use of interviews, observation, and case studies, as well as the possible use of standardized competency instruments" (p. 258). Using this advice as a guiding principle, 
this dissertation used an 8-week case study of an intramural soccer program to answer the following two research questions:

- How do students participating in a collegiate intramural soccer program perceive the development of their global-self after experiencing interventions intentionally designed to enhance discussion of, and reflection on, attributes of the global-self?

- How do students experience an intramural soccer program designed to encourage the development of global-self characteristics?

To help answer the question, I drew from community action research to design intentional interventions that support the development of the global-self in the students and staff who participate. My study began with a document review of the current staff training materials to understand the prior training of student staff and then, drawing from community action research, I trained the student staff on intercultural competence and the global-self. Together with the student staff, I developed a team captain's training and inseason interventions designed to improve students' global-self. I observed the interventions and took field notes to capture my observations. I used two forms of assessment to understand the student experience. I used an open-ended survey to understand students' experience of the interventions, and then I conducted seven semistructured interviews. A professional colleague conducted an additional two interviews of student staff. The observations, surveys, and interviews helped me to draw conclusions and make recommendations for improving the way that professionals in collegiate recreation train their staff, implement their programs, and educate participants about the global-self. 


\section{Summary and Overview}

Nearly 20 years ago, Dalton (1999) argued that a new mindset was needed for student affairs professionals_-like those in collegiate recreation-if they were to be effective in supporting internationalization: "[I]t is especially important that student affairs professionals not allow themselves nor their profession to be further marginalized by lack of knowledge and experience in the international dimensions of higher education leadership" (p. 4). Dalton's advice is still relevant. The need for higher education professionals to understand their role in helping develop a global perspective is paramount with the ever-growing globalization and internationalization of universities. Killick's (2015) admonishment that "a university education will only effectively contribute to our students' development of global selves if we attend to all aspects of the complexity which constitutes their lived experience and give shape to who they are" (p. 179) helped sculpt the form of this study. This study used a case study of an intramural soccer program that had intentionally designed interventions, developed using aspects of community action research, to encourage students to reflect on aspects of their globalself. Observations, surveys, and interviews assisted in the assessment of the interventions.

Chapter 2 of this dissertation provides a literature review of the major components of this study, including research and theoretical aspects related to developing the globalself through sports in collegiate recreation environments. Chapter 3 outlines the methodology used to complete the research, including a description of the research perspective and a review of the procedures used during the research interventions. Chapter 4 presents the results of the study in two sections; the data are presented as themes correlated with aspects of the global-self and are presented through evaluative 
data demonstrating how students experienced the interventions and their impact on the quality of the intramural program in which they participated. Chapter 5 includes a discussion of the study findings, their implications for collegiate recreation and student affairs practitioners, and potential areas for future research.

\section{Definitions}

Internationalization. Internationalization includes the range of activities, policies, and practices universities use to improve intercultural interactions between students, between students and staff, and between students and faculty who identify as being from different countries or global regions.

Globalization. Globalization refers to the strategies and initiatives higher education institutions use, including activities designed to engage across international borders, to produce communities who embrace the importance of internationalization.

Intercultural competence. Intercultural competence is understanding cultural difference and having the attributes needed to successfully negotiate interaction between individuals from different cultures.

Global-self. Global-self has two components: the ability to understand your selfin-the-world (primarily the acceptance that the others with whom a person coexists are equally human), and second, the ability to act-in-the-word, or "the knowledge, skills, and values which enable [the student] ... to take deliberative action with appropriate references to the contexts and participants implicated in the action" (Killick, 2015, p. 31).

Campus recreation. Campus recreation is part of an institution's community life that provides a set of programs, activities, and physical environments for the members of 
the community to participate in fitness, wellness, and sport. It is distinct from varsity athletics or physical education.

Intramurals. Intramurals are "school-based recreational sport pursuits involving some form of competition between one or more participants" (McClellan et al., 2012, p. 30).

Student affairs. Student affairs is a term that refers to the department or division in a higher education institution that provides services and support for students that enhance student growth and development outside the classroom.

At-home. The term at-home refers to activities in an educational setting that provide an international context in a domestic setting; the term is used interchangeably with domestic to define a student who is from the place where an activity takes place. 


\section{CHAPTER 2: LITERATURE REVIEW}

This chapter contains four sections reviewing the literature related to the development of the global-self and its relationship to higher education and collegiate recreation. First, a thorough review of Killick's (2015) notion of the global-self will provide a deeper understanding of his theory, its relationship to the literature, and its applicability. The next topic reviewed is how institutional perspectives on the curriculum and the environment provide a framework supporting, or opposing, efforts to include a global perspective on campus. Then, student engagement, intercultural development, and learning theories are reviewed to shape the rationale for the type of study proposed. Finally, sport and recreation for social change literature is reviewed to place this study in the context of sports and to highlight the potential for sport and recreation to influence the development of the global-self. A summary of the chapter sets the stage for the discussion of the methodology in Chapter 3.

The literature on international students and internationalization spans multiple countries and various models of higher education. Australian, Canadian, British, and American scholars have produced much of the research on international students and on the internationalization of universities. Because the studies draw on each other, and because of the implicit understanding that we operate in a global world, minimal reference is made to the home country of the research. In general, much of the literature is based on Western ideas and research; it is noted when the literature is based in a nonWestern context.

\section{Killick's Global-Self Theory}

Killick's (2015) concept of the global-self is an expansion and a critique of the 
more commonly used term global-citizen (Braskamp, 2008). An understanding of Killick's concepts and theories about the global-self connect the literature review to the focus of the study. Killick describes two attributes students need if they are to successfully develop their global-self: self-in-the-world and act-in-the-world.

First, Killick (2015) described a state of awareness that he defined as "self-in-theworld, namely, that the others with whom I coexist are equally human" (p. 31). For Killick, self-awareness is not necessarily about liking the "other," nor is it something accomplished easily; it also does not require agreement with another person. Acknowledging that others are equally human does require, however, that "global students and global selves need an equally skeptical stance towards their own ways of being as they have towards those of others" (Killick, 2015, p. 31). Killick argued that this skeptical look at ourselves - at our assumptions and prejudices about others - is why internationalization of higher education is vital for today's students. Without internationalization, challenging our assumptions or developing the skills necessary to act-in-the-world — the second attribute of Killick's global-self—is more difficult.

This second attribute - the ability to act-in-the-world — requires the "knowledge, skills, and values which enable [the student] to act-in-the-world; by which [students have] the capabilities to take deliberative action with appropriate references to the contexts and participants implicated in the action" (Killick, 2015, p. 31). This ability to act-in-the-world is distinct from the awareness of self-in-the-world; each of the two aspects of the global-self can operate independently. A student may have self-awareness of their place in the world and may believe they are equally human with others, but they may not have any tools to put this awareness to work. Conversely, a student may have the 
skills to act-in-the-world, proficiency of a second language or cultural practices for instance, but not have the self-awareness to treat the other as equally human. Both aspects of the students' development need to occur, and overlap, for the global-self to come to fruition.

The overlap of self-in-the-world and act-in-the-world is essential to Killick's (2015) premise that

to make their way in the increasingly interconnected and diverse globalizing world, and to do so in ways that are commensurate within a broad agenda of social justice, our students need opportunities to experience being global students in order to develop their capabilities as global selves. (p. 32)

It is important to note that being and doing are linked in Killick's formulation of the global-self. To apply Killick's theory to the problem-in-practice, being and doing will both be analyzed in the literature as they relate to institutional perspectives, student engagement, intercultural competence formation, learning, and the context of sport and recreation for social change.

\section{Institutional Perspective}

Higher education around the world has responded differently to the internationalizing student body. A meta-review of literature found that students "perceive it is the responsibility of educational institutions to increase and enhance intercultural interactions" (Ward, 2001, para. 13), yet “"internationalization’ remains for many an income generation exercise" (Killick, 2013, p. 721). Killick (2015) breaks down the potential institutional response to internationalization into four different areas: (a) shaping the environment, (b) shaping the curriculum, (c) shaping the delivery, and (d) 
shaping broader institutional practice. While each of these aspects is important to the development of the global-self, this section will focus on the attention given to shaping the curricula and shaping the environment.

\section{Shaping the Environment}

Institutional practices such as curriculum design are most often associated with student learning; time in the classroom clearly impacts students understanding of diversity and intercultural competence (Chang, 2006; Gurin, Dey, Hurtado, \& Gurin, 2002). However, the environment outside of the classroom is also essential to learning about the global-self. Braskamp (2008) argued that "we need to design better environments that integrate learning and development, that is, we need to think and act from a holistic and integrative perspective" (p. 4), and he challenged "the academy ... to provide the experiences that integrate how students think, feel, and relate to others" (p. 4). Environments can be meta-environments (e.g., what the general feel is on campus based on attributes like university type, location, or morale) or micro-environments (e.g., what is in the student-union building or how a recreation center lays out equipment).

The shaping of the university environment starts with the mission and vision, and while mission and vision statements are often confused, both are used as a base for decision-making, serve as beacons for direction, and directly influence organizational environment (Raynor, 1998). Creating an environment that recognizes the importance of internationalization, and its impact on the development of the global-self, has not historically been a part of these guiding documents, but the tide may be turning as "internationalization has become more widely embraced in higher education institutions around the world, and its importance can be seen by its increased presence in institutional 
mission statements" (Hendrickson, Lane, Harris, \& Dorman, 2013, p. 63). It is the mission — partnered with policies and strategic plans — that leads to successful internationalization initiatives (Knight, 2004). Tierney (1988) encouraged the examination of mission and vision to understand what is important to the university's culture. With no clear vision for internationalization, it is difficult to create integrative efforts that create a change to university culture (Glass et al., 2013). Comparing four university vision statements demonstrated the different views that universities have toward internationalization and the subsequent outcomes that emerge.

Northeastern University's (2015) vision statement is a strong example of clearly prioritizing internationalization: "Our vision is to be a university that expands the meaning and impact of our engagement in the world using our knowledge and resources as positive forces for change in both our local communities and our global society" (para. 2). The vision statements of schools in Oregon are not as directive: Portland State University (2016) envisions that it "leads the way to an equitable and sustainable future through academic excellence, urban engagement, and expanding opportunity for all” (para. 1); Oregon State University (2015) “promotes economic, social, cultural and environmental progress for the people of Oregon, the nation, and the world" (para. 4); and the University of Oregon's (2015) vision is, among other things, to “enrich the human condition" (para. 3). On one hand, each of these statements acknowledge the challenges of equality and diversity, on the other hand, none of the Oregon schools explicitly acknowledge the "intersection between internationalisation, equality and diversity, and inclusivity 'at home"' (Killick, 2013, p. 221). This does not mean the Oregon universities are not trying to strengthen their internationalization, but it might 
explain why Northeastern has more than double the number of international students attending than its nearest competitor in Oregon (IIE, 2014), undoubtedly creating a different environment that is more conducive to developing global perspectives.

Because of the difficulties that come from not having a mission or vision with clear links to internationalization and globalization, Coryell, Durodoye, Wright, Pate, and Nguyen (2012) suggested that since

there is no one way to implement international education across the institution ... there is a need for the entire university community (faculty, students, administration, and staff) to develop and acquire intercommunication skills, knowledge of international practices in one's discipline, and transcultural sensitivity. (p. 16)

To accomplish this goal, they advocated the development of "a new mind-set" that is "supported [through] the professional development of faculty, administrators, and staff" (Coryell et al., 2012, p. 18). While a university may or may not provide a mission or vision directly related to internationalizing a campus or creating global students, there are still important institutional practices that make progress toward these goals, including how we use the environment to form community and engage students in their learning.

Community takes many forms. Himanka (2012) referred to Snellman's definition of the university as a community of selves where students exist together to learn "to think from the point of view of another self within the community" (p. 527), while MacQueen et al. (2001) had a more practical definition of community as "a group of people with diverse characteristics who are linked by social ties, share common perspectives, and engage in joint action in geographical locations or settings." (p. 1,936). The role of community in learning about the self-in-world is vital. Vygotsky's (1978) zone of proximal development required people to be in community with others for learning to 
happen, and Killick (2015) suggested that community can result in spaces where "coparticipants, perhaps as more knowing others, perhaps as peers, perhaps as dwellers in a similar world, but perhaps more powerfully as different others, help shape all the learning" (p. 118). The learning environments on campus can have a significant impact on the development of communities and the subsequent learning that occurs in those communities.

\section{Shaping the Curriculum}

The formal curriculum can consist of the "sequenced programme of teaching and learning activities and experiences organized around defined content areas, topics, and resources, the objectives of which are assessed in various ways including examinations and various types of assignments, laboratory sessions, and other practical activities" (Leask, 2009, p. 206). The curriculum is often designed by faculty and approved by faculty senates, and it is often organized through curriculum designed to reinforce a particular perspective: a Christian university may have academic course requirements about Christian teachings, while agriculture schools may have more majors and classes on agriculture. The curriculum creates the type of student that a university wants to graduate, and if international education is "an important educational mega-goal that should permeate the entire educational system" (Mestenhauser, 1998, p. 4), it is imperative for an institution to examine its role in internationalizing the curriculum. Internationalizing the curriculum is "the incorporation of an international and intercultural dimension into the content of the curriculum as well as the teaching and learning arrangements and support services of a program of study" (Leask, 2009, p. 209). Universities in the United States appear to be behind their peers in shaping an 
internationalized curriculum. Leask (2013) wrote that "most Australian universities now have statements of graduate qualities or attributes focused on the development of global perspectives, cross-cultural communication skills, intercultural competence, world knowledge, and so forth" (p. 105), while initiatives coming out of Great Britain focus on combining "an institution-wide initiative delivered with the disciplines" (Jones \& Killick, 2013, p. 166). Hser (2005) noted that "in comparison with other advanced industrial nations, the U.S. lags behind in realizing the growing interdependence of the world" (p. 38) and that "American professors were the least involved in international activities such as studying and doing research abroad" (p. 38). There is work to be done in American higher education if we are to help our students understand their role in the globalizing world.

Because many universities lack curriculum or a mission that addresses internationalization and globalization, and due to the increase in student mobility around the world,

there is a growing awareness that internationalising the curriculum should not be conducted simply to account for an increasing number of international students in the classroom. It should also be appreciated as an institution's self-awareness to prepare its students to be global citizens who can operate in a globalised world. (Schuerholz-Lehr, as cited in Sawir, 2013, p. 361)

Building on this belief, Killick (2015) appealed for a major reconceptualization of the university, advocating for a "university experience which focuses primarily on establishing global identity/self-realization" (p. 126). Motivations for internationalizing the curriculum should, therefore, not only focus on international students, but on the development of students who can be active and critical citizens (Leask, 2005). 
In two forms of the curricula, the hidden and the informal, there are similar concerns about how internationalization is missing or misunderstood. The hidden curriculum includes the messages - explicit and implied — that are woven into the community and describe an institution's values. These messages are delivered through such things as policies, athletic mascots, marketing logos, building design, and invited speakers (Crowther et al., 2000; Killick, 2015; Snyder, 1971). The informal curricula are the programs and activities that happen on and around the university throughout the day, sometimes provided by the university and sometimes not (Killick, 2012, 2015; Leask, 2009). Examples range from the conversations students have with each other or what one might overhear in line for coffee, to more structured activities that occur in co-curricular opportunities such as residential housing or student activities or campus recreation. While "curriculum" is usually associated with what is taught in the classroom, there is an emerging recognition that the hidden and informal curricula can impact internationalization efforts and development of the global-self. Leask's (2009) definition of the internationalized curriculum recognized "the need to create a campus culture of internationalization, one that encourages and rewards intercultural interaction both outside and inside the classroom" (p. 106).

Interactions outside the classroom are part of the hidden and informal curricula where learning also happens and gets interwoven in the campus community (Killick, 2015). Important concepts like the global-self can be embedded into these hidden and informal curricula to create experiences and environments to facilitate learning (Parsons, 2009). The shaping of the hidden and informal curricula sends messages about the importance of internationalization and need to be acknowledged when intentionally 
designing co-curricular opportunities. The environment, often molded by the mission and vision, combines with the curriculum to create the type of student that a university graduates. Each piece of this institutional perspective is essential to the development of the global-self.

\section{Student Engagement}

Student engagement is related to involvement (Astin, 1984a), persistence (Tinto, 1975), growth (Chickering \& Reisser, 1993), and the out-of-classroom experience (Kuh, 1995). Astin (1984), Tinto (1975), Chickering and Reisser (1993), and Kuh (1995) each researched different aspects of student engagement, and collectively their research endorsed student engagement as a vital aspect of student success. Student success includes important outcomes like academic grades, retention rates, satisfaction, and graduation rates, but it also includes the development of the whole student. Student engagement, essential to developing the ability to act-in-the-world, is defined by the National Survey of Student Engagement (NSSE, 2016) and

represents two critical features of collegiate quality. The first is the amount of time and effort students put into their studies and other educationally purposeful activities. The second is how the institution deploys its resources and organizes the curriculum and other learning opportunities to get students to participate in activities that decades of research studies show are linked to student learning. (para. 1)

This research reviews foundational literature on involvement, integration, personal growth, and engagement and draws connections on how engagement helps students develop their global-self. 


\section{Astin and Student Development}

Many of today's student engagement ideas find their roots in Astin's (1975) theory of student involvement. Astin (1999) defined an involved student as "one who ... devotes considerable energy to studying, spends much time on campus, participates actively in student organizations, and interacts frequently with faculty members and other students" (p. 518), and he argued that involvement is key to student success. The depth and quality of student involvement relates proportionally to potential student learning and development. Astin's (1999) research argued that students improve their chances in college by being more involved and, importantly, that universities have within them the ability to do something to encourage this involvement.

Astin's (1984) argument on involvement relies on three implied theories grounded in the academic pedagogy: (a) subject-matter theory, which relies on the exposure to the right subject matter (often related to curricular development); (b) resource theory, which relies on ensuring the right number of resources (e.g., studentfaculty ratio, physical facilities, fiscal resources); and (c) individualized theory, which suggests that an individualized approach to learning is needed (e.g., electives, guidance counselors, self-paced instruction).

In his student involvement theory, Astin (1984) linked these three theories together, acknowledging that student involvement is not linear, does not happen in isolation, and that each part of the university can play a role in encouraging involvement; every time a student engages with an aspect of campus provides an opportunity for deeper involvement. However, because students have limited time in the classroom to learn, Astin's theory argued that it is important to maximize non-classroom time to 
supplement formal learning. Astin (1999) noted that "simply by eating, sleeping, and spending their waking hours on the college campus, residential students have a better chance than do commuter students of developing a strong identification and attachment to undergraduate life" (p. 523). The idea that all time is potentially learning time has greatly shaped how student affairs professionals implement co-curricular student development.

Building on Astin's concept that all time can be learning time, M. Johnson's (2014) study analyzed data from the Multi-Institutional Study of Leadership to examine the predictors that led students to engage in social change behavior. M. Johnson (2014) found that

collegiate experiences such as involvement in a student organization (either as a general member or formal leadership position), leadership training and education, and discussing major social issues were shown to be the largest predictors of students engaging in social change behavior. (p. 155)

Astin's (1984) involvement theory is a major contributor to the application of cocurricular learning in the collegiate recreation context (Artinger et al., 2006; Busby, 2011; Guo \& Ross, 2014; Kim, 2012). The importance of the leadership training, education, and discussion of major social issues as part of that involvement are essential to this study.

\section{Tinto and Integration}

Concurrent with Astin's (1975) early work on involvement was Tinto's (1975) foundational work on why a student might dropout. Tinto asserted that academic difficulties were not the only reason students dropped out. Tinto proposed that a lack of social integration plays an important role in student persistence and that intentional actions could be taken to improve student integration. Using Durkheim's (1961) suicide 
theory that people commit suicide when they are "insufficiently integrated into the fabric of society" (p. 91), Tinto argued that if universities are their own mini-societies, with their own value and social structures, then it would make sense that people would dropout for similar reasons of non-integration. Tinto (1975) suggested that "other things being equal, the higher the degree of integration of the individual into the college systems, the greater will be his commitment to the specific institution and to the goal of college completion" (p. 96). Like Astin's involvement theory, Tinto’s (1975) definition of social integration included "informal peer group associations, semi-formal extracurricular activities, and interaction with faculty and administrative personnel within the college" (p. 107). It is not just the act of social integration, but the perception of social integration that matters to students' persistence. In other words, unlike Astin's proportional relationship between time and engagement, Tinto argued that regardless of their actual time engaged, if students felt engaged, they were more likely to stay in school.

With this in mind, persistence is influenced by the choices students make between engaging in activities that relate to their education or engaging in activities that do not relate to their education (Tinto, 1975). Tinto's conclusions have driven professionals to be strategic about facilitating student integration on campus (Sawir, Marginson, Deumert, Nyland, \& Ramia, 2008; Söderqvist, 2007; Tierney, 1992). Killick (2015) related integration to the ability of a student to act-in-the-world, noting "if our students feel uncomfortable in their interactions with others, how then will they find the capability to engage in respectful dialogue or to seek out how the world looks from other's perspective?" (p. 108). Using sports to help students feel integrated is one tool 
universities are using to help students move through their development phases, which is discussed in the next section.

\section{Chickering and Reisser}

While Astin $(1975,1984,1999)$ championed involvement and Tinto (1975) advocated for institutional integration, Chickering and Reisser's (1993) developmental model described the student development that occurred as a result of student engagement. Using seven vectors - developing competence, managing emotions, moving through autonomy toward interdependence, developing mature interpersonal relationships, establishing identity, developing purpose, and developing integrity-Chickering and Reisser's model proposed that students move through the vectors in different ways, often progressing along one vector only to realize that more work needs to happen in a vector they already visited. The model allowed for students to be moving along multiple vectors at the same time as they work toward establishing their identity.

The model described individual identity development occurring as students come across new ideas from the formal and informal curricula. Student development along the vectors supports "communion with other individuals and groups, including the larger national and global society [emphasis added]" (Chickering \& Reisser, 1993, p. 35). The fourth vector - developing mature interpersonal relationships — is especially relevant to students" movement toward a global-self as it describes a place where "friendships and student communities . . become meaningful subcultures marked by diversity in attitudes and backgrounds and by significant interpersonal exchanges" (Chickering \& Reisser, 1993, p. 48). The model placed “developing mature interpersonal relationships" before “establishing identity,” acknowledging "the importance of students' experience with 
relationships in the formation of their core sense of self' (Chickering \& Reisser, 1993, p. 39). The concept that students need to interact with each other is an cooperative process similar to Killick's (2015) global-self, where self-in-the-world and act-in-the-world are "intimately interwoven in our lifeworlds, and need also therefore to be intimately interwoven in the experiences which internationalization creates for the global student" (p. 32).

Chickering and Braskamp (2009) took special interest in how the vectors helped students "develop and internalize a global perspective into [their] thinking, sense of identity, and relationships with others" (p. 27). The four vectors they identified as most important included: (a) moving through autonomy toward interdependence, (b) establishing identity, (c) developing purpose, and (d) managing emotions (p. 27). The strategies used to accomplish the development in these vectors are developed in Chickering and Braskamp's (2009) Global Perspective Inventory, a tool measuring a student's global perspective:

First, a campus-wide initiative is required, with faculty as the key interventionists in creating in- and out-of-classroom conditions that foster a global perspective. Neither formal classroom instruction nor experiences such as travel abroad, community involvement, and social encounters are sufficient alone to guide students in their journey to become global citizens. . . . Second, bring the cultural differences associated with studying abroad into the campus culture. . . Third, use pedagogical strategies, based on active learning practices such as internships, field trips, and service-learning activities. Integrate these experiences and reflection with rigorous and critical, constructive, and creative thinking. (p. 30)

The four vectors laid out a path to investigate and support students' development of the global-self through engagement. 


\section{Kuh and Engagement}

Kuh's (1993) engagement model helps understand how engagement impacts the development of the global-self. The role of student involvement and development has informed how student engagement, "the time and effort students devote to activities that are empirically linked to desired outcomes of college and what institutions do to induce students to participate in these activities" (Kuh, 2009, p. 683) impacts student success. Building on the work of Astin (1975, 1984, 1999), Tinto (1975), and others, Kuh (2001) helped develop the NSSE. Student engagement, in large part due to this survey, has itself become an area of study that is worthy of its own literature review (Trowler, 2010). That review is beyond the scope of this study; however, there are multiple findings from Kuh that inform this study.

Through his initial interviews of 149 students, Kuh (1995) identified eight types of out-of-the-classroom experiences that were instrumental to learning and personal development: (a) specific leadership responsibilities, (b) interactions with peers, (c) academic-related activities, (d) “other" antecedents, (e) institutional ethos, (f) faculty, (h) work, and (i) travel. These out-of-the-class experiences, which include involvement in activities like student government, living in residence halls, and participation in campus recreation, have demonstrated positive outcomes on student engagement (Artinger et al., 2006; Astin, 1999; Seifert, 2014). Through these out-of-classroom experiences students' gains in interpersonal competence (self-awareness, social competence, self-esteem, and autonomy) were most associated with peer interactions (Kuh, 1995). Interacting with peers is also essential to Killick's (2015) advocacy for “establishing opportunities in classroom and campus spaces for a student to experience himself in successful 
interactions with diverse others, and to build such lived-experience into his biography" (p. 72).

The NSSE survey results inform many best practices for successful student engagement. For example, Kuh (2009) noted that "employment [on campus] may provide opportunities for students to practice and become more competent in collaboration and teamwork, skills that are needed to function effectively in the twenty-first century work environment" (p. 694). McEwan and Beauchamp (2014) defined teamwork as "a dynamic process involving a collaborative effort by team members to effectively carry out the independent and interdependent behaviors that are required to maximize a team's likelihood of achieving its purposes" (p. 233). Other terms such as collaboration or cooperation are used interchangeably with teamwork (McClellan, 2016) and help to encapsulate the types of skills needed for today's student to be able to act-in-the-world. Collaboration and teamwork are two aspects of Killick's (2015) tools for students to be able to act-in-the-world and are attributes often associated with participation in, and working for, collegiate recreation.

\section{Criticisms of Student Engagement Theory}

A point often overlooked in the research used to measure student development, involvement, and engagement is that the research did not respond in a timely manner to the "connection between culture, power, and knowledge" (Tanaka, 2002, p. 263).

Tanaka's (2002) investigation of higher education research methods critically reviewed the framework for these foundational pieces. Taking aim at Tinto's (1975) theory and methods, Tanaka questioned how factors related to student persistence could be measured if there were no questions about the impact of cultural history or racial shifts on student 
perceptions. Tanaka (2002) argued that Tinto's work on persistence is hard to validate since it is "unable to provide for an examination or issues of voice, power, authenticity, and reflexivity" (p. 273). Similarly, Tierney (1992) questioned Tinto's model since the model "never questions who is to be integrated and how it is to be done [and it] assumes an individualist stance of human nature and rejects differences based on categories such as class, race, and gender" (p. 611). Conversely, Tanaka applauded Astin's inclusion of critical questions related to gender and race in the Cooperative Institutional Research Program and implored Astin to explore "the impact of the college experience on the identities of white students as well as students of color and make possible an examination of interaction effects between institutional practices and the identity and power, on campus, of all students" (p. 275). The examination of power and voice moved the conversation to a different level and brought to the forefront the importance of intercultural development in conversations about the development of the global-self.

The foundational work of Astin (1975, 1984), Tinto (1975), Chickering and Reisser (1993), and Kuh (1995) moved the discussion of student success beyond historical measures of GPA and graduation rates to include the important aspects of student development. They successfully pushed for a broader definition of student development — one including students' lives outside of the classroom — and promoted the idea that higher education can be more successful when a holistic approach is used when working with students. The collective work around student development and engagement paved the way for a paradigm shift toward intercultural student development. The next section will examine intercultural development's role in our understanding of how student engagement can develop the global-self. 


\section{Intercultural Development}

The markers of intercultural maturity, an indicator that a student has developed their global-self, are defined in King and Baxter Magolda's model of intercultural development (Baxter Magolda, 2003; King \& Baxter Magolda, 2005; Perez, Shim, King, \& Baxter Magolda, 2015). King and Baxter Magolda's (2005) model originally drew on Kegan's (1994) model of lifespan development, defining the three forms of intercultural skills required for the mature level of development as a "complex understanding of cultural differences (cognitive dimension), capacity to accept and not feel threatened by cultural differences (intrapersonal dimension) and capacity to function interdependently with diverse others (interpersonal dimension)" (p. 574). The model took the three domains of development — cognitive, intrapersonal, and interpersonal — and defined the stages students can achieve in these domains as initial, intermediate, and mature. The model included transitional phases between levels (Perez et al., 2015). While students may bring different backgrounds to their initial placement on the scale, the goal of intercultural development for Perez et al. (2015) was for students to reach the mature level, characterized by the following attributes in Table 1.

The mature attributes of intercultural competence occur in Killick's (2015) attributes of the global-self as well. Self-in-the-world is found in the intrapersonal dimension where students focus “on how one understands one's own beliefs, values, and sense of self and uses these to guide choices and behaviors" (p. 574), while act-in-theworld attributes are found in the interpersonal dimension, which "focuses on how one views oneself in relationship to and with other people (their views, values, behaviors, etc.) and makes choices in social situations [emphasis added]" (p. 574) and in the 
cognitive domain by developing the ability to shift perspective to view issues through different cultural frames.

Gacel-Ávila (2005) advocated for a paradigmatic shift in students' development

of intercultural competence advocating that:

One of the basic and fundamental functions of a university should then be the fostering of a global consciousness among students, to make them understand the relation of interdependence between peoples and societies, to develop in students an understanding of their own and other cultures and respect for pluralism. ( $p$ 123)

International perspectives are crucial to the full intercultural development of students and universities should be responsible "for training citizens with a critical perspective and the

Table 1

Mature Level Attributes of Intercultural Development

\begin{tabular}{|c|c|c|}
\hline Cognitive & Intrapersonal & Interpersonal \\
\hline $\begin{array}{l}\text { Is able to consciously } \\
\text { shift perspectives and } \\
\text { behaviors into an } \\
\text { alternative cultural } \\
\text { worldview }\end{array}$ & $\begin{array}{l}\text { Considers collective } \\
\text { identities (e.g., Pan- } \\
\text { Asian) and interest in } \\
\text { coalition building }\end{array}$ & $\begin{array}{l}\text { Shows capacity to engage in } \\
\text { meaningful, interdependent } \\
\text { relationships with diverse others that } \\
\text { are grounded in an understanding of } \\
\text { and appreciation for human }\end{array}$ \\
\hline \multirow{5}{*}{$\begin{array}{l}\text { Is able to use multiple } \\
\text { cultural frames }\end{array}$} & Shows capacity to create & differences \\
\hline & $\begin{array}{l}\text { openly engages } \\
\text { challenges to one's views } \\
\text { and beliefs and that }\end{array}$ & $\begin{array}{l}\text { Understands how individual and } \\
\text { community practices affect social } \\
\text { systems }\end{array}$ \\
\hline & $\begin{array}{l}\text { (race, class, gender, etc.) } \\
\text { in a global and national } \\
\text { context }\end{array}$ & $\begin{array}{l}\text { Is willing to work for the rights of } \\
\text { others }\end{array}$ \\
\hline & $\begin{array}{l}\text { Integrates aspects of self } \\
\text { into one's identity }\end{array}$ & $\begin{array}{l}\text { Working for others' rights is } \\
\text { grounded in motivation to promote } \\
\text { social justice }\end{array}$ \\
\hline & $\begin{array}{l}\text { Displays integrated social } \\
\text { identities and understands } \\
\text { that multiple social } \\
\text { identities are part of self }\end{array}$ & $\begin{array}{l}\text { Builds relationships because of } \\
\text { differences rather than in spite of } \\
\text { them }\end{array}$ \\
\hline
\end{tabular}


and others

Shows appreciation for diversity

Sees the value of interdependent

relationships across differences

May negatively judge those who are perceived as somewhat narrow-

minded or culturally insensitive

Understands society as an organized entity that shapes social interactions and creates social inequalities

adequate preparation to work and live effectively and successfully in a global context"

(Gacel-Ávila, 2005, p. 125). One important outcome of developing this intercultural competence is the reduction of stereotyping. Killick (2015) challenged practitioners to be wary of using the identify of "international student" as a catch-phrase, instead encouraging the creation of

an inclusive environment [where there is] space for airing, sharing, and critiquing diverse perspectives; this must include building awareness and acceptance for gays, women, Muslims, and Chinese or South Korean students also hold diverse perspectives, and have a right to recognition and a voice as individuals. (p. 133)

To better understand how intercultural competence gets played out in practice on campus, Tierney (1992), as part of a 2-year study of Native Americans' college going behaviors, deconstructed the interviews of two college administrators. He found, much like Killick argued about internationals students, that the administrators believed student development theory had unintentionally created a view that cultural minority students (whether they be queer, international, female, minority status) have "problems" that somehow need to be "fixed." Tierney noted that universities mainly teach and do research from a Eurocentric, Anglo viewpoint where students of color or international students are 
viewed as deficient. Tierney (1992) advised practitioners to use theoretical frameworks, such as critical and feminist frameworks, "to consider how institutionally sponsored interventions function within the variety of different contexts that exist for different issues such as minority student retention" (p. 616). Killick (2015) similarly warned that “how we organize our students' experience of their university life may have some impact upon their coping strategies, attitudes, and proactivity, not least if we are able to enhance their experiences of intercultural contact" (p. 55).

Student interactions with institutions and individuals impacts their intercultural development, but "cultural diversity and internationalisation do not automatically lead to intercultural contacts and intercultural learning experiences” (Otten, 2003, p. 14).

Intercultural development can be successful when it has "reflection of individual and collective social experiences with people from other cultures rather than the mere contact as such" (p. 15). Saenz, Ngai, and Hurtado (2007), in their examination of factors that positively influenced interactions across racial categories, similarly encouraged directing that energy towards improving the cross-cultural interactions between students when they found

that students may not necessarily be engaging diverse others in more formal academic environments, but may be choosing to do so through their involvement in programs that provide less structured and more relaxed environments to cross racial /ethnic boundaries. As such, institutions may choose to direct their energies and resources to initiatives ... that offer opportunities for students to engage each other both formally and informally within the same space. (p. 36)

Intercultural student development is an important and worthy goal to help students develop their global-self. Successful intercultural communication has many barriers, including language capabilities, social standing, perceived ability, gender 
dynamics, racial differences, and cultural variations (Athanasios, 2005; Duţă, 2014). To develop the skills needed to acquire the mature attributes Perez et al. (2015) described as essential for improving understanding of cultural differences, and to overcome barriers to communication, it is important to have an intentional approach to program design and student learning. Understanding how students learn is the topic of the next section.

\section{Learning}

In the development of the global-self, the student learns something that changes how they interact with others; the change may be how a student views another culture, how they understand racism, or how they understand the difference between religion and spirituality, but the change only develops in the student because they learn something. This section provides a brief tour of experiential learning theories, relates them to Killick's (2015) suggestion that students need to interact with others to develop the global-self through intentionally designed delivery mechanisms, and reviews the research regarding how these interactions can be beneficial when they occur in the at-home environment.

\section{Experiential Learning}

Experiential learning in higher education often involves applying knowledge gained in a classroom setting to a workplace setting. New learning results from the application of the classroom setting in a different environment (Garraway \& Volbrecht, 2011). The transfer of knowledge from the educational setting to the workplace is not as simple; rather, it requires using different cognitive and social cues. For example, transferring the learning about soccer derbies from the classroom to the field involves both knowing the history involved with the clubs while also identifying the emotional 
elements to rivalries. In his study of bicycle racing officials, Grover (2014) found that referees identified three components of their experiential learning. In his study, entrylevel officials reported that they learned best from on-the-job training, working with others to improve their practice, and reflecting on their experience. that involved a shared experience with others and reflection as a learning device.

Experiential learning can be helpful in developing intercultural learning. In Allport's (1954) contact theory, key conditions for successful intercultural meetings (i.e., meetings which might reduce prejudice across the participants) included "“equal status contact between majority and minority groups,' 'the pursuit of common goals,' 'reaching below the surface,' 'contact that leads people to do things together,' and activity which is condoned by 'authority"' (Allport, 1954). This cross-group contact is frequently perceived as a remedy to cultural constructs of racism, prejudice, and discrimination (Ladda, 2014). Cross-group contact can be as simple as discussions on sociocultural issues which "have been linked to democratic outcomes such as a predisposition to making social change and changing attitudes about lifestyles and cultures" (C.W. Johnson, 2014, p. 151).

While cross-group interactions between international and at-home students might appear simple to facilitate, Ward (2001) reminded us that

research has shown that the presence of international students, even in large numbers, is insufficient in itself to promote intercultural interactions, to develop intercultural friendships and to result in international understanding. Rather, situations must be structured to foster these processes. (Key Findings, Strategies, para. 1)

Rogers noted "the importance of [the] inter-subjective encounter as a/the site for developing our understanding of the world and of ourselves within it. It is in such 
encounters that the participants ... found much of their own significant learning" (as cited in Killick, 2013, p. 723). The inter-subjective encounter, or encounters with coconstructed views of others (Killick, 2012), is an example of how experiential learning can help develop the global-self.

Our interactions with material and people are essential to learning, but without reflection, the learning is limited (Dewey, 1933). Reflection requires "assessing the grounds [justification] of one's beliefs" (Dewey, 1933, p. 9) and is beneficial for international students (Hellsten \& Prescott, 2004; C. W. Johnson, 2014) and at-home students (Chang, 2006). Reflecting on learning has been identified in multiple studies as an important part of shifting students' and staff members' consciousness to their development of global-self (Cho \& Yu, 2014; Deardorff, 2006; Hellsten \& Prescott, 2004; C. W. Johnson, 2014; Marginson, 2014; Mezirow, 1990).

Experiential education's value is demonstrated by Vygotsky's (1978) concept of the zone of proximal development. Vygotsky's zone of proximal development is the zone between what a student already knows and what the student could learn if helped by somebody who has additional knowledge. According to Vygotsky, learning can happen better in groups because implicit in groups is interaction and reflection between people of different zones. Vygotsky's concept suggested that students would become more interculturally competent if presented opportunities to learn from others with different cultural backgrounds.

Learning from others is essential to scaffolding theory, an application of the zone of proximal development. Scaffolding is when another person actively helps somebody move out of their current learning zone into their next zone by intentionally designing 
learning that bridges the gap from old knowledge to new knowledge (Austin, Orcutt, Rosso, \& Darling-Hammond, 2001). Applying this learning theory to the development of the global-self, Killick (2015) pointed out that while scaffolding can help a student develop intercultural competence, if the person supporting the learning brings the same sociocultural lens as the learner, then the learning would not be as effective. Learning, therefore, needs to be accompanied by multiple voices and perceptions when developing the global-self.

In short, "the most fundamental aspect of a successful inter-group contact situation is that it must involve people acting — doing something together, not just being co-present” (Killick, 2015, p. 63). Killick’s (2015) plea for interaction was essentially a plea for experiential learning, but reflection (Dewey, 1933) and support from others (Vygotsky, 1978) are also important to deepen the impact of experiential learning.

\section{Constructivist Learning}

Constructing our own knowledge is the core concept of constructivist learning and is an important part of the experiential learning process. Constructivist learning theory provides a contrast to realism's concept that there is one truth, which for much of education's recent history was the guiding principle driving the way teachers taught and students learned. According to realism, there is one set of truths, and the learner's role is to take a journey that leads them to a predestined end. In comparison, constructivism suggests that knowledge is contextual and relies on the experience and the interpretation of the learner. As such, constructivism modifies the way teaching is delivered and how programs are designed. Applefield, Huber, and Moallem (2000) suggested that "modern constructivist thought provides the theoretical basis for cooperative learning, project or 
problem based learning and other discovery oriented instructional approaches, all of which appeal to the powerful social nature of learning" (p. 39). Hein (1991), in a lecture on constructivism and its relationship to learning in museums, provided eight principles of the constructivist learning process:

1. Learning is an active process.

2. People learn to learn as they learn.

3. The crucial action of constructing meaning is mental.

4. Learning involves language.

5. Learning is a social activity.

6. Learning is contextual.

7. One needs knowledge to learn.

8. It takes time to learn.

Each of these learning principles applies to the way curriculum or programs are developed. For instance,

in constructivist and student-centered learning perspective, it is in group work that we might establish the most solid communities of practice and stimulate the coconstruction of knowledge as more-knowing peers scaffold others across their [zone of proximal development].” (Killick, 2015, p. 161)

The development of internal knowledge is not easy to observe or measure. But, as students play sports with each other and form communities, there is the opportunity to build knowledge — in this case, on their global-self — if they are primed for learning. The use of prompts, the diversity of players, and the opportunity for reflection all contribute to students creating their own interpretation of events and moving forward toward their sense of self-in-the-world.

One final way that students co-construct knowledge is through teamwork. Salas, Sims, and Burke (2005) identified a set of "Big Five" attributes necessary for successful teamwork: (a) team leadership, (b) mutal performance monitoring, (c) backup behavior, (d) adaptability, and (e) team orientation. Successful teamwork is an example of 
cooperative learning, which is an important part of constuctivist teaching. Teamwork is different than team identification, or how an individual develops a sense of "oneness" with an organized team (Gundlach, Zivnuska, \& Stoner, 2006). Successful teamwork requires a shift from individualism toward collectivism and an understanding that one is part of a shared goal. Shifting toward collectivism can help students construct their understanding of an experience and help them define its impact on their learning.

\section{Learning at Home}

While many students develop "a higher level of open-mindedness" (Twombly et al., 2012, p. 73) through travel abroad experiences, many students do not have the luxury to study abroad due to high cost, minority group marginalization, or fear of unfamiliar places (Soria \& Troisi, 2013). Thus, it is particularly important that environments are created for students to engage in activities on campus that develop similar internal changes as they would get traveling abroad. Building on the idea that "commonalities between domestic issues of multicultural education and global issues of internationalization are greater than their differences in today's world" (Chickering \& Braskamp, 2009, p. 30), Soria and Troisi's (2013) research argued that “internationalization at home activities can also promote students' development of global, international, and intercultural competencies just as effectively as - if not more effectively than-formal study abroad" (p. 273).

Soria and Troisi (2013) provided examples of at-home activity that included: (a) attending global or international focused lectures, workshops, or performances; (b) enrolling in courses with an international theme; or (c) residing in living-learning environments. These types of activities support the notion that colleges and universities 
should promote the social engagement between international and domestic students in and outside of classrooms (Hser, 2005).

One of the tools universities have used to increase at-home learning is through international students themselves. Urban and Palmer's (2014) quantitative study investigated how international students perceived they were being used as cultural resources at a large research institution in the Midwest. On every variable, international students wanted to be more helpful then they were being asked to be, and the "data indicate[d] that the international students in the study [were] not being actively engaged as cultural resources, and they [wanted] to be engaged to a much greater extent" (Urban \& Palmer, 2014, p. 319). Urban and Palmer (2014) concluded that "because cultural learning happens mostly through interpersonal relationships ... higher education institutions should seek multiple ways of facilitating meaningful interactions among domestic and international students" (p. 321), thus tapping into the international students' desire to help intercultural development and creating the environment for internationalization at home.

Learning is key to students developing the attributes of a global-self. Experiential learning often requires that the learner be in some form of relationship so that the learner has somebody with whom to create knowledge. While there are benefits gained from learning about internationalization and globalization through actual experience in another country, the research indicated that students can have similar learning outcomes at-home when environments are set up to facilitate interactions that require people to work together and use each other's knowledge. 


\section{Sport and Recreation for Social Change}

Collegiate recreation is one platform for increasing interactions to help students develop a global-self. Over $75 \%$ of students participate in collegiate recreation nationally; within that group, 34\% reported engaging in playing intramural sports (Forrester, 2014). Sports help mitigate conflict resulting from differing identities (Weiss, 2001). In healthy competition, people can become unified in playing and in exploring what it means to work with others toward a common goal. In unhealthy competition, sport is divisive, furthering a sense of "us" and "them" (Wann \& Grieve, 2005). Sport, as a concept and a practice, influences social change, including impacting how people feel about, and understand, their relationship to people different from them. International students' experience in sports in collegiate recreation impacts how students engage with the university. This study is interested in the intersection between those two areas.

\section{Sport and Social Change}

Sport is a major part of the cultural fabric of the world. An entire historical review of the role that sport plays in cultures around the world is beyond the scope of this research, but this section's review of its role in recent history helps place sports in the context of development of the global-self. In addition, a recently developed conceptsport for development and peace (SPD) — is introduced to explain sports' potential for inducing social change.

Four eras have defined the recent history of sports' impact on social change: (a) the "rational recreation" interventions of the middle and working class families in the late 19th century, (b) the "playground movement" of the early 20th century, (c) the worker's sports movement of the interwar period, and (d) the newest era of "midnight basketball" 
interventions supporting inner city youth of the last 15 years (Kidd, 2008). These

movements were part of general changes to social norms, which embraced-particularly in the United States-how sports could unify communities.

Multiple geo-political changes influenced how sports have been used for development and peace in "the end of the Cold War, the fall of apartheid in South Africa, the neo-liberal emphasis upon entrepreneurship and the mass mobilizations to 'Make Poverty History'" (Kidd, 2008, p. 370). Each of these events galvanized athletic activism in

the growing use of sport in the developed world to address "social problems," the increasing inclusion in human rights campaigns of the rights to sport, physical education and play ... the internationalization of feminist efforts to realize full opportunities for girls and women ... [and] the reassertion of the benefits of sport and physical activities through the world Health Organization, UNESCO, and other international bodies. (Kidd, 2008, p. 374)

Two distinct areas of sport for social change have emerged in practice: sport for development and sport for peace (Kidd, 2008). Sport for development focuses on how sport helps solve problems like AIDS education, landmine avoidance, or reproductive rights. In contrast, sport for peace is focused on conflict resolution, particularly ethnoreligious and ethno-nationalistic conflicts (Giulianotti, 2011); recent examples range from FIFA's No to Racism campaign to NFL and NBA players embracing the "Hands Up, Don't Shoot" movement.

In their examination of reconciliation through sports in South Africa, Höglund and Sundberg (2008) highlighted four different channels through which sport promotes reconciliation at political, racial, ethnic, and socioeconomic levels. First, they discussed the utilization of symbols and symbolic acts of reconciliation in South Africa's national 
reconciliation after apartheid, pointing to a number of different events, including a symbolic handshake between Nelson Mandela and the captain of the national rugby team, the flying of the Olympic team in a plane painted in the design of the national flag, or the hosting of the African Nations Cup of Soccer that helped promote the idea that "black athletes can succeed just as well as white ones" (Höglund \& Sundberg, 2008, p. 808). Second, Höglund and Sundberg (2008) highlighted the application of sport policies to create fair representation in South African sports team. South African governing bodies adopted a policy of "sport unity" that was "aimed to create a completely new system [of team selections] based on unity, equality, empowerment and representation" (Höglund \& Sundberg, 2008, p. 809). These policies, while not quotas, enforced the expectation that teams would be more representative of the population of South Africa. While in the case of South Africa there remain significant challenges, the system of sport unity did change behavior and spark dialogue through sport.

Höglund and Sundberg (2008) also argued that sports could help break down stereotypes and negative attitudes through inter-communal sport initiatives, noting that "through tournaments and leagues people have a chance to interact with members of other communities" (p. 811). Keim (2003) confirmed that these interactions contributed to increased acceptance of the other, while noting that having trained instructors helped these interactions to be more effective. It cannot be ignored, however, that sport can work in opposition to the goals of unification by creating tribalism, lowering trust, and increasing conflict. The very nature of sport, teams, and winning can create tension if not appropriately channeled. 
Höglund and Sundberg's (2008) final channel for reconciliation through sport was individual development. In their conclusion, they argued that this development "rests mainly on the creation of opportunities for those at risk of marginalization or of a life in conflict with the rest of society" (Höglund \& Sundberg, 2008, p. 813) and pointed to successful soccer and basketball programs that have contributed to reduced crime, increased understanding of health issues, or even helping kids overcome trauma. These four channels have parallels to students developing their global-self through acting-inthe-world by participating in events that are meaningful to them or by having practices that create more equitable conditions. These four methods of reconciliation contribute to students knowing their self-in-the-world through participating in sport activities with others or helping students heal themselves. These methods influenced the design of this study.

\section{Sport and the Other}

Creating winners and losers, an inherent outcome of sport, undoubtedly creates a sense of the "other." Turned negatively, losing can create feelings of anger or frustration and threats to identity (Wann \& Grieve, 2005). Depending on the results of a competition, participation in sports can create a perception that one team, or even one person, is better than the other (Wann \& Grieve, 2005). These perceptions can be true for both spectators (Kerr, Wilson, Nakamura, \& Sudo, 2005; Wann \& Grieve, 2005) and for participants (Kerr \& Males, 2010). There is a gap in the literature about how interactions between teams and individuals occur between international and domestic students in a collegiate recreation context. To address the gap, it is helpful to review the literature on the experience of immigrants, as they often have similar attributes to international 
students and there has been more research done on their relationship to sport. Immigrants and international students face similar challenges in their new countries, including finances, language barriers, social isolation, and cultural dissonance (Hazen \& Alberts, 2006; Kim, 2012). For immigrants, participation in sports has reduced the perception of us-versus-them (Allen et al., 2010; Coryell et al., 2012; Kim, 2012; Stodolska \& Alexandris, 2004). Knowing that the experiences between immigrants and international students are similar allows us to apply the research to the discussion about how to minimize the sense of the other.

Addressing the perception of the other is core to social identity theory (Tajfel, 1982; Tajfel, Brown, \& Turner, 1979). In social identity theory, people often behave in defense of their constructed identities - the identities that they have chosen to take onby adopting strategies that maintain comparisons favoring the in-group and the self (Hogg, Terry, \& White, 1995). Group identification ranges from the political party with which somebody identifies to the gender identity one has or what sports team one cherishes. By deconstructing these identities, a person can move beyond feeling threatened when they are in the minority and move toward an understanding of commonalities. Universities working toward internationalization are often trying to address outcomes that reduce the prevalence of the group identity (Killick, 2015).

Because one of the cores to Killick's (2015) global-self is understanding your own self-in-the-world, knowledge about one's racial or ethnic identity is an important aspect for students to examine in their development. While stereotyping of varsity athletes often carries negative connotations over to off-the-field activities (Beamon, 2014; Feltz, Schneider, Hwang, \& Skogsberg, 2013; Simons, Bosworth, Fujita, \& Jensen, 
2007), participation in student affairs activities like recreational sports in higher education can help at-home students understand their racial and ethnic identity (Karkouti, 2015; Pieterse et al., 2010).

While the experience of immigrants and international students is similar, and the development of racial and ethnic identity in higher education is important, the research on the nexus of international students, sport, and the development of racial and ethnic identity is limited. The most relevant research is the Allen et al. (2010) study of sport and its role in socialization and maintenance of cultural identity for international students, which found that "International students participating in [recreational sports] emphasized adaptation to a multicultural environment through sport over cultural maintenance through sport” (p. 431). However, the study did not address students' perception of other's, or their own, global-self— two aspects this current study investigated.

One final concept in sport that helps students learn about their relationship with the other is teamwork. Salas et al. (2005) identified a set of Big Five attributes necessary for successful teamwork: (a) team leadership, (b) mutal performance monitoring, (c) backup behavior, (d) adaptability, and (e) team orientation. These five attributes rely on the "development of shared mental models, achievement of mutual trust, and engagement in closed-loop communication" (Salas et al., p. 559) to be effectively implemented. Teamwork is different than team identification, which is how an individual develops a sense of oneness with a particular organized team (Gundlach et al., 2006). Successful teamwork, on the other hand, requires a shift from individualism toward collectivism and an understanding that one is part of a shared goal. 


\section{Collegiate Recreation and International Students}

There is a lack of research on international student development in collegiate recreation and in addressing how recreation can help students develop their global-self. While international students frequently participate in collegiate recreation, firm participation numbers are hard to find and there has been limited research done specifically in the collegiate recreation context about the engagement of international students. A review of the most cited studies sheds light on some of what is known so far, including perceived barriers, cultural differences, and rates of engagement.

In their semi-structured interviews, Li and Stodolksa (2006) found there were several barriers to Chinese international students' participation in leisure recreation as an activity when studying in the United States. For these Chinese students, their temporary status of residence and their maximization of academic time impeded their ability to interact with and benefit from participation in recreational activities. Additionally, Chinese students' sense of transnationalism, or their "maintain[ing] social, political, and cultural linkages to their countries of origin and to other members of the emigrant community" (Li \& Stodolksa, 2006, p. 40), contributed to their inability to participate in recreation.

Guo and Ross (2014) studied Asian international students participation specifically in a campus recreation setting using an online survey to ascertain answers to four questions: (a) the sports participation status of Asian international students, (b) barriers, (c) benefits obtained from participation in campus recreational sports, and (d) demographics (p. 58). Guo and Ross found that Asian students who did engage in recreational sports usually did so more for the physical benefits than the social benefits. 
Asian students were more likely to participate in informal sports individually or with their friends of the same culture rather than in structured sports programs. Intramural sports were the least popular sports programs for Asian international students. Intramural sports provide opportunity for interactions across demographic sectors, thus this is a missed opportunity for Asian international students in the development of the global-self.

Heo and Lee (2007) studied Korean college students playing recreational basketball at an American university. Using a purposive sampling strategy, seven indepth, semi-structured interviews were conducted to understand how those from a collectivist culture (Korea) perceived their participation in "serious leisure" (Heo \& Lee, 2007). While not international students, the lens the authors used in studying the cultural impacts helps to understand how sports impact different cultures. The results of the study demonstrated the complexity of linking participation in serious leisure and the development of the global-self. On one hand, participants became "involved with basketball because it enhanced their sense of self" (Heo \& Lee, 2007, p. 148), one of Killick's (2015) main attributes of global-self. On the other hand, the participants reported they "were closely bonded with other Korean students through basketball, but became isolated from the host culture" (Heo \& Lee, 2007, p. 148), an attribute that detracts from the development of the global-self. Despite these contradictions, these students still demonstrated attributes of improving their self-in-the-world through basketball's positive impact on their "self-enrichment, social belonging, and enhancement of self-image" (Heo \& Lee, 2007, p. 147).

Shifman, Moss, Andrade, Eichel, and Forrester (2011) used quantitative methods to investigate the barriers for students participating in intramural sports in a Canadian 
university. International students were a specific subset of their study. Many international students reported intrapersonal constraints to participating that were centered on a lack of skills or a lack of personal confidence. The study also found many structural barriers to participation, which most frequently included a lack of time due to "work, school, or family" (Shifman et al., 2011, p. 10). Other studies have found that the focus on work and school cuts into time spent on sport or recreation activities (Guo \& Ross, 2014; Henderson, 2014; Heo \& Lee, 2007; Li \& Stodolska, 2006).

Allen et al. (2010) were interested in examining how international students at American universities could use sports as a "vehicle for socialization and maintenance of cultural identity" (p. 421). Using a scale developed to measure "socialization, cultural maintenance, and adaptation to multicultural environments through sport" (Allen et al., 2010, p. 427), the authors analyzed 240 online surveys received from international students at four American universities. Their findings concluded that for international students who participated in sport, adaptation to a multicultural environment was more important than cultural maintenance, and that men were much more interested in cultural maintenance than women. Cultural maintenance, while supporting international student success, could be a barrier to the development of the global-self if it inhibits the adaptation or engagement with a broader multicultural environment. In fact, Glass, Gómez, and Urzua (2014) found a clear relationship between a student's region of origin and their ability to engage with an institution via participation in a recreation program. Non-European students had more difficulty adapting and feeling connected to their college than their European counterparts. These students also tended to participate in 
activities with their own national peers; this was especially true for Eastern/Southeastern Asian students.

Allen et al. (2010) found another statistically relevant aspect to participation in sport — there was a sweet spot. Too few hours or too many hours participating resulted in less successful adaptation; the ideal number of hours to participate in sports is between four and eight times per week. Research has found that most international students fall into a lower category (Yoh, Gordon, \& Yang, 2008).

Shifman et al. (2011) studied international students and intramural sports and concluded that international students had more intrapersonal and interpersonal barriers limiting their participation than non-international students. There is little research that discussed how these barriers to participation might be linked to developing personal attributes such as those associated with the global-self. There is a gap in the literature about how sports in a collegiate recreation setting might influence students' development of a global perspective or a global-self, which this study addresses.

\section{Competencies}

Student affairs professions use competencies to assess and describe characteristics associated with successful professionals (American College Personnel Association [ACPA] \& National Association of Student Personnel Administrators [NASPA], 2015; National Association of Colleges and Employers [NACE], 2013; NIRSA, 2017). Competency attainment often measures the acquisition of beginner, intermediate, or advanced skill levels (Herdlein, Riefler, \& Mrowka, 2013). While using competencies to measure professional excellence is not universally accepted (Eaton, 2016), they remain a tool that the profession uses to set standards of performance and behavior. 
Skills perceived as important to student affairs professionals often depend on who you ask and the respondent's professional level (Burkard, Cole, Ott, \& Stoflt, 2005). Herdlein's (2004) survey of chief student affairs officers indicated that there was high satisfaction with the interpersonal development that new professionals demonstrated and overall satisfaction with intrapersonal development. Interpersonal skills were perceived as highly important for the new professional to have, but intrapersonal skills (those related to self-in-the-world) were not viewed as equally important. Almost a decade later, Herdlein et al.'s (2013) meta-analysis of student affairs competencies revealed that while knowledge and skills around multicultural and diversity issues remain highly important, there has been a shift toward focusing on administrative functions such as strategic planning or budgeting and away from competencies that involve human facilitation. This shift away from developing skills in working with fellow humans is problematic in a global world.

NIRSA, the professional association for collegiate recreation, has identified eight competency areas for collegiate recreation professionals that include both administrative and developmental areas: (a) Programming, (b) Philosophy and Theory, (c) Personal and Professional Qualities, (d) Legal Liabilities and Risk Management, (e) Human Resources Management, (f) Facility Management Planning and Design, (g) Business Management, and (h) Research and Evaluation. These competency areas form the backbone of a professional registry program that provides "a framework for lifelong competency based professional development and inspires purposeful acquisition of new skills" (NIRSA, 2017, p. 1). NIRSA commissioned a report using the Delphi method that validated the competencies (Haines \& Forman, 2009). 
Appendix A includes descriptions of each of the NIRSA core competencies, providing specific details of the competency areas that support the importance of development of the attributes of the global-self. Three of the eight NIRSA competencies address professional standards related to the development of the global-self: (a) Programming, (b) Philosophy and Theory, and (c) Personal and Professional Qualities. A brief description of each of those competencies helps provide understanding of their relevance to this study.

\section{Programming}

The delivery of programs is a "core purpose for collegiate recreation professionals," and professionals "need to be able to develop and administer high quality programs and to appropriately apply associated resources” (NIRSA, 2017, p. 2).

Programs vary from school to school but often include activities developed in areas such as intramural sports, group exercise classes, student organizations, or outdoor adventures. Successful programming is integral to student engagement and the use of experiential education as a learning tool. This competency area includes 14 measurable components broken down into basic, intermediate, and advanced levels of skill acquisition. Six of these component areas provide a rationale for professionals in collegiate recreation to promote and understand the development of the global-self: (a) student learning and development; (b) teaching and training; (c) program development; (d) leadership; (e) equity, diversity, and inclusion; and (f) global perspective.

\section{Philosophy and Theory}

The philosophy and theory competency "involves knowledge and skills that connect the philosophy and theory to professional practice ... through the use of existing 
frameworks and the development of new frameworks" (NIRSA, 2017, p. 2).

Understanding intercultural development and the role it can play in social change through sport is an important part of the philosophy and theory competency area. The competency descriptions illuminate the philosophies and theories in which collegiate recreation professionals can learn skills specifically related to recreation in a higher education setting. The philosophy and theory competency area has a total of nine measurable components broken down into basic, intermediate, and advanced levels. The four most relevant components that provide rationale for professionals to engage in the development of the global-self are: (a) student affairs issues and theories, (b) student development theory, (c) trends in recreational sports, and (d) equity and diversity. Understanding the philosophy and theories in higher education equips professionals to move forward in promoting the concept of the global-self.

\section{Personal and Professional Qualities}

NIRSA (2017) has identified that the personal and professional qualities

competency area

involves the knowledge, skills, and attitudes to inform individual conduct and apply these tools toward productive work and interaction; awareness of one's own areas of strengths and areas for development; be committed to lifelong learning; to assimilate integrity and ethics into all aspects of personal and professional self. (p. 2)

Individual awareness of one's own need for development is key for movement toward the global-self. This competency has nine measurable categories broken down into basic, intermediate, and advanced levels. Herdlein et al.'s (2013) observations noting the shift away from human facilitation as an emphasis in new student affairs professionals' preparation suggest a need for recalibrating this skill based on the 
changing demographics of higher education and movement toward a globalized world.

The personal and professional qualities competency area has three categories where there are implications for the development of the global-self: (a) adaptability; (b) equity, diversity, and inclusion; and (c) global perspective.

\section{Conclusion of the Literature}

Starting with a review of Killick's (2015) global-self theory, the literature review included sections relevant to the proposed case study. How institutions shape their environment and shape their curriculum impact how students interact with the university. While the formal curriculum sets the groundwork for learning, the literature suggests that the informal and hidden curricula powerfully influence how students perceive their engagement. The examination of student development theories help explain how student development theory connects to intercultural competence and how the intersection of student development and intercultural competence is an important component of the global-self.

Student development and intercultural competence may increase when students participate in experiential learning combined with reflection and support from peers. The literature on the role of sport and recreation for social change reviewed how experiential learning benefits from intentionally designed interactions. The current literature on collegiate recreation and its impact on students' global-self supports the need for further work on linking recreation, sport, and social change through intercultural competence development. The literature on the power of sports in creating social change supports the case that the development of the global-self is possible when the right conditions are 
present. This chapter concluded with a review of the NIRSA professional competencies, a framework for assessing the quality of professional development in collegiate recreation. 


\section{CHAPTER 3: METHODS}

This methods chapter provides an overview of the research approach and design of the completed study. The first section of this chapter provides an overview of the purpose of the study followed by a description of the research perspective, including the conceptual framework and study type. Details on the procedures used in the study follow, including the target population and selection, research organization, data collection and analysis techniques, and care for participants and data. Finally, the chapter concludes with an examination of the role of the researcher, the proposed transferability of the study, and a summary.

\section{Purpose of the Study}

Maxwell (2013) reminded us that the "function of [the] research question is to explain specifically what your study is intended to learn or understand" (p. 75). He clarified that the learning is both intellectual (i.e., what we hope to understand by doing the study) and practical (i.e., what we want to accomplish); both are important tasks to accomplish in the formulation of the research question. For this study, I was interested in the development of the global-self through participation in sports. The study had two goals: the first goal was to understand how students perceived the development of their global-self through participation in an intramural sport; the second goal was to understand how students experienced the intentionally designed intramural program so that the interventions could be replicated.

In developing the research question, Maxwell (2013) cautioned the qualitative researcher to intentionally choose the type of question used. The research question, because it focused on the perceptions of the participants, was constructivist in nature. 
Using a constructivist lens allowed for the observation of phenomena and the data collected about the phenomena to be "used crucially to develop and test ideas about the existence and nature of the phenomena" (Maxwell, 2013, p. 80). Practically, the research questions for this study allowed for the participants in the study to speak about how they experienced their interaction with others in intramural sports. The research questions for the study were:

- How do students participating in a collegiate intramural soccer program perceive the development of their global-self after experiencing interventions intentionally designed to enhance discussion of, and reflection on, attributes of the global-self?

- How do students experience an intramural soccer program designed to encourage the development of global-self characteristics?

\section{Research Perspective}

In this section, I review the research framework, connecting Killick's (2015) global-self and Allport's (1954) experiential learning theories to highlight the intersection of these two theories of learning as they form the core of the implemented intervention. I then discuss the type of study that I conducted and discuss how community action research influenced the study. This section ends with a description of transferability.

\section{Framework}

The conceptual framework for this study was grounded in Killick's (2012, 2013, 2015) concept of global-self. Killick's model suggests that interaction between international and domestic students is critical for the development of commonalities. The global-self model relies on students recognizing that the other is, in fact, equally human. 
This recognition requires that students understand their self-in-the-world and develop their ability to act-in-the-world. Killick (2015) was very clear that for the "global student, this means experiencing university life and learning in inclusive and equitable spaces which allow for and enable interactions across cultural borders and beyond narrow conceptualizations of us and them" (p. 33). To activate this type of environment, this study relied on experiential learning that had students engaged in "active, hands-on learning and reflection on their experiences, [whereby] students [were] able to bring theory and classroom knowledge together with practice" (Colby \& Sullivan, 2009, p. 29). I theorized that with the appropriate prompts, students would have experiences that created in them a new sense of who they are and a new awareness of the others with whom, and against whom, they were playing.

Killick (2015) offered two important perspectives that informed the methodology of this study. First, he proposed that developing our practice and associated learning environments to enable our students to get used to each other and establishing a focus on community building are ... fundamental to reducing identity threat and building the kind of personal security amongst alterity from within which more transformative learning might take place. (p. 130)

Second, Killick reminded us that one of Allport's (1954) experiential learning tenants was that to reduce prejudice through intercultural encounters, participants in learning must have equal status. This equal status is developed when there is "an unambiguous message of equality to students, a message that promotes an environment which embraces cultural diversity and within which all students feel they have something significant to contribute" (De Vita, 2000, p. 175). These two perspectives work with each 
other in an iterative cycle; building communities based on a sense of equality helps students to get to know each other, which results in better communities.

\section{Case Study and Community Action Research}

The best assessment measures of intercultural competencies as an outcome of internationalization are recommended by Deardorff (2006) to be "primarily qualitative in nature, including the use of interviews, observation, and case studies, as well as the possible use of standardized competency instruments" (p. 258). Deardorff stressed that the assessment of intercultural competence, like the assessment of the global-self, is difficult due to the multiple variables that impact students' self-identity. Deardorff's research noted that while mixed methods approaches can be helpful in assessing intercultural competence, case studies and interviews are most often viewed as the tools of choice in assessing intercultural competence. The case study, a form of qualitative research, was my method of study.

Case studies can be differentiated into three forms (Creswell, Hanson, Plano Clark, \& Morales, 2007): intrinsic, instrumental, and collective. Intrinsic case studies typically focus on a particular case; for instance, how does one student perceive they were treated in an intramural league, since that particular case "presents an unusual or unique situation"? (Creswell et al., 2007, p. 247). An instrumental case study “focuses on an issue or concern and then selects one bounded case to illustrate [the] issue" (p. 246) while a collective (or multiple-case) case study "selects one issue or concern but also selects multiple case studies to illustrate the issue" (p. 246). An instrumental case study was used in this study because of the interest in a particular issue in a particular setting: 
how intramural sport programs can be designed to support participants learning about their global-self at an urban university.

While the overarching study approach was a case study, the procedures for the study also pulled from community action research. Community action research is often used in the context of solving "difficult community problems" (Ozanne \& Anderson, 2010 , p. 123). The development of the global-self and all it requires is a community problem. This case study relied on three of the core components of community action research. Each component improved the methodology used to understand the problem-inpractice.

First, Ozanne and Anderson's (2010) study included "multiple partners from the community in the research process" (p. 124). During the study, I worked with the student staff during their training to teach them about the global-self. This training had two distinct components: one for student leaders and one for regular student staff including referees and site supervisors. In the summer prior to the intramural league, I worked closely with the two student coordinators to inform them about the proposed intervention and then to collectively identify strategies, dates, and times for the training of the rest of the student staff and players. Together, we developed the training for the intramural staff and the team captains. We then worked together to create pre-game scripts and methods for the referees to use when they were doing the pre-game interventions on the soccer field. Second, the study tried to "solve practical problems and improve the well-being of people, particularly those who suffer from enduring social inequities" (Ozanne \& Anderson, 2010, p. 124). The study design helped solve a practical problem in collegiate recreation by improving the overall well-being of students through the development of 
their intercultural competence. Third, the study approach relied "on community education and empowerment and thus encourage[d] people to learn new skills, reflect on their social and economic conditions, and act in their own self-interest" (Ozanne \& Anderson, 2010, p. 124). The use of community action research techniques provided a unique perspective to the research problem, supported Killick's (2015) development of the global-self and Allport's (1954) experiential learning, and addressed some of the challenges of doing research at my own institution.

\section{Procedures}

This section describes the procedures, beginning with a description of the study context and the target population. The overall setting for the case study is described and the detailed interventions outlined. Following this, the timing and methods of the data collection, and the data analysis, are described. Finally, this section describes how I obtained informed consent, protected the rights and well-being of the participants, and maintained the data securely.

\section{Study Context and Target Population}

Soccer involves a broad range of cultures and languages and was a good sport to use in this study as "involving mixed teams help(s) to build inclusive social capital across the communities, by forging relationships founded upon informal, practical cooperation" (Pickering, 2006, p. 218). In case studies, all aspects of the case can have an impact on the study, and the researcher needs to understand the experiences of each of the participants. In this case, the study took place at a large, urban, research university on the West Coast. The intramural program averages 450 unique students participating annually. The target population for the study had two student categories: staff and players. The 
intramural staff numbered 13 students, including two student coordinators, three event supervisors, and nine referees; there were a combined 282 players who participated in the competitive or recreational intramural soccer leagues.

\section{Research Procedures}

Bounding this case study was the preparation for, and the implementation of, an 8-week intramural soccer league. Appendix B includes a detailed timeline of the research schedule. The study relied on four interventions that modified the practices of past intramural soccer seasons. While the collegiate recreation profession often highlights the role that intramurals play in improving sportsmanship or tolerance in players, most programs and research focus on creating opportunities for employee development rather than modifying participant behavior. The procedures used in the study attempted to address both participant and student staff development.

Based on Keim's (2003) finding that sport education in schools contributed positively to reducing intolerance when there were trained instructors and when language barriers were removed, the first intervention of the study included modifications to the intramural student staff training. The first modification to the student staff training was the implementation of a learning module to train student employees about intercultural competence and the global-self. In developing the learning module, I examined both the demographics of the prior year participants and data available from new international student enrollment to inform the focus of the training. The student coordinators and researcher co-led the training in the intramurals office located in the Student Recreation Center. There were eight student employees present. 
The student staff training was organized in two parts: a 45-minute classroom session and a 45-minute informational session with two international students. The first portion of the classroom session was a simulation of the pre-game questions. Each staff person introduced themselves, said where they were from, described their role on the staff, spoke about their familiarity with soccer, and answered the question, "What is your favorite Olympic sport?" After students completed their introductions, I introduced the concept of the global-self via a PowerPoint presentation. The presentation concluded with staff, in small-groups, actively reflecting on one statement and one question. First, the staff discussed the meaning of the statement, "Global students and global selves need an equally skeptical stance toward their own way of being as they have toward those of others," and then the staff answered the question, "What are the tools you identify as needing to be successful?" A short discussion about their answers followed.

The next step in the classroom session was watching three video clips of soccer "derbies." Soccer derbies are rivalry games usually between two teams from the same city. Each team in a derby often has an identity that followers embrace. These identities range wildly, but often include opposing personal attributes such as: religious beliefs (Celtic vs. Rangers), socioeconomic status (Boca Juniors vs. River Plate), or political beliefs (Real Madrid vs. Barcelona). After the staff watched the videos, they were required to reflect on what they watched by discussing the various derbies and how they related to their understanding of international students. Finally, two international students gave a 45-minute session about soccer and their country (see Appendix C), which was followed by a question and answer session between the international students and the staff. 
The second intervention of the study was a modification of the team captains' meeting. The first step in this modification entailed co-creating with the student staff a brief learning module about intercultural competence and the global-self to present to the captains of the teams. This learning module was used at the mandatory captain's meeting where the captains learn the rules of the league and the expectations of the program. The learning module introduced the concept of the global-self to the captains and informed them of the new pre-game practices.

The third intervention was the implementation of the referee script and pre-game questions in actual game situations. The script and pre-game questions were created with the two student coordinators to encourage humanization, build community, and spark reflection (see Appendix D). The specific changes from previous intramural seasons included

- addressing the entire set of players, rather than just the captains, at a mid-field pre-game meeting;

- using a pre-written script that introduced the concept of fair play and attributes of the global-self instead of simply reviewing the rules of the game; and

- a round-robin introduction of all players and staff where they introduced themselves by name and country of origin, and then answered a question intended to create self-reflection and connection between players.

The use of the pre-game script and the questions started during the first series of official games after the first week of practice games. I accompanied the referees during the first games to observe player and student staff reactions. 
The fourth intervention of the study included two social events created to “promote ... informal interactional diversity based on common identities and aspirations" (Caruana \& Ploner, 2010, p. 100). Baxter Magolda (2003) noted that "when dialogues can be ongoing mutual exchanges within already existing student groups they have the power to increase understanding of other cultures and increase students' confidence in inter-cultural dialogues" (p. 238). Leask (2009) noted that this required "purposeful planning." The intramural staff and researcher co-created the outline of the social event. This served two purposes: (a) the student staff helped identify the best time and place to do the intervention; and (b) they experienced how to design questions to inspire inclusion, fair play, and global perspective.

The post-game social event occurred on two different nights. During the postgame intervention, students demonstrated both great interest and great indifference toward the activity. Students who chose to participate were provided with one of seven questions to answer with a player from another team in order to get a piece of pizza. The questions were designed to be simple while encouraging a broader global perspective. On the first night, the weather was cold and, because the field did not allow food to be eaten on it, players had to walk 35 yards to receive their pizza. This intervention did not produce the hoped-for number of interactions. Learning from that experience, the second post-game intervention had a much better result. In the second post-game intervention, students received information when they checked in for their game and thus had more time to discuss the content of the questions. Because their reward for participating (candy) was right in front of them, more students interacted with somebody from another 
team than when the reward was 35 yards away. The questions were modified and related to the cultural history of Halloween or similar holidays from countries around the world. See Appendix E for the list of questions for both nights.

\section{Data Collection}

To answer the research questions through an instrumental case study, I collected five types of data: observations, field notes, surveys, semi-structured interviews, and sportsmanship scores. Data collection started almost immediately with observations and field notes of early interactions with the intramural student staff. Observations and field notes of all activities supplemented the online survey and the semi-structured interviews completed at the end of the study. Sportsmanship ratings for each team were collected each week of the intramural season and compiled into season averages at the end of the season.

Glesne (2016) identified numerous opportunities and strategies for observation, including the study of the setting, the noting of events details, and participant interactions and gestures. Observations were completed during the student staff training, the captains' meeting, pre-game introductions, and the games. These observations provided insight into how students experienced the interventions. By observing reactions, listening to conversations, and watching the interactions between students, I formulated opinions of participant satisfaction and levels of engagement, which contributed to my assessment of the participant experience. I took descriptive field notes on seven nights and observed over 20 games to aid in the neutrality of my recording of the case study details. I reviewed videos of the pre-game meetings to analyze the reactions and responses 
individuals had to the pre-game scripts and questions. Analytic notes, written at multiple points in the process, helped me to better understand what I observed and to sort through my initial findings and ideas.

At the completion of the fifth week of the season, all 282 players and student staff were sent an email that described the study (see Appendix F) and included the informed consent form (see Appendix G) and a link to an online Qualtrics survey. During the sixth week of the season, a small poster was attached to the check-in table at the intramural site reminding students that the survey was available for them to take. Students received a quarter sheet handout after their game that provided a web address to the survey, a reminder of the survey purpose, and information about the thank you card. A follow-up email was sent to all players and student staff two weeks after the initial email. A final email reminder was sent on the closing date of the survey. Student employee participation was voluntary, but they could complete the survey during work. The first 30 survey respondents received a $\$ 10$ gift card to thank them for their participation.

The survey drew inspiration from the Global Perspective Inventory (Braskamp, Braskamp, Merrill, \& Engberg, 2008) and included demographic questions and openended questions (see Appendix H). The survey encouraged students to reflect on their experience of the intramural season (Mezirow, 1990). Survey results assisted me in understanding students' perception of their global-self and their experience with the intramural season.

To identify study participants to interview, the final question in the survey asked participants to identify if they would participate in an in-depth interview. Once the survey closed, systematic random sampling was used to sort the data to identify students to 
interview. To clean the data, respondents were first sorted as either student staff or player. Players were further sorted into groups of recreation and competitive players. From each list of groups, every fourth person (starting with the fourth initial reply) was emailed to inquire about their interest in being interviewed and were given five days to confirm their interest. I did not initially receive enough replies to meet the goal of eight players and four student staff. Subsequently, I revisited the list and once again selected every fourth player (this time starting with the third reply). Through this process, I identified eight players to interview and I completed seven interviews. Two additional emails were sent to student staff to reach the goal of four student staff who were willing to be interviewed. A professional colleague, who works in Academic Affairs and who was a third-year doctoral student completed two interviews with student staff to assist in minimizing the effects of backyard research. Difficulties in scheduling resulted in the professional staff only completing two student staff interviews.

The semi-structured interviews consisted of a core of eight questions designed to improve the researcher's understanding of the participant's development of global-self and their experience of the intramural season (see Appendix I). Seven players and two student staff completed interviews. Table 2 shows the demographics of the interview participants.

All the interviews except one took place in-person. One player interview took place over Skype. Digital recordings of each audio interview were professionally transcribed. The interviews lasted between 24 and 40 minutes. Participants received a $\$ 20$ gift card to thank them for their in-depth interview. 
The intramural student staff assigned each soccer team weekly sportsmanship scores after they played. The intramural student staff also collected sportsmanship scores for the flag football and volleyball leagues occurring during the study window. The aggregated end-of-season sportsmanship scores were averaged for each league. Data for the prior year's soccer, flag football, and volleyball intramural leagues were compared with the study year. Table 2

In-Depth Interview Participant Demographics

\begin{tabular}{|c|c|c|c|c|c|}
\hline Name & $\begin{array}{l}\text { Country of } \\
\text { Origin }\end{array}$ & Race & $\begin{array}{l}\text { Year in } \\
\text { School }\end{array}$ & $\begin{array}{l}\text { Gender } \\
\text { Identity }\end{array}$ & Role \\
\hline Amine & Algeria & $\begin{array}{l}\text { North } \\
\text { African/White }\end{array}$ & $\begin{array}{l}\text { Grad } \\
\text { Student }\end{array}$ & Male & Player \\
\hline Harry & United States & White & $\begin{array}{l}\text { Grad } \\
\text { Student }\end{array}$ & Male & Player \\
\hline Helen & United States & White & Sophomore & Female & Player \\
\hline Sam & United States & Biracial & Senior & Male & Player \\
\hline Alexis & United States & Hispanic & $\begin{array}{l}\text { Grad } \\
\text { Student }\end{array}$ & Female & Player \\
\hline Quang & Vietnam & Asian & Senior & Male & Player \\
\hline Ned & China & Asian & Sophomore & Male & Player \\
\hline Edward & United States & White & Senior & Male & Staff/Player \\
\hline Nesto & USA/Spain & Hispanic/White & Senior & Male & Staff/Player \\
\hline
\end{tabular}

\section{Data Analysis}

Data analysis occurred during all phases of the case study, often resulting in an circular process where interventions were observed, modified, observed, solidified, and observed again. As a community action research project, collaboration with and observation of study participants allowed for constant data collection. Interactions with study participants encouraged reflection and modification to the study design resulting in 
improved interventions. Over the course of eight weeks, field notes and subsequent analytic notes documented observations and initiated thinking about what I was observing. Each data source built on the prior one to inform the direction of the analysis. Observations and field notes informed the initial review of the on-line survey which subsequently informed the in-depth interviews. The data was reviewed in a continuous, iterative method to arrive at the themes and conclusions presented.

The analysis of the online survey was critical to understanding the student experience of the intramural program and the students' perceived development of their global-self. The answers to the survey were qualitative, resulting in a body of text requiring interpretation. One method of interpreting text is the use of coding. Coding is a process used to analyze qualitative data that often results "in a word or short phrase that symbolically assigns a summative, salient, essence-capturing, and/or evocative attribute for a portion of language-based or visual data" (Saldaña, 2016, p. 4). Coding was essential to the analysis, and caution was taken to avoid placing inappropriate weight on individual words (St. Pierre \& Jackson, 2014). To initiate the coding, I used a Wordle. A Wordle is a computer-generated visual of the most frequently used words in a section of text and is a good supplemental tool to initiate coding (McNaught \& Lam, 2010). The resulting images provided a quick high-level overview of participant responses and placed frequently cited words together enabling initial identification of important words.

Creating Wordles that helped the analysis required multiple steps. While Wordles automatically remove commonly used words (e.g., the, and), the survey responses needed further cleaning to ensure spellings were correct and to align grammatical use. Initially, the Wordles had 150 words; because these Wordles had so much data, subsequent 
Wordles were reduced to 100 words and examined for any changes in words that stood out. In this process, axial coding helped to reorganize the data set by crossing synonyms out and removing redundant codes so all the best representative codes were left (Saldaña, 2016). After reviewing these results, forms of words emerged that needed examination of their context. For example, the word "play" occurred in "plays," "played," "playing," "player," and "players." A word count conducted for each question made it easy to identify the multiple forms of the use of a word. After reviewing the visual representation and the word count for each question, examination of each instance of a key word identified in the text ensured appropriate understanding of the context in which the word appeared (Ryan \& Bernard, 2003). If the use of the word had relevance to the research question, the larger text in which the word appeared was copied and categorized for further analysis. Each survey question was analyzed this way. After analyzing the responses to each question, I did a similar analysis of the totality of the responses to review the key words that emerged across all answers regardless of the prompt. These initial results resulted in several concept codes, suggesting "an idea rather than an object or observable behavior" (Saldaña, 2016, p. 119). Examples of these concept codes included ideas such as knowing, respect, and caring.

After completing the initial concept codes based on the survey data, I moved on to analyzing the transcribed in-depth interviews. These interviews provided more context than the survey responses and were initially analyzed using eclectic coding (Saldaña, 2016). Eclectic coding is an appropriate choice to use "for virtually all qualitative studies, but particularly for beginning qualitative researchers learning how to code data, and studies with a variety of data formats" (Saldaña, 2016, p. 213). Eclectic coding typically 
uses two or more first-cycle coding methods. The first-level coding of interview data used holistic coding to identify basic themes. Following holistic coding, provisional coding was used to look for specific self-in-the-world or act-in-the-world attributes such as humanizing or community that had been identified in the survey responses. Following these two coding methods, causation coding helped examine why people think they do or do not have a global perspective and identified some of the reasons participants perceived they had developed attributes of a global-self. Additional second-level coding was used to get results that were "reorganized, and reconfigured to eventually develop a smaller and more select list of broader categories, themes, and concepts, and/or assertions" (Saldaña, 2016, p. 234). To make sense of the results of the coding, codeweaving - the "actual integration of key code word and phrases into narrative form to see how the puzzle pieces fit together" (Saldaña, 2016, p. 277)—helped develop conclusions about the impact of the interventions.

Another form of data gathered was the sportsmanship ratings each team received from the referees. Sportsmanship ratings were completed for each team for each sport in the fall intramural program. These aggregated ratings were averaged for the four leagues completed: recreational soccer, competitive soccer, flag football, and volleyball. The average ratings were compared with the average ratings from the previous year to provide additional evaluative data.

When trying to transfer study findings, qualitative research benefits from triangulation. Triangulation includes "protocols or procedures which researchers and readers alike come to expect, efforts that go beyond simple repetition of data gathering to deliberative effort to find the validity of the data observed" (Stake, 1995, p. 109). The 
case study method used lends itself to triangulation as it "tends to involve in-depth and often longitudinal observation, in-depth interviewing, and document collection and analysis" (Glesne, 2016, p. 289). The study employed each of these methods: (a) in-depth observation of student staff and participants, (b) completion of a qualitative survey, (c) in-depth semi-structured interviews, and (d) an analysis of sportsmanship ratings.

\section{Participant and Data Care}

Kaiser's (2009) dominant approach provided a framework for how this study addressed confidentiality and privacy. The dominant approach addresses confidentiality during research planning and then at "three different points during the research phase: data collection, data cleaning, and dissemination of research results" (Kaiser, 2009, p. $1,634)$.

Data collection was ongoing during the study period through observations, field notes, and analytic notes. Survey collection took place after the fifth week of games. Student staff and players received an online survey that included an informed consent form (see Appendix F). The informed consent forms were appropriately documented and measures were taken to protect the security of all personal information including locking all research material behind two locks when they were not being used. One important modification to the typical consent form was disclosing my dissemination plans as part of the informed consent (Kaiser, 2009). This small gesture helped create a stronger sense of the purpose for the study and helped build trust with the participants.

The raw qualitative data gathered was kept confidential and any personal identifiers were removed and destroyed as soon as possible. As part of the data cleaning, confidentially was addressed by using pseudonyms for any written report. I stored the 
identifiers in a different location once the data was coded. Finally, in the dissemination of the study results, I ensured that the selected quotes and examples reduced the opportunity for deductive disclosure from the audience for whom the study is intended. The study data will be kept for at least five years and then shredded and recycled. Any research stored on a computer will be erased after five years.

\section{Role of the Researcher}

Glesne's (2016) writing on backyard research informed my role as researcher. While identifying that backyard research is attractive because there is "relatively easy access; the groundwork for rapport is already established; the research would be useful . . . for professional or personal life; and the amount of time and money needed for various research steps would be reduced" (p. 48), Glesne (2016) suggested caution for novice researchers like myself. Glesne also noted that data collection may be constrained due to participants' previous experience with the researcher; access and scheduling can get difficult if other job responsibilities pull the researcher away from the research; the uncovering of dangerous knowledge (information that is politically risky to share or hold) may occur; and closure of the research may be more difficult. Knowing these cautions allowed me to be aware of these challenges and address them.

As a professional in the world of collegiate recreation for 15 years, I brought an understanding of the role that sports play in students' lives, I understood where the intramural program fits in the overall structure of the university and the department, and I knew the baseline expectations from the NIRSA competency standards (NIRSA, 2015). I also approached the research with experience as a referee, a coach, and a participant in the sport I was researching. I was familiar with the rules and the regulations of the sport. 
Because I have such a lengthy and deep relationship to the subject, I carefully screened any preconceived ideas I might have had about participant experiences through discussions with student staff.

As the supervisor of the department in which this case study took place, I exercised caution about the power I wielded and its potential impact on the quality and candor of answers I received from the participants in the study. In addressing these concerns, I resonated with Glesne's (2016) request that, as researchers, "we interact with openness, honesty, and respect, not with the walls of professional distancing" (p. 144). While I was not "friends" with the student staff, as both a supervisor and teacher I managed the concurrent roles carefully and with an open attitude of partnership.

The multiple roles I had in this backyard research added risks to the study, including increased intimacy between the researcher, the researcher's colleagues, and the participants. Busier et al. (1997) defined intimacy as "relationships that include qualities of mutual care and friendship as well as revelation of, and respect for, personal vulnerabilities" (p. 165), and questioned, "If intimacy is a route to understanding, what should we, as researchers, consider as we engage intimately in relationships as part of our research?” (p. 166). In answer, Busier et al. identified four areas for the researcher to acknowledge: (a) relational reflexivity, (b) power in relation, (c) relational ethics, and (d) temporary understandings. Responding to each of these areas reduced the difficulties I faced in undertaking backyard research.

Addressing relational reflexivity, or how the relationship between researcher and researched is expressed, required "illuminating the interplay between researcher and researched ... to understand how the research relationship influences fieldwork and 
interpretation" (Busier et al., 1997, p. 167). While in this study there appeared to be minimal danger in intimacy between the researcher and the researched, because of the backyard research design, I created clarity on the role of the relationship between me and the student staff by reviewing my roles with the student staff, setting boundaries for my behavior, and providing the student staff information about my advisor and supervisor in case they wanted to ask questions or report anything that seemed out of the ordinary. The second concern of Busier et al. (1997) is the power relationships at play in intimacy. The utilization of community action research principles helped minimize the role that power played as the student staff actively participated in creating the interventions. The use of a colleague to conduct the in-depth interviews of the student staff additionally minimized the role of the power relationship.

The third concern—relational ethics—required me to "root our research intimacies in an ethic of care in which we remain at least partially responsible for the moral development of ourselves and of each person we encounter" (Busier et al., 1997, p. 168). To do this, Busier et al. (1997) maintained that we need collaboration, avoidance of imposition, and confirmation. The partnerships and trust built through use of community action research was essential in modeling an ethic of care. Finally, the concept of temporary understandings reveals that "relationships and degrees of intimacy change over time. A single 'understanding' never captures the relationship. Instead, all understandings are temporary constructions" (Busier et al., 1997, p. 168). In a bounded case study, temporary understandings have a good chance of emerging. I addressed this concern by 
allowing for six weeks between the conclusion of the case study and the drawing of conclusions.

\section{Summary of Methods}

Through a case study of an 8-week intramural soccer season, I explored how certain interventions impacted students' perceptions of their global-self. Using tenets of community action research, I engaged study participants in their own learning and addressed concerns about backyard research. The case study involved observations, field and analytic notes, open-ended surveys, in-depth interviews, and a review of sportsmanship data. The qualitative data analysis relied on coding survey and interview responses to assist in developing themes. Multiple steps taken in the data analysis protected the information and the confidentiality of survey and interview participants. 


\section{CHAPTER 4: FINDINGS}

The following chapter is a presentation of the findings. The findings were collected in four different stages of the study. As discussed in Chapter 3, observations were made starting with the pre-season meetings with the intramural student staff and continued until the end of the intramural season, an online survey was completed as the season concluded, in-depth interviews were completed after the season finished, and evaluative scores derived from team sportsmanship ratings were reviewed. The themes identified in the following chapter relate to the perceived change participants reported and the impact participation in intramural sports had on those perceptions. Killick's (2015) global-self acquisition relies on students developing numerous personal attributes through their lived experience. This chapter reviews the data found through the case study and arranges the data into two subsections to answer the research questions. The first section helps to answer the first research question by identifying six themes related to attributes of the global-self. The second section reviews the evaluative data to help answer the second research question. Because of the nature of this case study data, there is overlap between the identification of themes and the evaluative data.

\section{Themes}

Themes are a common way to organize research gathered through qualitative methods (Saldaña, 2016). Themes emerge through the coding of the data and are helpful in describing the outcomes of the study. Eclectic coding created broad based ideas that were the initial step in defining the identified themes. With these results in hand, causation coding helped me identify reasons "why" the broad themes emerged. Axial coding then cleaned up the data so that it was ready to be placed into more clearly 
defined themes, and code weaving brought data together in a coherent way to identify the final themes: (a) communication, (b) stereotyping, (c) humanizing, (d) teamwork, (e) community, and (f) self-awareness.

\section{Communication}

The theme of communication emerged from the data and occurred throughout the study in many ways. Communication occurred between players of the same team, between competing teams, and between participants and the student staff. Both verbal and nonverbal communication occurred throughout the course of the study. Improved communication was a theme that ran through all facets of the study and is an important component in resolving conflict among teammates, as suggested by Mishra, Sharma, and Kamalanabhan (2016):

Effective communication is apparent when team members listen to one another and attempt to build on each other's contributions. In team situations, where disagreement is likely to occur, the ability to recognize and resolve conflict is important to the team's success. (p. 215)

It was clear through the observations that communication on the soccer field manifested in diverse ways. One of the clearest observations was that there was a difference in the way that recreational and competitive soccer teams communicated. During recreational league games, communication between students was mostly positive and there were limited arguments between players or between players and student staff. In the recreational league, the largest disagreements were between student staff members who had dual roles as referees and players. In one case, a student staff referee who was playing became so frustrated with another student staff referee who was refereeing that he chose to take himself out of a game to cool down. Nonverbally, the player appeared 
very upset, and during the game, he confided in me his frustrations with his fellow student staff. The student staff/player did not lose his composure, but instead talked through his frustrations with me.

During the season, it was clear that communication between students on the same team was positive at the recreational level. One team in the recreational league went so far as to get a volunteer coach to help improve their communication. Quang, who played in the recreational leagues, supported this observation in his interview reporting that he "learned that once [he was] a part of an established community [e.g., a soccer team] then direct and open communication [was] the fastest way to get stuff done." There were no observations of violence or inappropriate behavior during the recreational league games.

In comparison, competitive teams' communication demonstrated a broader range of patterns and outcomes. While some teams were kind and supportive to each other, other teams were aggressive with their own teammates, indicated by the observation of multiple instances of scolding and finger-waving. Communication styles appeared to follow a regional trend. Teams from two Middle Eastern countries - Kuwait and Saudi Arabia - were noticeably more vocal with each other than teams that were more mixed or from more Western cultures. It appeared through tone of voice, body language, and gestures that the Middle Eastern teams communicated more, and on a different octave, than their non-Middle Eastern colleagues. The octaves were both negative (disagreements, frustration, apparent blame) and positive (celebratory, playful, supportive). Sam who was a senior, biracial, male student from the United States who played in the competitive league on a team of mostly Middle Eastern students from Afghanistan, Saudi Arabia, and Turkey spoke to this difference in his interview 
remarking, "I can't think of a halftime where someone didn't come off the field and yell at someone about something." In some cultures, that style of communication might be problematic, but Sam went on to explain that it "solved that issue much quicker and [they] were able to get on to actually solving a core issue or trying to figure out what it was about [their] defense or why [their] offense couldn't score." Communication between students of different cultures revealed different responses depending on the skills or attitudes of the student. Some students had a difficult time communicating, which led to falling back on stereotypes as Sam addressed:

I know I can tell by tone of voice, body language and things, I can kind of interact with them. It's not so much a barrier, but when I see our players speaking with other players or the other team speaking, when you have no idea who that person is and have no idea how they carry about a conversation or anything like that, when you can't understand a single word that they're actually saying, it is hard. Then you rely on just whatever generalizations that you have. I think that that's when languages really present a barrier. When you're not able to understand the other person, then it's hard to get over those stereotypes and generalizations that you might form because you're not learning anything about them.

Of the in-depth interviews, Sam reflected the most on the role of communication in his experience of the intramural soccer league. Growing up in a mid-sized, rural town in the Northwest, Sam's prior life experience influenced his depth of understanding. Sam went to a school that had a "30 or 40 percent Latino population" and had lots of friends from "different backgrounds . . . socioeconomically," which gave him "a lot of perspective about really being able to view someone as another person." Playing in the competitive league, he observed that more international students surrounded him; in fact, Sam was the only Anglo student on his team of mostly Middle Eastern students. Noting that there were North African, Japanese, and other teams of different nationalities, he reported: 
Most of the teams, when ... they get scored on . . . everyone spreads blame the same way. Maybe it's not the most humanistic or benevolent quality, but it's something that's a similarity among most of the cultural groups . . . it kind of gave you the same effect [that] "they have the same problems we have."

Sam linked this observation to his own improved communication skills: "We had

a lot more interactions that were more productive than just laying the blame on someone .

. . we solved issue[s] quicker." An online survey response reinforced Sam's observations:

[A] good sense of nonverbal communication can help in getting on the same page with teammates. Giving verbal praise for good/hard work on the field can also help improve comfort with teammates where there is some kind of cultural barrier or difference.

Alexis, who is bilingual and Latina, yet who presents as White, had a different experience based on her communication. Due to being bilingual, Alexis deepened her interaction with other Latina/o players and created a rapport on the field that lowered the intensity of the games. She noted in her interview:

It's funny because some of the other teams who visibly are Hispanic or Brown . . . I don't know how they identify, but we speak in Spanish and we're like, "We know what you're saying, don't try to trick us." So, that was kind of fun.

Language skills, both having them and not having them, correlated to students' ability to know the other students against whom they played. Edward, for instance, in his in-depth interview, spoke directly about how he had learned that communication across cultures required more understanding that just communicating with others who spoke the same language:

We're all different and therefore we're all going to talk about and think about things different, so we all need to have this understanding that we're not going to get it right on the first try when trying to cross cultures. We're trying to just communicate in between cultures and so to take a step back and try and slow it down and break it down in terms of communicating and trying to understand each other. 
Similarly, another student addressed his fear of a lack of communication due to cultural differences. He shared, "I played with a team from mainly India. From the start, I was worried we may not communicate, but as the season rolled along, we got more comfortable with each other and [got] along well as a team." An international student echoed this sentiment by relating, "Being a foreigner myself, I am in the unique position to experience [being equally human]. [I] learned to be careful how to express myself when talking to others from different cultures, so I do not get misunderstood." The data provided by these students highlight that the interactions between international and domestic students is an important nexus for learning the value of good communication.

The ability to communicate is one of the core aspects of the ability to act-in-theworld and the data observed and reported suggests its importance was not lost on the players and student staff. In fact, communication was an essential part of students' ability to address stereotyping - another theme that emerged in the study.

\section{Stereotyping}

Stereotyping is a natural process humans use to help negotiate the immense information that occurs in our cognitive functioning. Our brains naturally try to simplify and categorize information for efficiency. While stereotyping assists our cognitive efficiency, stereotyping is also a "threat to intercultural interactions" (Killick, 2015, p. 91) because it negatively impacts "our capabilities to learn from others or adopt 'objective' stances towards the behaviours, beliefs, or aspirations of others” (p. 91). The development of the global-self requires that we realize that each person has an equal right to being human, and the practice of stereotyping can diminish the importance of this attribute. Killick (2015) warns that 
when a peer who is not identifiably "other" fails to contribute fully to a group work task the fault is likely to be attributed to the individual, but when an international student does so, the fault is often attributed to her national or even her generic international-student identity. (p. 161)

Knowing that stereotyping was a natural human instinct, the intervention primed students to consciously think about the concept by using a pre-game question during the fourth week to ask students directly if they had ever been stereotyped.

Two views emerged when students were asked during the pre-game introduction if they had ever been stereotyped. First, students reported that they were often stereotyped based on their external personal attributes. One student said that being from San Francisco made people wonder if he was gay; another student voiced that because she was French, people assumed she was arrogant. During one pre-game meeting between teams that were mostly international students, the question whether they had ever been stereotyped was, literally, laughable. Many of the players recognized it as selfevident, that they, as international students, would have experienced stereotypes. Second, students reflected on the inability of people to fit nicely in boxes. One biracial student noted, 'I'm either Spanish or not ... I'm either never enough Spanish or never enough American." A Ukrainian student described that not only did people think he was Russian, but, despite his own beliefs towards sexual orientation, a typical reaction to him was that any gay people should be afraid of him due to his nationality. One of the survey responses identified a similar frustration with negative stereotyping: "I am one of only two Caucasian players on my team and interactions have been great. I do notice that there are some race-related issues, mostly with players stereotyping other players." 
While some students could identify stereotypes and share their experiences of being stereotyped, other students were still learning how to address their stereotypes of others. For example, there was a student employee from India who was both a talented soccer player and a talented referee. One employee reported about his relationship with this student by stating, "He used soccer to get to know one of our new staff members from India to relate his and my life experiences even though we grew up in very different places." Yet, because he was Indian, many participants engaged in conversations with him during the season about how he challenged their perception of Indian athletes since he did not play cricket, thus inadvertently reinforcing stereotypes.

Stereotypes based on gender were observed and reported as challenging. It was clear from watching the games that female referees were questioned more than male referees. The observations did not uncover a theme of more gender stereotyping emanating from international or domestic students. In fact, the most heated interaction involving a female referee involved a non-player White male - a father of one of the players. One female referee related in the survey: "Players that come from different backgrounds than myself have been somewhat hard to interact with. I think that being a female ref does not help the cause." Conversely, one of the female players reported: "Being female while most of the league was guys was interesting. It didn't seem that anyone really cared about differences once we were on the field. We were there to play."

Edward, a student staff person, reported that he had also been stereotyped.

Edward noted in his interview that the rules in intramurals don't always lead to stereotyping, but . . . as a ref, I've gotten a lot of flak for being an American ref, so [people assume] "Americans don't know soccer as well as the 
rest of the world" and ... we're enforcing this rule that the rest of the world doesn't play so it's an "American” thing.

Stereotyping appeared to not be just a one-way issue where domestic students stereotyped international students. Many international students also expressed surprise to me that Americans even knew how to play soccer, as they thought that all Americans only played and supported American football.

Despite some of these negative findings, students were still able to differentiate their opinions about a person based on the different contexts in which they interacted. In short, students did not attribute a person's whole personality to what they saw them do on the field. As identified in Chapter 2, stereotyping of varsity athletes often carries negative connotations over to off-the-field activities. In this study, however, many of the intramural players could separate a player's behavior on the field from their behavior off the field. This was a common survey response and is best exemplified by this response: "The biggest thing I've learned is that the person you see on the field and the person they are off the field are often two different people."

The topic of stereotypes came up strongly in many of the in-depth interviews. Helen was a self-identified White woman from the Midwest who had moved away from home to some place new because she realized she needed to broaden her worldview. In her interview, she reported that her own biases changed because of her participation in intramural soccer. During the interview, Helen described a new recognition of her responsibility to counter stereotypes:

When I call home and I talk to my family from back home, I'm like, "You can't say that," and they're like "Why?" and I was like, "Because I've experienced . . . it's not like that . . . like you can't do that." And they were like, "What? No." And 
I'm like, "Yes, it's different . . that's not how they are . . that's not what it means. You're just wrong. You need to experience it first."

Alexis had a similar experience with her teammates, noting how the pre-game introductions impacted her view of stereotypes: "Well, a lot of the European countries, really being able to talk to people from there. And there's a lot of stigma and stereotypes that we're fed growing up . . . and it was like, 'Oh wait, that's not true at all."' Helen and Alexis both experienced something new in their self-development resulting from their participation and were able to clearly explain a more refined understanding of stereotypes.

Quang, a Vietnamese graduate student, also had a lot to say about stereotypes. Early in his interview, Quang was stereotyping people. But, as he spoke more about his actual interactions with people from other countries, he realized that his initial stereotypes of other people were modified by playing with people from other countries:

For example, Europeans, they only pass to each other. They don't like to pass to the other American or Asian. They only pass to each other. Sometimes they pass a ball all the way to the other goal and then they didn't attack. They justify it's for fun. That may be the time when I don't trust other Europeans, because they tend to pass to each other. They live in a small community where they play with each other then they like to pass to each other. They didn't pass to other guys. So maybe, it depends on the guys and how I view him. For example, Japanese they are very good and they pass to each other and they pass to other guys. Americans, you guys pass to each other and you don't seem to be really aggressive if someone didn't perform the way you expect. So, it's actually depends. The race may give you a first impression, but not everyone. I met some European guys who actually pass to the other guys.

Quang started his response by stereotyping people based on extremely large categories: Europeans, American, or Asian. As he answered the question, he became more aware that there are times when "it depends," and that his first impression may not 
always end up being the correct one. Quang had a similar change in his view of Brazilian students against whom he played:

For example, I see some Brazil guys and I assume he must be really good. But it turned out not every Brazilian is good at soccer. So, it turned out the guy didn't play very well. So, I just acknowledged that . . . Brazilian that made a bad impression on me ... doesn't mean that the other one will be bad like him.

Stereotyping is a complex human behavior, and during the study it occurred in many ways for students. Students were observed positively discussing their relationship to being stereotyped, and they reported that they had experienced negative stereotypes. Stereotyping is a significant barrier to humanizing the other, another theme the study discovered.

\section{Humanizing}

Humanizing was a clear theme that emerged through the analysis of the survey results and the in-depth interviews. Reducing the prevalence of group identity is one of Killick's (2015) means to creating conditions for the development of the global-self. Killick's key to creating this successful condition is when people recognize that others with whom they interact are equally human. This concept was taught at the student staff training and at the captain's meeting, but it was not explicitly talked about in the pre- or post-game interventions. Unlike the themes discussed so far, this attribute was not observed; rather, it was coded from the answers to the survey and in-depth interviews.

The online survey included the following prompt to participants: "Describe any new insights you had about the idea that the others participating and working in the league are equally human." Nearly one third of the respondents believed they already knew that others were equally human, exemplified by this survey response: "I have 
always been aware that all people are equally human, and this experience simply reinforced this idea." Conversely, other survey results indicated that one of the main reasons students felt a connection to others was simply because they were playing the same sport, illustrated by this straightforward survey response: "Everybody was there to play soccer, nobody was different because of their backgrounds.”

An unexpected outcome of the data was the humanizing of the student staff, described as happening because "the staff became more of a real concept after actually interacting with them. Before that, they were just a vague idea." Though this may oversimplify the complexities involved in humanizing, it speaks to the ability to find common ground in sports. Students acknowledged that they had been made aware of the concept of equality through their interaction with the intramural program student staff:

You can feel and see the efforts laid down by the intramurals staff to have us be respectful to one another. I understand that racial and ethnic issues are a big problem in the world, and being myself a foreigner to this land, I salute the efforts by the people in the intramurals staff who are actually working toward helping us learn about another and emphasizing the idea that we are all equal.

Similarly, another student reported: "Seeing the refs also playing on other teams reinforced in me the idea that all players on the field are equally human and have their own lives and backgrounds," while another student related, "I felt like it was a more friendly game and I saw the staff as people instead of just a uniform of position." The data suggest that not all students were supportive of the student staff playing. There were a handful of comments about the student staff playing in games, summarized well by this response: "For the staff, I do not really appreciate when a ref plays on the opponent team, even if the refs are trying to be equal. It is hard to believe/see sometimes." Overall, 
however, the data suggest that student staff interacting with participants helped to humanize them and potentially assisted in the reduction of conflict.

Helen, from the Midwest, noted how her interactions, through the pre-game introductions and playing with other students, transferred her humanizing to venues beyond intramurals:

I think [intramural soccer] breaks down, for me, all the barriers. You stop seeing all the differences and you kind of become one, especially when you're forced to play with them and then even playing against them. ... Yeah, they're competitive, but you see them in a different light and view than you may see them on the street. It really breaks down all, everything, and just kind of allows you to bond over the sport.

The pre-game activities were designed to support the humanization of the other, and two survey responses provide evidence of this humanization. One student responded:

I thought [the pre-game introductions were] weird, but as I played more games, I appreciated [it] because you didn't just see the opponents, or refs, as enemies, but as people. You were more able to empathize with people - they weren't just the other team but fellow students.

Another student shared:

I connected with [other players] before the game in those pre-game meet and greets. I thought of them as acquaintances/friends rather than opponents I want to beat. It showed the funny side of some people, which was great because when the game starts, they might not show that as much.

Harry who was a Ph.D. student from the United States revealed:

There's, like, the kind of community aspect where, as you get to know more people, you know your circle widens a little bit. There's kind of the social circle aspect where you can get, like, a more friendly initial disposition toward the opposing side.

The survey responses indicated some movement toward positive humanization, but there remains a nuanced line in sports between acknowledging that other people are 
equally human and group identification when conflict arises. Despite literature

acknowledging that sports can create a perception that one team or one person is better than the other (Kerr \& Males, 2010; Wann \& Grieve, 2005), that result was not reported in this study. A survey response revealed this nuance by encouraging that differences should be acknowledged "without malice":

Everybody should behave on the field, and everybody should know everyone on the field is a human being with their own personality and history, but part of the sport involves emotions, and players should understand that emotions can and should be expressed verbally and physically, but without malice.

One student had this realization to share: "Working with people from different backgrounds than me is the same as working with people who are similar to me." An interesting extension of this finding is that while the initial focus of the study was on players learning about the other team in an attempt to humanize them, the results indicated that while learning about the other team did happen, the interventions resulted in players knowing their own team better.

\section{Teamwork}

Teamwork, the next theme identified, is often an assumed outcome of playing team sports, and that it is an inherently good outcome often goes without question. Successful teamwork helps people move toward collective work and away from individual identity. Mishra et al. (2016) wrote, "The creation of feelings of trust, respect, and understanding are key elements in team development" (p. 215). Successful teamwork improves the ability to work with others and is linked to social identity theory by its connection to how individuals fit into larger groups because of a shared experience on a team. Individuals may create an identity based on their affiliation with a team. 
Throughout the intramural soccer season, teamwork was observed as a feature of the shared experiences and students discussed the impact of teamwork on them in their survey results and in-depth interviews.

Teamwork relies on mutual performance monitoring, which is the ability to have a shared understanding to help facilitate self-correction of behavior. Quang identified this concept in his description of his reactions to player behavior: "You should not blame it on one or two players ... this is the responsibility for the entire team. They should stay strong together." Adjusting to somebody else's way of being through teamwork, identified as adaptability (Salas et al., 2005), emerged in the survey data. One student replied, "My team had never played together before so we had to work hard to adjust to each other's playing styles. In retrospect, I also learned to have a positive attitude/good sportsmanship/perseverance," while another student replied, "I've learned that everyone thinks and behaves in different ways and that I had to adapt to those people."

Harry noted a different outcome of teamwork — social normalizing — and its impact on the pre-game intervention:

I notice a lot of times, even with a lot of people on my team, there's a tendency to kind of socially normalize. People, when they're asked a question about, "Oh, what is your favorite place?" or these kind of more open-ended feeling questions, they want to say things that just normalize with, "Oh, I'm thankful for my friends."

Harry's feedback on the role of the pre-game intervention highlights an important role of teamwork - a responsibility to have a team orientation. Having a team orientation emerged frequently in the survey responses. For example, one student replied in the survey that their participation helped them to "learn to communicate, be patient, control [their] anger, encourage weaker teammates, [and to not] get mad at them," while another 
survey response said that they needed to work on their skills to "improve interactions with [their] teammates."

Survey responses also indicated that students learned from each other and developed a sense of gratitude through their teamwork. Responses supporting this idea included one student who conveyed that they "do [their] best to get every teammate involved in the game so that [they] enjoy equally," while another student explained, "I just appreciated the fact that every team put in the effort every game to make things run smoothly." Having teamwork appears to have been an important attribute that players appreciated, summed up by this quote:

I know very well that we can all work for a common goal regardless of our backgrounds and soccer is a great example of this. While we all have different attributes and backgrounds, this sport brings us together so that we may better ourselves.

Successful teamwork requires trust, shared understanding, adaptability, and an

orientation toward relying on others for shared success. These attributes associated with teamwork that are developed through sports can influence student learning and the development of the global-self. They are also important attributes to successful communities, the next theme found in the data.

\section{Communities}

The formation of communities, a theme important to the development of the global-self, is a tool often used in the classroom. Killick (2015) notes that "it is group work, particularly when it is directly linked to assessment, which often causes students to voice their concerns about working across cultures" (p. 161). Intramurals are essentially small communities (teams) competing together while another community (staff) is responsible for facilitation of the activities. The intersection of these different 
communities created many opportunities for students to learn, as evidenced by the observations and assessment responses. Both the student staff and the players were clear in the impact that their participation had on their sense that they developed strong communities through their participation. The data presented below provides insight into student perceptions on the importance of communities to developing attributes of the global-self.

The first sign of community building occurred during the student staff training. With the delay in the start of the season, the student staff's energy was low when they started the training module on the development of the global-self. However, as the training unfolded and the material became relevant to their roles, students began to lean in. The questions about their global-self resulted in robust conversation. When the students watched the videos on soccer derbies, a visible shift occurred in the camaraderie between student staff, and a sense of common purpose and perspective appeared.

The role of community in players' perception of their global-self was clear in the findings from the online survey. These perceptions fell in to two different relationships with community. On one hand, player survey responses indicated that their overall sense of community grew through their participation; one student noted simply that they had a "greater sense of community after [their] interactions," while another said that their "sense of community grew." On the other hand, students reported the positive outcomes associated with better communities, including "direct and open communication," "uniting together," and that "education, sport, and community are important to us all." These broad impacts of community were found during the in-depth interviews. 
Ned, a male, Chinese, international undergraduate student who played in the recreation league stood out for his observations about the impact the intramurals community had on him and others. As a team captain, Ned's engagement was more indepth than the regular players, and he noted the benefits of the captains' meeting: "[It's] good for us to know each other, and we have [to] kind of like just know each other and kind of know who are we playing with and what kind of people they are." This quote highlights the importance of knowing what kind of people you are interacting with, and for Ned, the captains' meeting provided a powerful opportunity to build community. Later in his interview, Ned noted that the pre-game activities helped him feel as if he was in community with others:

You can know each other before the game and ... playing more like smooth, I mean, like, kind of have a relation with them and it's better for the game. And I think it's more good to make friends after the game, yeah, it's helpful.

Ned's remarks recognize that his participation in intramural sports helped him create community: "We make teammates and we have to work together and to win the game, right, and so we become friends after the game." Ned did not just make friends with those like him; he was part of a mixed team of players of multiple nationalities and ages, the exact scenario Killick proposes leads students to develop their self-in-the-world.

Like Ned, Helen spoke about the positive impact of community on her learning:

I think that it ties people from different countries, different areas, and it gives something in common that's easy to talk about. And that really breaks down everything. And no matter where you're from it kind of connects you and allows you to be able to talk about stuff and be able to really get to know someone some.

Helen identified that her participation allowed her to "be able to talk about stuff," but she qualified her ability to get to know somebody with the word "some." Helen, like others, 
did not necessarily think that the participation in the pre-game activities resulted in indepth knowledge of other people, but recognized that the activities had provided her an opportunity to make progress on getting to know somebody. While the pre-game activities may have created the space for future community, in and of themselves they did not create communities. Instead, the pre-game activities appear to have served as an introduction to further exploration of people's assumptions and attitudes toward each other. Helen expressed that the pre-game activity helped her learn about her own team as much as the other team. This was an unintended outcome but a pretty clear one for her: “That icebreaker made everything a lot easier ... I didn't know my team before I got put on it. So definitely ... was ... like we're going to throw you right [into] asking questions and get a deeper level with the people you're going to be out there playing with." The interventions, to her apparent surprise, appeared to have helped Helen form stronger communities.

Similarly, Amine, a North African graduate student, addressed the value of learning about other cultures by participating in a community:

By playing with them and by playing with South Americans, you start to kind of understand ... you start to learn a little more about them. So, you start to learn about different cultures . . . one of the reasons I came to the U.S. is because I was interested in learning about other people's cultures.

Amine was one of the participants who stood out in the league. He was joyful in his interactions with others, and while watching him play, it was clear that he had developed an appropriate knowledge of the context of the moment. When he played against people who were clearly not as good as he was, he noticeably lowered his intensity and worked hard to support others. In a similar vein, when he was in a more competitive match, he 
demonstrated the ability to support others while simultaneously ratcheting up his intensity. Amine demonstrated that his global-self was developing as he actively sought out players of all nationalities, eventually spending most of his time with a couple of German players and interacting well with the numerous American players.

Communication, creating similarities, and uniting are positive attributes of being in community. By growing communities stronger through the intentional interventions, students reported these attributes that are essential to the global-self and demonstrated growth in their self-awareness.

\section{Self-Awareness}

At the core of Killick's (2015) self-in-the-world is the important component of students' self-knowledge that they need to be ready to "have an equally skeptical stance towards [their] own way of being as [they] have towards others" (p. 31). Being aware of your own way of being is essential to developing self-awareness. Self-awareness first emerged as a theme in the survey results and then in more detail in the in-depth interviews.

The ability to maintain composure is an indicator of self-awareness (Ruderman, Hannum, Leslie, \& Steed, 2001) and multiple students reported the development of this attribute in the survey results. For instance, one student said, "I had to acknowledge my space and find a balance between being respectful and competitive," and another noted they "kind of brushed things off a lot easier." In more colloquial terms, one student noted, "I was going out of my way to make sure that they weren't pissed at me, basically, so it just took the edge off from what I could tell." 
The finding of self-awareness surfaced in the in-depth interviews as well. Ned, a Chinese undergraduate student, exhibited early in his interview that his self-awareness was part of what made him have more appreciation for different cultures:

So, I'm different because I have a different culture . . . I'm from China and I kind of have a different perspective from other culture ... I want to learn the same things as others that are from a different culture, they have different perspective about the topic thing, and yeah, we can share our thoughts and create a better decision.

Ned's acknowledgement that he had a different perspective, but that it was important that he learn about a different culture to make better decisions, demonstrated a developing self-awareness. Quang, a senior, male, computer science major from South Asia who played in the recreation league also recognized that self-awareness is a requirement to both understand our place in the world and to display empathy to others:

You are aware of your teammates, and when they fall down you know that you stop the ball and try to figure out if he got injured or not, so you are more selfaware that you are just an individual in the global world. You are not the sun and the entire galaxy. I think it helps us to care about each other and care about how other people think and feel. When you decide you're going to say something you think before, "Would that hurt the guy? Will that impact the other team members?"

When he recognized that he was "not the sun and the entire galaxy," Quang placed his self-awareness in a larger context, demonstrating that he understood his larger responsibility to others. Helen noticed similar changes in her self-awareness during her interview:

Having the experience with people who are different than you and seeing that they're . . . different, but you're realistically . . . the exact same, almost, you just do things a little differently, you understand things a little differently, you talk a little differently, but when it gets down to it ... the root is the same 
Because of her shared experience with others in the intramural league, Helen concluded that "the root is the same," demonstrating that while she might be aware of some differences between people, at the end of the day, the similarities are more powerful. Helen's quote suggests that she was developing an "equally skeptical stance towards [her] own way of being" (Killick, 2015) and that the study may have helped her on this path. Self-awareness is hard to demonstrate and to measure. The data that emerged from the study suggests that it is an attribute that participants were aware of and improved.

Six themes emerged from the data that related to the research question. These themes included: (a) communication, (b) stereotyping, (c) humanizing, (d) teamwork, (e) community, and (f) self-awareness. Combined, the data informing these six themes provide support for the argument that the study was successful in helping students move toward their global-self. The next section highlights data related to how students experienced the actual intramural program.

\section{Evaluative Review}

The experiences students had of the actual interventions during the intramural season helped inform the answer to the second research question. To inform future practical implications for practitioners in intramurals, a synopsis of survey responses is presented below. This study introduced a new method for implementing intramural sports, and one of the players summed up my observations of the student experience when she explained that she

felt that the changes of having individuals come together and share things made the actual game friendlier. I found that people enjoyed playing a little more, don't 
get me wrong people still want to win, but they are caring about others well-being at the same time.

This section reviews the evaluative comments from the survey and the in-depth interviews to assist in the assessment of the overall quality of the student experience.

Overall, the data indicated that students enjoyed their experiences during the intramural season. Additional data reviewed after this section suggests that students improved their sportsmanship, indicating less conflict between players and between players and student staff.

\section{Pre- and Post-Game Interventions}

The data suggested that the pre-game activities were an effective intervention.

Some students reported that the interventions were strange at first, represented by a player who reported that "the questions really didn't make sense and didn't make that much of difference to get to know each other." But this was not the predominant attitude, as demonstrated in these two responses:

It was important to know where the other players come from so you get a little background of their experience in soccer and of their experience in the world. A lot of players come from other states and countries, so seeing them get involved is pretty awesome.

The changes of having individuals come together and share things made the actual game friendlier. I found that people enjoyed playing a little more. Don't get me wrong, people still want to win, but they are caring about others well-being at the same time.

The impact that occurred on the student staff members was magnified as a result

of student staff's multiple interactions with multiple teams. Nesto pointed out:

The biggest impact was the physical implementation of introductions before the game and getting to know people before every single game for six to seven hours a night ... so it was very easy for refs to create a nice relationship with anybody on the team. 
The post-game interventions did not have similar participation rates as the pregame interventions. Students who participated offered many ideas to improve this intervention, including simplifying the questions, simply offering a post-game activity, and improving the communication about the activity. Despite these suggestions, respondents still provided positive reviews. One student shared, "Overall . . . I enjoy[ed] these "strategies." They were refreshing. Another student responded:

Everybody loves candy and pizza so that game was a great hit. I found myself working with multiple people to find out the answers to the questions and in the process I learned some things that I thought I actually knew but I was wrong.

Table 3 summarizes the qualitative responses students provided when asked specifically about their reactions to the pre- and post-game interventions. Total survey response demographics are presented in Appendix J.

Table 3

Player and Employee Response to Pre- and Post-Game Interventions

\begin{tabular}{|c|c|c|c|}
\hline & Positive & Negative & Neutral \\
\hline Players & 34 & 5 & 5 \\
\hline Student Staff & 3 & 2 & 0 \\
\hline Total & 37 & 7 & 5 \\
\hline
\end{tabular}

The data indicate a strong positive reaction to the interventions that took place during the intramural games. Seventy-seven percent of the survey responses indicated that players seemed to have a positive reaction to the pre- and post-game interventions. Student employees were less certain about the value of the interventions, as only $60 \%$ claimed a positive interaction. Together, $76 \%$ of all participants surveyed had positive reactions to the pre- and post-game interventions. 


\section{Change in Interactions}

When survey respondents were asked if their participation in intramural sports changed how they interacted with other players or student staff, 37 of the 47 responses (79\%) acknowledged their participation had changed their views. Of these 37 responses, not one response indicated a change for the worse. The changes students observed in themselves ranged from increased respect to better understanding of the student staff to an overall increase in humanization. Among the changes that students reported were that they "felt more welcomed with [their] team that was mostly from another country, and it makes [them] realize how easy it [was] to talk to people sometimes when [they] need a friend," and they "got more comfortable, not only with [their] teammates, but with the staff and [their] opponents." When a student did not feel their interactions with others had changed, it was often described similar to this survey response: "I gained a greater awareness of the diversity among IM players, but my interactions didn't change- they remained respectful and positive."

The issue of time also factored into some students' perceptions of the quality of the intramural season and its impact on the ability to change. One student reported, "At times, they were a little rushed due to time constraints, but I think it allowed both teams to laugh together and connect before competing," while other students expressed similar sentiments to a survey response that suggested that "the season was too short and the games too infrequent" for them to be given enough time to really change.

\section{Overall Experience}

When students were asked to describe their overall experience with the intramural season, the results were overwhelmingly positive. Of the 48 responses, 47 participants 
$(98 \%)$ had a positive overall view of their experience. The only response that was not positive was neutral: "This season I think, it was OK. It did not come close to the [seasons] we had 10 years ago, but it was without any major incidents, which is encouraging." The remaining replies ranged from simply, "Awesome," to explaining that they enjoyed the season because they "got to meet people and actually talk to them, which normally would not have happened on [their] own. It gave a sense of friendly competition rather than cut throat gladiator winning, which is not a great atmosphere to play in." When reviewing the word count from the responses to the survey question regarding their overall experience, once extraneous words were removed, the number one response was "fun," mentioned 18 times. The second most popular word in the summary was "great." The use of "great" was used to describe multiple types of enthusiasm, including that it was a "great way to relieve stress" and "great help for me to [get] accustomed to my new life in the U.S." The survey response data indicated that students had an overall positive experience.

\section{Sportsmanship}

A review of two years of sportsmanship standings helped shed light on the impact of the interventions on player sportsmanship. The summarized sportsmanship data in Table 4 indicates that the study interventions had a positive impact on the behavior of the intramural soccer players. 
Table 4

Sportsmanship Ratings for Intramural Sport Leagues from 2015 and 2016

\begin{tabular}{|c|c|c|c|c|}
\hline & \multicolumn{2}{|c|}{ Competitive Soccer League } & \multicolumn{2}{|c|}{ Recreation Soccer League } \\
\hline & $\begin{array}{c}\text { Number of } \\
\text { Teams }\end{array}$ & $\begin{array}{l}\text { Sportsmanship } \\
\text { Score }\end{array}$ & $\begin{array}{l}\text { Number of } \\
\text { Teams }\end{array}$ & $\begin{array}{c}\text { Sportsmanship } \\
\text { Score }\end{array}$ \\
\hline 2015 & 10 & 3.97 & 8 & 4.41 \\
\hline \multirow[t]{3}{*}{2016} & 9 & 4.29 & 12 & 4.58 \\
\hline & \multicolumn{2}{|c|}{ Flag Football League } & \multicolumn{2}{|c|}{ Volleyball League } \\
\hline & $\begin{array}{l}\text { Number of } \\
\text { Teams }\end{array}$ & $\begin{array}{c}\text { Sportsmanship } \\
\text { Score }\end{array}$ & $\begin{array}{l}\text { Number of } \\
\text { Teams }\end{array}$ & $\begin{array}{c}\text { Sportsmanship } \\
\text { Score }\end{array}$ \\
\hline 2015 & 9 & 4.47 & 6 & 4.87 \\
\hline 2016 & 9 & 4.33 & 6 & 4.76 \\
\hline
\end{tabular}

Referees give each team a sportsmanship ratings on a 1 to 5 scale at the completion of a game; a 5 is high and a 1 is low in this scale. A completed data review for the three team sports offered in Fall 2016 (flag football, volleyball, and soccer showed) showed that flag football sportsmanship ratings fell from 4.47 in 2015 to 4.43 in 2016, volleyball sportsmanship ratings fell from 4.87 to 4.76 , competitive soccer sportsmanship ratings increased from 3.97 to 4.29 , and recreational soccer sportsmanship ratings increased from 4.41 to 4.58 . While the data was not tested for statistical significance, it does suggest that the interventions conducted during the intramural season did have a positive impact on student behavior during the games. Improved student behavior creates a safer environment which boosts the opportunity for learning.

\section{Conclusion}

The results of the study were culled from five methods of assessment: observations, field notes, survey results, semi-structured interviews, and evaluative data. The themes that emerged from the study were communication, humanizing, stereotyping, 
community, teamwork, and self-awareness. The themes were described, placed in the context of the development of the global-self, and related back to the literature review.

The evaluative data review indicated that most study participants had a positive experience with the study interventions. The data was reviewed to look for how students reported their changes in interactions with others and their overall satisfaction with their experience during the intramural soccer season. Finally, a review of the sportsmanship data was performed to analyze if students reported any changes in their sportsmanship. 


\section{CHAPTER 5: DISCUSSION AND IMPLICATIONS}

The purpose of this study was to explore how a large, urban university's intramural sport program, when intentionally designed to modify how students perceive their global-self, impacted student's sense of their global-self and their overall perceptions of the designed program. The purpose of the study was twofold. The first intention of the study was to understand how students experience the development of their global-self through participation in a collegiate intramural soccer program. The second intention of the study was to understand students' experience of the program to effectively operationalize interventions in collegiate recreation programming that support the development of the global-self. Two research questions guided the study:

- How do students participating in a collegiate intramural soccer program perceive the development of their global-self after experiencing interventions intentionally designed to enhance discussion of, and reflection on, attributes of the global-self?

- How do students experience an intramural soccer program designed to encourage the development of global-self characteristics?

The case study was based on community action research and relied on partnerships between the researcher, the participants, and intramural program staff. Working together, the researcher and the student staff developed trainings that supported the development of the global-self for student staff and team captains. Interventions for pre- and post-intramural games were developed and subsequently modified with the intramural staff to align the interventions with the realities of game management. The 
interventions were implemented and assessed through observation, surveys, in-depth interviews, and evaluative data analysis.

The discussion and implications in the next section summarize the themes identified in the study and draw conclusions about the interventions effectiveness on helping students develop of the global-self. The interpretation of the results includes a comparison between the findings and the results of the literature review. The implications on the practice of collegiate recreation professionals are provided, limitations of the study are highlighted, and further research ideas are identified.

\section{Discussion}

Participation in sport can have both positive and negative influences on the development of the global-self. Sports, particularly as the level of competition rises, creates winners and losers, lowers moral reasoning and behavior (Doty, 2006), and can inspire negative personal traits such as "selfishness, violence, cheating, intemperance, and arrogance" (Sciarabba, 2012). On the other hand, sports can help develop the values, knowledge, and skills associated with the development of the global-self: building of community, humanizing, communicating, self-sacrifice, respect, friendship, and even the pursuit of excellence (Shields \& Bredemeier, 1995). The results of the study indicate that the designed intramural program created positive changes in students' development of their global-self. Most students reported a positive overall experience with the intramural program as it was designed. These findings are linked to the literature and discussed through the lens of the themes and evaluative data that emerged from the analysis. 


\section{Developing the Global-Self}

This study was interested in how students participating in a collegiate intramural soccer program perceived the development of their global-self after experiencing interventions intentionally designed to enhance discussion of, and reflection on, attributes of the global-self. The development of the global-self requires the ability to know your self-in-the-world and the ability to act-in-the-world. Three of the themes that emergedhumanizing, stereotyping, and self-awareness - correlate to the development of the selfin-the world. Two other themes that emerged — communication and teamworkdemonstrate the acquisition of attributes that improve students' ability to act-in-theworld. One theme that emerged — community — relates to both aspects of the global-self. This section starts with a discussion of the importance of community, then discusses the data related to self-in-the-world, and closes with a discussion of the data related to act-inthe world.

Community. Communities are both a setting for developing the self-in-the-world and a value that is important to acting-in-the-world. Working in community improves student learning (Allport, 1954; Killick, 2013; Vygotsky, 1958) by both providing peers to help students move through the zone of proximal development (Vygotsky, 1958) and by creating an environment with facilitated intercultural contact (Killick, 2013). The ability to interact in a community is an act-in-the-world attribute that requires students to develop skills that they cannot develop if they are constantly working on their own without the friction or cooperation that comes from working with others.

In the study results, students reported that the development of community was an essential benefit to their participation in intramural soccer. Four communities appear to 
have developed during the study. The main community was the totality of all the student players and staff who engaged in the intramural season. From this community, three smaller communities emerged: the student staff community, the individual team community, and the game community.

The student staff developed as their own community due to their shared experience implementing the actual games and needing to enforce policies. There were two student staff members who identified as being international, and they both created opportunities for the student staff to discuss and reflect on their preconceived ideas about international students. The data suggest that these opportunities created an opportunity to overcome stereotypes.

Another community that emerged was each individual team. While this was not an unexpected outcome, what was unexpected was that students reported that their teams became closer due to the interventions. Team members got to know each other better after answering questions about themselves each week. With each successive week's question, teammates were exposed to more information about each other and developed a deeper understanding of their peers. This unintended outcome, however, provided a space for teams to develop into stronger communities where they learned to rely on each other and to improve their ability to communicate-both important attributes of the global-self.

The design of the study was intended to facilitate interactions so that when two teams introduced themselves to each other and then played against each other, another community—a "game" community—developed. This form of community was thought of as an essential element to providing interactions to improve the development of the 
global-self. Knowing that sports can create difference (Wann \& Grieve, 2005), the formation of a shared community between teams was essential to helping students develop their global-self. The data support that this shared community formed and led to improved humanization and decreased stereotyping.

Communities are also an integral place in which to develop the skills to act-inthe-world. As discussed in Chapter 4, students reported that they learned to "work together," were "able to talk about stuff," and "learn[ed] about different cultures" as a result of their improved communities. These are powerful outcomes that demonstrate the value resulting from improved communities.

Self-in-the-world. The data suggest that the study interventions activated attributes in students that helped them move toward their self-in-the-world by helping them develop self-awareness and by reducing stereotyping and improving humanization. Developing the self-in-the-world, as reviewed in the literature, is a demanding goal. The ability to truly see others as equally human may appear easy, but as we see in the trappings of everyday life, it is clearly not an attribute put into practice easily; thus, the study results are hopeful.

Developing the attribute of self-awareness is central to the concept of self-in-theworld (Killick, 2015). The results of this study reinforce that students made progress in developing this attribute. Students demonstrated multiple ways that they were moving along the scale of self-awareness. During the in-depth interviews, several students mentioned ways in which their self-awareness improved during the season. Ned noted that he had a "different perspective from other culture[s]"; Quang acknowledged that he was not "the sun and the entire galaxy"; while Helen saw "that they're different . . but 
[they are] realistically ... the exact same, almost.” By reflecting on their experience, students made it clear that they had become aware of the assumptions, stereotypes, and behaviors that impacted their ability to treat others as equally human.

Another good indicator of students' developing a sense of self-awareness was the reporting of conscious decisions to maintain composure during the games. Anger, frustration, and threats to identity are all possible outcomes when players are losing or when their performance is not meeting their expecation (Kerr \& Males, 2010). Yet, the data suggest that due to the interventions, students made choices to "go out of their way" to not get mad or "brushed things off a lot easier." These were not attributes that happened spontaneously; instead, they were reported as consciously done in response to the interventions. While there are many contributing factors to the improved sportsmanship rankings, the improved rankings help support the finding that students did a better job de-escalating through their self-awarness than they had in the previous year. Being more self-aware can help reduce stereotyping, a second indicator of improved selfin-the-world.

Stereotyping "runs counter to a project for developing the global student or enacting the global-self' (Killick, 2015, p. 91). Thus, it is essential to the development of the self-in-the-world to create situations in which students learn to identify when and how they stereotype. The interventions appear to have helped create such situations. The data also suggest that most students participating in the intramural soccer season thought they did not stereotype other players, as they consistently reported that they believed they treated others as equally human. Many students pointed to the inherent nature of soccer being a global sport as proof that they did not stereotype. It was as if students thought that 
because they participated in an activity that involved so many international students and that occurred so broadly around the world, that it automatically meant they did not stereotype. This is an idealistic view and perhaps a bit naïve. The data painted a more complex picture, including instances of stereotyping by gender and national origin.

On one hand, most students reported that their stereotyping of others was reduced through their participation in the intramural program, yet there were still cases where students felt stereotyped. For example, one student referee reported being stereotyped by players based on his national origin. Both domestic and international students selfreported stereotyping in their survey responses and interviews, attributing a lack of skill or knowledge to the national origin or gender of a referee.

Both female referees and female players experienced stereotyping. Female players reported feeling stereotyped by the male players in relation to their playing ability or knowledge of the rules. A female referee described being stereotyped by a number of teams, but most often from predominantly Middle Eastern teams. Paradoxically, despite being stereotyped, the females in the league demonstrated high self-awareness in their survey and interview responses, acknowledging in more than one case that they themselves had been guilty of stereotyping international students. Female participants brought multiple identities to this study and the overlap of gender, ethnicity, and nationality created many opportunities to develop the mature interpersonal competence for which Perez et al. (2015) advocate; this is accomplished by "display[ing] integrated social identities and understand[ing] that multiple social identities are part of self and others" (p. 768). If the intent of the global-self is to view others as equally human, reducing the amount of gender stereotyping will need to improve. 
While there were instances of stereotyping, the intramural soccer season appears to have improved the ability of students to reduce their stereotyping of others. Most of the students who participated in the in-depth interviews identified that they had changed their opinion of a culture due to their participation. The study interventions were not designed to remove stereotyping, but rather they were designed to improve the intercultural interactions between players to set the stage for later self-reflection on their beliefs. This goal appears to have been met and the results of the study imply that most students improved the way they viewed others through a reduction in stereotyping, paving the way to truly humanizing the other.

Finally, the interventions appear to have enhanced the opportunity in the inherent nature of soccer to humanize fellow players, an outcome in line with improving the selfin-the-world. Unexpectedly, one of the main findings was that players ended up humanizing the referees much more than they anticipated. This appears to have happened for two reasons. First, the teams interacted with the referees during the pre-game interventions multiple times over multiple weeks, creating more opportunities for breaking down barriers. Second, an internal policy of the intramural program allowed referees to participate as players too, providing another opportunity for interaction. A common contribution to poor sportsmanship in intramurals is that players are often being refereed by their peers. The study results indicate that while there was some pushback to this practice, it created more humanizing of the referees.

While not everybody who participated in the intramural season reported that they had improved their ability to humanize the other, notably there were no reports in the data that participants' views of others had lessened. Since sports can bring out the negative 
side of people, one possible outcome would be dehumanizing, but the data suggest that this did not happen in this study.

Playing a sport can create a sense of sameness, but sometimes it is not enough of a commonality to overcome differences when conflict arises on the field. Due to soccer's role in the world, and the broad range of nationalities and ethnicities that played, there was a danger that the pre-game interventions and post-game socials might have highlighted or created differences instead of similarities. Instead students reported that the interventions helped them develop self-in-the-world by realizing that other players and student staff were, essentially, equally human. This is a substantial finding and an encouraging result.

Act-in-the-world. Successfully acting-in-the-world includes having knowledge, skills, and values that allow a student to "take deliberative action with appropriate references to the contexts and participants implicated in the action" (Killick, 2015, p. 31). Two interdependent themes emerged from the data related to student's ability to act-inthe-word-communication and teamwork. Communication is an important attribute of successful teamwork, yet communication stands as its own theme as it is essential to successful interactions well beyond teamwork. Killick (2015) noted in his definition of the global-self that if a student sees herself as

someone who can engage in respectful dialogue with someone from a different culture [she] is more likely to be able to visualize herself as someone who does engage in respectful dialogue with someone from another culture than the student who does not recognize herself as having that capability. (p. 32)

The two themes built on each other in the data to emerge as important act-in-the-world attributes that students developed during their participation in the intramural season. 
The first act-in-the-world attribute identified was communication. This study helped students improve their communication skills by facilitating communication between players from different teams, among players on the same team, and between players and student staff. Communication between students was initiated on a known shared interest — soccer — and subsequently improved by the uncovering of additional shared interests through the multiple interventions.

The data indicate that improved communication ran through all facets of the study, although it is hard to distinguish communication that improved because of simply playing together from communication that improved due to the interventions. Sam's insight from his in-depth interview provides evidence that the pre-game introductions were important to improving communication when he observed that even a "glimpse into someone else's life" helped there be "better sportsmanship ... [more so] than in any of the past seasons." He expressed that it was "noticeable . . . how teams resolve[d] conflict with each other," suggesting that the prompts provided to students during the pre-game intervention and the simple discussions that occurred during the post-game social had positive effects.

Sam's insights are not too surprising given that in team sports, as suggested by Mishra et al. (2016):

Effective communication is apparent when team members listen to one another and attempt to build on each other's contributions. In team situations, where disagreement is likely to occur, the ability to recognize and resolve conflict is important to the team's success. The creation of feelings of trust, respect, and understanding are key elements in team development. (p. 215)

Communication is a part of good teamwork and the ability to successfully communicate is essential to the ability to act-in-the-world. 
Students improved their teamwork by participating in the intramural soccer league. While teamwork happens in almost all aspects of life, including where we work, where we pray, and where we socialize, teamwork has a strong association with where we play. Successful teamwork relies on several different skills, and students reported learning two: trust and cooperative learning.

Trust is an important aspect of successful teamwork and a value important to developing the ability to act-in-the-world. Killick (2015) describes the importance of trust in developing the ability to act-in-the-world by asserting:

In any group work context, successful participation is founded upon trust. Establishing mutual trust is made more complex for our students when they are among diverse others, and the anxiety and uncertainty stresses associated with cultural immersion ... . are more salient. (p. 163)

The study design helped improve trust between participants. Starting with the student staff training, students interacted with others who were different than them and started to build trust. In prior seasons, there had been conflict between student staff and players from the Middle East. To address that conflict, two Middle Eastern students spoke with the student staff prior to the season, and student staff reported that it decreased their uncertainty about interacting with these different students, thereby increasing trust:

Having the students come in from different countries and share their perspective on soccer was really helpful. I was able to understand how important soccer is to them, and how big of a role it plays in their everyday lives. It made me more understanding of how they act on the field. It also made me understand why they may react to some calls they do.

The observations of pre-game introductions support this survey response as the student staff appeared comfortable and willing to engage with the Middle Eastern students. 
One unanticipated outcome was trust; players on the same team frequently reported that their trust of their teammates improved. The interventions were designed without taking this potential outcome into consideration. In retrospect, it is clear that by engaging with your team multiple times, improved trust should have been an anticipated outcome. Because many of the teams were diverse, this result provides even more reason to consider the study successful. Both the survey responses and the in-depth interviews repeatedly referenced this growth in trust, and pre-game introductions were frequently mentioned as being essential to this improvement.

Improved trust can lead to better cooperative learning, another indicator of positive teamwork. Although, the reliance of sports on individual skill can lead away from cooperative learning, the data indicate that players were excited to learn from each other, particularly as it related to how they played together as teams and how they played against the other team. The data also suggest that players developed more cooperation with the student staff and began to understand that the student staff were doing the best they could in somewhat tense times.

Finally, an unanticipated outcome was that the techniques used resulted in not only intramural players learning more about players from the other team, but also learning more about their own team and the student staff. The interventions helped improve teamwork and communication among already known groups.

\section{Developing the Program}

This second research question of the study asked how students experience an intramural soccer program designed to encourage the development of global-self characteristics. Even though the ultimate outcome was for students to improve their 
concept of global-self, if the study participants had a negative experience, it might suggest that the interventions were not worth repeating. The reporting of the experience of the program differed slightly depending on whether the respondent was a player or a student staff member, but overall the data indicate that students had fun and appreciated the chance to engage with other players and student staff in a way that was different than typical intramural leagues.

Student employees' perceptions of their experience were positive, though not to the level desired. There were multiple student staff who engaged with the opportunities and "leaned in" during the trainings and the interventions, and there were some staff who appeared bored and disinterested during the entire season. During the first staff training session, for example, it was clear that a few student staff were unsure about the value of the interventions, as they were hesitant to talk and appeared annoyed. Similarly, the two student coordinators displayed initial uncertainty about the new requirements, but they remained helpful despite their hesitancy. Since the coordinators were without a supervisor, they communicated concerns about the details of implementing the intramural season and were at first more concerned with the player experience than with learning and supporting the introduction of new game management techniques. However, by working closely with the student coordinators to make modifications to the captain's meeting, refining the questions asked at the pre-game social, and modifying the timing of the post-game socials, the student coordinators got on board with the proposed changes and ultimately were helpful. Working closely with the student staff and incorporating their feedback was essential to the success of the interventions. 
In the in-depth interviews with student staff, there was a high level of acceptance and excitement about the interventions. In the survey results, however, employees were less enthusiastic and shared that facilitating the interventions was a chore and not appreciated by the players. These student staff perceptions about players' reactions to the interventions are important to acknowledge. A couple of student staff reported that they thought the interventions were a "waste of time" and that players "just wanted to play." Some players also reported the activities were "generally awkward" and they "would have rather started right away." However, a clear majority of players disagreed with these sentiments and reported that they found benefit in the interventions, leading to the conclusion that there was a high return on the time invested. The perceptions of student staff might have improved if they had been better informed on the positive outcomes of the interventions and the rationale for the activities, and if schedules had been created to account for the time required to do the activities. In contrast to the student staff, players appeared to be more curious. The interventions were generally accepted as beneficial to the player experience, although some interventions were more successful than others. Reviewing each intervention will provide context for the player's experience.

The captains' meeting about the global-self and the modifications in game management saw multiple players lean in and engage with the information. There was a shift in the energy from what had been tepid player engagement with the training. These results are similar to the observations of the pre-game introductions. During the pre-game introductions, the referees were successful in getting the players to congregate at the middle of the field and to line up the two teams in a circle to do the introductions and answer the pre-game question. Despite a very wet and cold season, players engaged with 
the pre-game questions. While players displayed different levels of engagement, it was clear in almost every pre-game observed that players took the time to listen and to share something of substance. There were no instances where players refused to participate or vocally objected to participating. The data analysis confirmed these observations and demonstrated that the interventions were positively accepted. Ultimately, this is a very important finding as the pre-game introduction proved to be the most impactful of the interventions.

In contrast to the success of the pre-game introductions, the post-game socials were a mixed result. It is important to note the significant differences between the two post-game socials as discussed in the methodology. Due to the use of community action research, the second post-game intervention was much more successful than the first. Changing how players were informed about the post-game social and how far they had to walk to participate had a big difference on their engagement. When players had to walk 35 yards to participate and receive a piece of pizza they were much less likely to participate. Instead, they engaged to a much higher degree when their reward for participating was candy that was given to them right on the field. On the whole, for those players that participated in the post-game socials, they reported greater connections with other players and improved intercultural understanding.

Just as Maslow (1942) argued that safety is one of the building blocks of selfactualization, keeping players and employees safe is a basic need of intramural programs. Without a safe environment, students would not participate, enjoy, or learn anything through their participation in intramurals. Sportsmanship scores can be used to assess the safety of an intramural program. In addition to improved safety, good sportsmanship 
scores for intramural sports can indicate meaningful outcomes that include clarifying values (Rothwell \& Theodore, 2006), improving risk management (Stier, Schneider, Kamp, Haines, \& Gaskins, 2008), and reducing conflict (Vincent \& Kearney, 2001). These outcomes, in turn, support stronger communities and happier players and student staff. The improved sportsmanship scores resulting from the interventions imply that the environment supported improved outcomes related to the global-self.

In addition to the positive impacts on student development, the results of the study indicate that the interventions ended up creating a better work environment for the intramural student staff. Sports inherently create winners and losers, and this often leads to a difficult environment in which to work. Refereeing sports requires the ability to handle conflict, work with peers, and hold others accountable. By creating an environment where there is better sportsmanship, intramural student staff and players will have less stress, thus creating improved experiences that are unavailable if they are involved in conflict.

\section{Implications for Practice}

The results of the study apply to the practices of professionals in collegiate recreation and to the day-to-day management techniques of intramural sport programs. The following section will discuss the implications for intramural sports programs, provide recommendations to improve the interventions, and will conclude with a review of the contribution of the study to improving the competencies of collegiate recreation professionals.

Looking beyond intramural sports, the basic tenets of the study can apply to programs and topics supporting student development in campus recreation and other 
student affairs areas. For instance, similar practices could be applied to outdoor program trips or club sports activities to support the development of the global-self among participants. In the example of outdoor programs, almost all aspects of this study can be applied to the design and implementation of adventure trips. Adventure trips involve training of student staff, the development of teamwork for trip participants, the opportunity to have discussions beyond basic introductions, and the chance for program assessment. Clubs may not be able to do all steps of the intervention, but it is possible that a program's club teams could implement pre- and post-game activities comparable to the interventions used in this study. More self-directed activities, like group exercise classes or swimming, may be a more difficult environment in which to implement these strategies, but the opportunity to use some of these techniques exists in pre-game introductions, the training of student staff, and intentional efforts to create communities.

A carefully designed collegiate recreation program can make a difference in the development of students' global-self, but relying simply on any one activity to create perceived change is not enough. Instead, a wrap-around approach, including staff training, program modification, and self-reflection, appears to be an effective way to implement perceived change in students. This wrap-around approach could also be modified to apply to other student affairs areas such as student activities, residence life, or international student services.

\section{Recommendations for Improving Interventions}

With four different interventions used in this study, there are many opportunities to improve the manner in which the interventions were designed and implemented. This section reviews potential changes to the interventions. 
Since this was the first season of the intervention, and it was completed in only one sport, it is not surprising that it felt inadequate for some students. More notable change may occur if the interventions are done for longer than five weeks, especially given that for constructivist learning to take place, adequate time for learning is crucial (Hein, 1991). For the learning to be substantive for the students, replicating the interventions over multiple seasons and multiple sports would be helpful and should be considered.

The cold and rainy weather on multiple nights of the interventions seemed to discourage students from participating in the post-game social. Having alternative plans in place in case of bad weather would be important for practitioners to develop in order to have a more successful implementation of the post-game social. As a cost-saving measure, the use of candy instead of pizza seemed to be just as motivating to encourage student participation in the post-game social. In general, the post-game social may yield better results if the location of the intervention is closer to the field or the gym so students can more easily take time to interact with teams other than their own.

The research protocol for the pre-game introduction had both teams come to the middle of the field and engage in the pre-game script and introductions. One variable not explored was the requirement that teams line up interspersed with one another, providing an immediate visual and emotional change to the introductions. The use of scripts was an important aspect to the consistent messaging envisioned for the interventions.

Unfortunately, weather and lack of preparation created several instances where student staff were scrambling to locate the script or recreate it. The resolution to this issue was laminating the pre-game script so that it was easier to keep and use. Consistently 
applying the use of the script is important, as repetition of messages improves learning (Kenner \& Wienerman, 2011).

The need for quality interactions among diverse students is a known factor for increased reporting of intercultural competence (Allport, 1954; Saenz et al., 2007). While the research protocols helped to improve the quality of the interactions between students, there is room to do more. Future research could create more meaningful dialogue by intentionally designing post-season opportunities for students to gather and talk about their experiences. Faculty members with expertise could guide these talks, or they could simply facilitate social activities with guided questions. The better the quality of the experience, the deeper the change (Saenz et al., 2007); thus, creating learning environments where students are invited to a deeper conversation is encouraged.

\section{Implications for the Professional Competency Areas}

The study impacted numerous skills and development areas identified in the NIRSA Core Competencies, particularly as they improve practitioners' knowledge and awareness of the global-self. The implications for competency development are important to the continued improvement of the profession of collegiate recreation. While the current political environment may have chilled the perceived need to develop the skills, knowledge, and values to develop the global-self, the history of higher education and the overall demographic trends strongly suggest that the current climate is a xenophobic blip. This is a time when the values of diversity and inclusion need to be explored and celebrated. These results and the methods used in this study can help collegiate recreation professionals develop their competencies in developing a global perspective, an important NIRSA value. 
NIRSA defines their professional competencies in three categories: programming, philosophy and theory, and personal and professional qualities. Each category is further broken into subsections (see Appendix A). This study did not address all the subsections in each category. There are some subsections of the competencies that relate more strongly than others to the outcomes of this study, and those are grouped together based on their topic, not on their main category. For example, while equity, diversity, and inclusion is a subsection of each competency category, it is discussed in aggregate later in this chapter.

Student learning and development. Practitioners who implement the ideas of this study need to develop or demonstrate the basic theories of student learning and development, including the values of experiential learning (Allport, 1954), reflection (Dewey, 1938), and the global-self (Killick, 2015). By working with students directly in the creation of their intramural programs, by being intentional in their training to broaden topics beyond the necessities of a program, by encouraging students to interact beyond the game, and by talking about global issues, practitioners will demonstrate competence in their knowledge of student learning and development and have opportunities to put them into practice.

This study can also help practitioners implement concrete steps to use student development theory. Among other theories, practitioners will be able to apply Kuh's (1995) student engagement theory that out-of-the-classroom experiences help students gain interpersonal competence through peer interactions. Practitioners can also support students moving through Chickering and Reisser's (1993) developmental vectors and implement theories on intercultural development (Killick, 2015; Perez et al., 2015). 
Hopefully, practitioners will modify the methods used in this study to develop and promote new methods to add to the overall body of work on student development theory.

Teaching and training. Encouraging professionals in collegiate recreation to modify their teaching curriculum and training methods is at the core of this study. By integrating constructivist learning theories, and by having the opportunity to learn about the global-self at-home, practitioners can expand their teaching and training methods beyond the typical intramurals student staff curriculum focused on refereeing techniques and event management.

Program development. At the core of this competency is the goal that practitioners use "intentional planning methods that include development of student learning outcomes" (NIRSA, 2017, p. 4). This study specifically requires that practitioners be thoughtful in the design of their programs to intentionally inspire and create outcomes that are beyond wins and losses or mere participation. Professionals in many collegiate recreation environments can duplicate the programming techniques and assessment methods to improve their competence.

Leadership. This competency area differentiates itself from leadership models based on stereotypical roles associated with governance or team sports. A NIRSA professional demonstrates advanced leadership competency by creating environments where there is support for the "free and open exchange or ideas and beliefs and where issues of power and privilege are identified and addressed" (NIRSA, 2017, p. 4). Instead of focusing on the role of a captain, for instance, this study created spaces where students could practice their own leadership in a multitude of different places. Employees practice supervising other employees, employees provide leadership to players, captains provide 
leadership to teammates, and participants practice leadership in the pre-game introductions or post-game interventions. By facilitating conversations on the global-self, practitioners can move toward creating leadership environments that support this more advanced leadership concept.

Equity, diversity, and inclusion. This competency area is multi-layered and evolving rapidly. Most current research on concepts of equity, diversity, and inclusion focuses on ways to addresses domestic issues related to race, culture, and gender identity. While ethnicity or national origin is often included in the description of areas to address, international students are often overlooked in trainings and conversations. The NIRSA competencies have separate equity, diversity, and inclusion and global perspective competencies. The premise of this study puts into question why there would be separate approaches to working with different students if indeed equity, diversity, and inclusion are desired outcomes. As a practical approach to these competencies, this study should help practitioners "design culturally relevant and inclusive programs, services, policies, and practices," while integrating "cultural knowledge with specific and relevant cultural issues on campus" (NIRSA, 2017, p. 5). This study encouraged integration of this cultural knowledge into collegiate recreation programs. The advanced level of this competency requires that equity, diversity, and inclusion be integrated into an entire recreation department. Implementing the concepts from this study can help set the stage for that to successfully happen.

The basic theories of equity, diversity and inclusion are advocated through this professional competency, and practitioners are encouraged to "teach staff and student employees about issues of equity and diversity and demonstrate their significance in 
collegiate recreation" (NIRSA, 2017, p. 7). This study argues that there is a need to broaden the definition of equity and diversity to include international students and global perspectives, and the results of this study can help practitioners improve the way they understand the importance of basic equity, diversity, and inclusion philosophies.

As leaders in collegiate recreation, this competency requires practitioners to actively pursue opportunities that help them to gain the personal and professional skills required to promote the values of equity, diversity, and inclusion. These skills include the need to work effectively with those from diverse backgrounds, use language that is appropriate for the context, engage in professional development on equity, diversity, and inclusion, and join in environments designed to support equity, diversity, and inclusion. In the end, this study encouraged creating a more level playing field for all participants while encouraging reflection on the role of "culture" in our universities and communities.

Global perspective. The development of a global perspective has been discussed at length in this study. Practitioners who engage with this study and put into practice the interventions will undoubtedly improve their competency in this area. This study encouraged professionals to look closely at how they themselves move through the world and how their work impacts students' self-reflection. From developing their awareness of international differences, to facilitating interactions between people of different international perspectives, to insuring that policies and practices are inclusive of a multicultural community, the implications of this study help professionals move through these attributes. Understanding connection, creating a sense of social and personal responsibility, and then applying these responsibilities are reinforced by the techniques demonstrated in this study. 
Developing their own global perspective is an important attribute for a practitioner to develop if they are going to be a competent professional. This study can help practitioners "develop awareness of international differences" and "distinguish cultural differences and incorporate concepts into programming and/or facility design and operations to educate about the differences" (NIRSA, 2017, p. 10). Practitioners should develop these qualities by implementing the ideas in the study.

While the professional and personal competency areas provide a rationale for helping professionals to further the development of their global-self, the results of this study indicate that a modification or addition to the professional competencies that further defines how we interact with each other in a more meaningful way would be beneficial to the profession.

Trends in recreational sports. The implications of this study have current relevance related to this competency area for two specific reasons. First, in the winter of 2017, NIRSA identified Canada as its first stand-alone international region. NIRSA had been working on this for five years, and professionals now have a concrete reason to think globally. This supports the second emerging trend in the profession, which is the development of a global perspective, as identified in NIRSA's strategic values (NIRSA, 2015)

Student affairs issues and theories. In this competency area, practitioners are urged to use the issues and theories of student affairs in a direct and intentional way in collegiate recreation. As the profession of collegiate recreation has developed, the practice of grounding work in theory is becoming more common. By using this study to 
modify the way trainings and programs are implemented, practitioners can be more mindful of the praxis of their work.

Adaptability. Practitioners should be integrating input from others and being aware that there are ever changing cultural norms when implementing the ideas in this study. The rapidly changing global environment requires professionals to anticipate these changes to cultural norms and proactively address them in ways that include input from others. For example, due to the heightened awareness of immigration, professionals should be aware that they need to have knowledge about immigration issues and identify opportunities to help others learn about the complexities around that issue. Being conscious of, and responsive to, social issues does not normally fall in the purview of collegiate recreation programmers, but this study encourages practitioners to make this part of their work.

\section{Limitations}

Several limitations of this study relate to the transferability of the study results to the broader collegiate recreation profession and should be considered when planning future research on this topic. The first limitation was the inconsistent application of the interventions. Without a full-time person to attend every single one of the study events, there was a lack of consistency in the execution of the pre-game activities; not all participants had the exact same experience. This limitation was offset because the researcher had direct access to the student staff, which allowed for consistent explanations of expectations and one point of contact for questions and communication.

The location for the study at an urban institution with limited playing fields and modest program participation numbers makes the task of scaling the project up hard to 
predict. Most collegiate recreation intramural programs are larger in scale than the one studied, consisting of multiple soccer fields with multiple teams playing on multiple days. The size of the intramural staff, and their limited engagement in the online survey and the in-depth interviews, made it difficult to draw conclusive themes around their shared experience. Similarly, the demographics of the student body at the location of the study also differ from a "traditional" university campus. However, with rapidly expanding access to higher education, the growing importance of community college, and the shifting national student enrollment demographics, this study still holds much to consider for many of today's higher education institutions.

Finally, the backyard nature of this study possibly impacted the analyses and conclusions. While the research techniques used lessened the power relationships, the fact remains that a power relationship existed between the researcher and the study participants. Because this was community-based research, the personality of the researcher may have influenced the participants' perceptions of the study.

\section{Future Research}

Future research on the development of the global-self through collegiate recreation could help identify the transferability of the methods used. Due to the constructivist nature of the study design, future research would most likely be impacted by the skills, personalities, and passions of the researcher. Similarly, by relying so much on community action research methods, any future research will inevitably be influenced by the community in which the research takes place. The following research possibilities are suggested as potential areas based on the current findings. 
While the current study investigated the impact of the interventions on all participants and student staff, with a larger intramural program, it would be possible to narrow the focus to a more narrowly defined category of participants. At a larger institution it would be possible to focus on the impact of the interventions on the perceptions of the referees, or the perceptions of identified international students, or even on different perceptions based on gender identity. By narrowly identifying the study group, future research may help practitioners to refine how they communicate and the tools they use to create the global-self.

Another area of further study could be the relationship between interventions designed to impact the global-self and the impact they have on sportsmanship and perceived sportsmanship. In the field of intramurals, sportsmanship has often been the focus of research (Haines \& Fortman, 2008; Mathner, Martin, Tatum, \& Chouti, 2010; Rothwell \& Theodore, 2006), and these types of interventions could be duplicated or modified to study their potential impact on this important aspect of student development.

Finally, there is a wide range of learning environments outside of collegiate recreation that could benefit from similarly designed interventions. Communities ranging from student organizations to residence halls to academic classrooms, could implement these interventions in staff training, peer interactions, and in-depth reflections and they could be investigated for their possible impact on the development of the global-self. It would also be possible to replicate the interventions in areas outside of universities, particularly in youth or community recreation in order to assess if similar changes in global-self occur. 


\section{Conclusion}

Multiple intersecting factors led to the undertaking of this study. NIRSA's global perspective value was being developed when the idea for the study was initiated. The study was designed in response to what was, at least until the Presidential Election of 2016, the rapidly increasing internationalization and globalization of higher education. Sports, particularly intramural soccer, was the research setting due to both its global importance and its inherent impact on intercultural interactions. While the impact that soccer has on the world is complex and can be weighed down with negative connotations, the opportunity to use the vehicle of sport for good helped form the purpose of this study.

The learning demonstrated in the findings of this study, and the vocal and visible support from student employees, indicate that the interventions were successful in improving student behavior, modifying student perceptions of their global-self, and impacting the learning associated with participation. Did this study result in removing stereotypes or eliminating bad behavior? No, it did not. Yet, this study did improve sportsmanship, it increased the self-reflection of participants, it showed that participants recognized elements of the global-self in themselves, and it stimulated a shift toward greater acceptance between students from diverse backgrounds.

The engagement with the participants went even better than expected. Starting with the captains' meeting, the level of engagement in the intramural soccer program was higher than it had been for several years. Creating a more meaningful reason for students to engage with each other appears to be a successful technique for creating positive outcomes. 
This study successfully modified participant behavior through a wrap-around approach that included staff training, participant training, pre- and post-game interventions, and the use of reflection. While this study took place at an urban institution, the methods used are scalable and can be replicated at universities of any size. Students reported that the design of the study resulted in an improved experience of intramurals. Students communicated that they enjoyed the pre- and post-game interventions. They described an improvement in how they interacted with other students, and they overwhelmingly expressed a positive impression of the intramural program as it was implemented. With these positive results in mind, it is no surprise that sportsmanship scores increased across all aspects of the intramural soccer program.

Finally, multiple themes emerged from the analysis that highlighted students' movement toward a more global-self. Students' demonstrated an improved self-in-theworld by reporting increased self-awareness, improved recognition of stereotyping, and the acknowledgment of humanizing of their fellow students. Students increased their ability to act-in-the-world through their improved communication and demonstrating attributes of strong teamwork. These global-self qualities were essential to helping students create, and participate in, healthier communities. These themes, combined with the evaluative data, demonstrate that students perceived an improvement in their globalself through their participation in a collegiate recreation intramural soccer program. 
References

Allen, J. T., Drane, D. D., Byon, K. K., \& Mohn, R. S. (2010). Sport as a vehicle for socialization and maintenance of cultural identity: International students attending American universities. Sport Management Review, 13, 421-434. doi:10.1016/j.smr.2010.01.004

Allport, G. (1954). The nature of prejudice. Cambridge, MA: Perseus Books.

Altbach, P. G., \& Knight, J. (2007). The internationalization of higher education: motivations and realities. Journal of Studies in International Education, 11, 290305. doi:10.1177/1028315307303542

American College Personnel Association (ACPA) \& National Association of Student Personnel Administrators (NASPA). (2015). Professional competency areas for student affairs educators. Washington, DC: Authors. Retrieved from https://www.naspa.org/images/uploads/main/ACPA_NASPA_Professional_Comp etencies_FINAL.pdf

Applefield, J. M., Huber, R., \& Moallem, M. (2001). Constructivism in theory and practice: Toward a better understanding. High School Journal, 84(2), 35-53. Retrieved from http://www.jstor.org/stable/pdf/40364404.pdf

Artinger, L., Forrester, S., Hunt, C., Meigs, M., Milord, N., \& Sampson, B. (2006). The social benefits of intramural sports. NASPA Journal, 43(1), 69-87.

Astin, A. (1975). Preventing students from dropping out. San Francisco, CA: Jossey-Bass.

Astin, A. (1984). Student involvement: A developmental theory for higher education student involvement. Journal of College Student Development, 25, 297-308. 
Astin, A. (1999). Student involvement: A developmental theory for higher education student involvement. Journal of College Student Development, 40, 518-529.

Athanasios, L. (2005). Communication problems in professional sports: the case of Greece. Corporate Communications: An International Journal, 10, 252-256. doi:10.1108/13563280510614500

Austin, K., Orcutt, S., Rosso, J., \& Darling-Hammond, L. (2001). How people learn: Introduction to learning theories. The Learning Classroom: Theory Into Practice - A Telecourse for Teacher Education and Professional Development. Stanford, CA: Stanford University, School of Education. Retrieved from http://www.seas.upenn.edu/ eas285/Readings/Hammond_HowPeopleLearn.pdf

Baxter Magolda, M. B. (2003). Identity and learning: Student affairs' role in transforming higher education. Journal of College Student Development, 44, 231-247. doi:10.1353/csd.2003.0020

Beamon, K. (2014). Racism and stereotyping on campus: Experiences of African American male student-athletes. Journal of Negro Education, 83, 121-134. doi:10.7709/jnegroeducation.83.2.0121

Bermen, R. (2015). Donald Trump's call to ban Muslim immigrants. The Atlantic. Retrieved from http:/www.theatlantic.com/politics/archive/2015/12/donaldtrumps-call-to-ban-muslim-immigrants/419298/

Braskamp, L. A. (2008). Developing global citizens. Journal of College and Character, 10(1), 1-5. doi:10.2202/1940-1639.1058 
Braskamp, L. A., Braskamp, D. C., Merrill, K. C., \& Engberg, M. E. (2008). Global Perspective Inventory (GPI): It's purpose, construction, potential uses, and psychometric characteristics. Chicago, IL: Global Perspective Institute.

Bruner, R. F., \& Iannarelli, J. (2011). Globalization of management education. Journal of Teaching in International Business, 22, 232-242.

doi:10.1080/08975930.2011.653908

Burkard, A., Cole, D. C., Ott, M., \& Stoflt, T. (2005). Entry-level competencies of new student affairs professionals: A Delphi study. NASPA Journal, 42, 283-309. doi:10.2202/1949-6605.1509

Burney, S. (2012). Pedagogy of the other: Edward Said, postcolonial theory, and strategies for critique. New York, NY: Peter Lang.

Busby, T. L. A. (2011). An exploration of campus recreation's role in student engagement (Master's thesis). Retrieved from ProQuest Dissertations and Theses Global database. (Order No. MR93453)

Caruana, V., \& Ploner, J. (2010a). Internationalisation and equality and diversity in higher education: Merging identities. Leeds, England: Leeds Metropolitan University, Equality Challenge Unit.

Chang, J. (2006). A transcultural wisdom bank in the classroom: making cultural diversity a key resource in teaching and learning. Journal of Studies in International Education, 10, 369-377. doi:10.1177/1028315306287905 
Charles-Toussaint, G. C., \& Crowson, H. M. (2010). Prejudice against international students: The role of threat perceptions and authoritarian dispositions in U.S. students. Journal of Psychology, 144, 413-428. doi:10.1080/00223980.2010.496643

Chickering, A., \& Braskamp, L. A. (2009). Developing a global perspective for personal and social responsibility. Peer Review, 11(4), 27-31.

Chickering, A., \& Reisser, L. (1993). Education and identity (2 ${ }^{\text {nd }}$ ed.). San Francisco, CA: Jossey-Bass.

Childress, L. K. (2009). Internationalization plans for higher education institutions. Journal of Studies in International Education, 13, 289-309. doi:10.1177/1028315308329804

Cho, J., \& Yu, H. (2014). Roles of university support for international students in the United States: Analysis of a systematic model of university identification, university support, and psychological well-being. Journal of Studies in International Education, 19(1), 11-27. doi:10.1177/1028315314533606

Colby, A., \& Sullivan, W. M. (2009). Strengthening the foundations of students' excellence, integrity, and social contribution. Liberal Education, 95(1), 22-29. Retrieved from http://files.eric.ed.gov/fulltext/EJ861148.pdf

Coryell, J. E., Durodoye, B. A., Wright, R. R., Pate, P. E., \& Nguyen, S. (2012). Case studies of internationalization in adult and higher education: Inside the processes of four universities in the United States and the United Kingdom. Journal of Studies in International Education, 16, 75-98. doi:10.1177/1028315310388945 
Creswell, J. W., Hanson, W. E., Plano Clark, V. L., \& Morales, A. (2007). Qualitative research designs: Selection and implementation. Counseling Psychologist, 35, 236-264. doi:10.1177/0011000006287390

Croom, P. W. (2014). Internationalization and institutional strategy. AUDEM: The International Journal of Higher Education and Democracy, 3, 99-119. doi:10.1353/aud.2012.0007

Cross, C. (2014). Political education: Setting the course for state and federal policy (2 $2^{\text {nd }}$ ed.). New York, NY: Teachers College Press.

Crowther, P., Joris, M., Otten, M., Nilsson, B., Teekens, A., \& Wachter, B. (2000). Internationalisation at home: A position paper. Amsterdam, Netherlands: Drukkerij Redbaiter.

Dalton, J. C. (1999). The significance of international issues and responsibilities in the contemporary work of student affairs. New Directions for Student Services, 1999(86), 3-11. doi:10.1002/ss.8601

De Vita, G. (2000). Inclusive approaches to effective communication and active participation in the multicultural classroom. Active Learning in Higher Educationin Higher Education, 1, 168-180. doi:10.1177/1469787400001002006

De Wit, H. (2002). Internationalization of higher education in the United States of America and Europe: A historical, comparative, and conceptual analysis. Westport, CT: Greenwood Press.

Deardorff, D. K. (2006). Identification and assessment of intercultural competence as a student outcome of internationalization. Journal of Studies in International 
Education, 10, 241-266. doi:10.1177/1028315306287002

Dewey, J. (1933). How we think: A restatement of the relation of reflective thinking to the educative process. Boston, MA: Heath and Company.

Donahue, E. G., Rip, B., \& Vallerand, R. J. (2009). When winning is everything: On passion, identity, and aggression in sport. Psychology of Sport and Exercise, 10, 526-534. doi:10.1016/j.psychsport.2009.02.002

Doty, J. (2006). Sports build character?! Journal of College and Character, 7(3), 1-9, doi: $10.2202 / 1940-1639.1529$

Durkheim, E. (1961). Suicide. Glencoe, IL: The Free Press.

Duţă, N. (2015). From theory to practice: The barriers to efficient communication in teacher-student relationship. Procedia - Social and Behavioral Sciences, 187, 625-630. doi:10.1016/j.sbspro.2015.03.116

Eaton, P. W. (2016). The competency-based movement in student affairs: Implications for curriculum and professional development. Journal of College Student Development, 57, 573-598. doi:1353/csd.2016.0061

Enders, J. (2004). Higher education, internationalisation, and the nation-state: Recent developments and challenges to governance theory. Higher Education, 47, 361382. doi:10.1023/B:HIGH.0000016461.98676.30

Featherstone, M. (1990). Global culture: Nationalism, globalization, and modernity. London, England: Sage.

Feltz, D. L., Schneider, R., Hwang, S., \& Skogsberg, N. J. (2013). Predictors of collegiate student-athletes' susceptibility to stereotype threat. Journal of College Student Development, 54, 184-201. doi:10.1353/csd.2013.0014 
Forrester, S. (2015). Benefits of collegiate recreational sports participation: Results from the 2013 NASPA assessment and knowledge consortium study. Recreational Sports Journal, 39(1), 2-15. doi:10.1123/rsj.2015-0005.

Gacel-Ávila, J. (2005). The internationalisation of higher education: A paradigm for global citizenry. Journal of Studies in International Education, 9, 121-136. doi:10.1177/1028315304263795

Garraway, J., \& Volbrecht, T. (2011). Theorising experiential learning. South African Journal of Higher Education, 25, 1,091-1,102. Retrieved from http://hdl.handle.net/10520/EJC37736

Giulianotti, R. (2011). Sport, peacemaking and conflict resolution: A contextual analysis and modelling of the sport, development and peace sector. Ethnic and Racial Studies, 34, 207-228. doi:10.1080/01419870.2010.522245

Glass, C., Buss, S., \& Braskamp, L. A. (2013). Uneven experiences: What's missing and what matters for today's international students. Chicago, IL: Global Perspectives Institute.

Glass, C. R., Gómez, E., \& Urzua, A. (2014). Recreation, intercultural friendship, and international students' adaptation to college by region of origin. International Journal of Intercultural Relations, 42, 104-117. doi:10.1016/j.ijintrel.2014.05.007

Glesne, C. (2016). Becoming qualitative researchers: An introduction (5 $5^{\text {th }} \mathrm{ed}$.). New York, NY: Pearson.

Grover, K. (2014). Experiential learning and its role in training and improved practice in high level sports officiating. Journal of Research in Education, 4, 90-102. Retrieved 
from http://files.eric.ed.gov/fulltext/EJ1098191.pdf

Gundlach, M., Zivnuska, S., \& Stoner, J. (2006). Understanding the relationship between individualism-collectivism and team performance through an integration of social identity theory and the social relations model. Human Relations, 59, 1,603-1,632. doi:10.1177/0018726706073193

Guo, Q., \& Ross, C. M. (2014). An exploratory study of asian international students' campus recreational sports participation. Recreational Sports Journal, 38(1), 5568. doi:10.1123/rsj.2013-0007

Gurin, P., Dey, E. L., Hurtado, S., \& Gurin, G. (2002). Diversity and higher education: Theory and impact on educational outcomes. Harvard Educational Review, 72, 330-366. doi:10.1353/rhe.0.0172

Haines, D. J., \& Fortman, T. (2008). The college recreational sports learning environment. Recreational Sports Journal, 32(1), 52-61. doi:10.1123/rsj.32.1.52

Haines, D. J., \& Fortman, T. (2009). Validation of the collegiate recreational sports core competenices using delphi methodology. Columbus, OH: National Research Institute for College Recreational Sports and Wellness.

Haslam, N., \& Bain, P. (2007). Humanizing the self: Moderators of the attribution of lesser humanness to others. Personality \& Social Psychology Bulletin, 33, 57-68. doi:10.1177/0146167206293191

Hazen, H. D., \& Alberts, H. C. (2006). Visitors or immigrants? International students in the United States. Population, Space and Place, 12, 201-216. doi:10.1002/psp 
Hein, G. E. (1991). Constructivist learning theory: The museum and the needs of people. Presented at the International Committee of Museum Educators (CECA) Conference, Jerusalem, Israel. Retrieved from http://www.exploratorium.edu/ifi/resources/constructivistlearning

Hellsten, M., \& Prescott, A. (2004). Learning at university: The international student experience. International Education Journal, 5, 344-351.

Henderson, K. A. (2014). The imperative of leisure justice research. Leisure Sciences, 36, 340-348. doi:10.1080/01490400.2014.916971

Hendrickson, R., Lane, J., Harris, J., \& Dorman, R. (2013). Acadmic leadership and governance in higher education. Sterling, VA: Stylus.

Herdlein, R. (2004). Survey of chief student affairs officers regarding relevance of graduate preparation of new professionals. NASPA Journal, 42(1), 51-71.

Herdlein, R., Riefler, L., \& Mrowka, K. (2013). An integrative literature review of student affairs competencies: A meta-analysis. Journal of Student Affairs Research and Practice, 50, 250-269. doi:10.1515/jsarp-2013-0019

Himanka, J. (2012). The university as a community of selves: Johan Vilhelm Snellman's On Academic Studies. Higher Education, 64, 517-528 doi:10.1007/s10734-0129508-5

Heo, J., \& Lee, Y. (2007). “I don't want to feel like a stranger”: Korean students who play basketball seriously. Leisure, 31, 133-154.

doi:10.1080/14927713.2007.9651376 
Ho, E., Bulman-Fleming, B., \& Mitchell, B. (2003). Course internationalization:

Engaging students as learning resources (Unpublished manuscript). Presented at the annual conference of the Canadian Bureau for International Education, University of Waterloo, Ontario, Canada.

Hogg, M., Terry, D., \& White, K. (1995). A tale of two theories: A critical comparison of identity theory with social identity theory. Social Psychology Quarterly, 58, 255-269. doi: $10.2307 / 2787127$

Höglund, K., \& Sundberg, R. (2008). Reconciliation through sports? The case of South Africa. Third World Quarterly, 29, 805-818. doi:10.1080/01436590802052920

Hser, M. P. (2005). Campus internationalization: A study of American universities' internationalization efforts. International Education, 35(1), 35-48.

Institute on International Education. (2014). Open Doors Report on International Educational Exchange. Retrieved from https://www.iie.org/Research-andInsights/Open-Doors

Johnson, C. W. (2014). "All you need is love": Considerations for a social justice inquiry in leisure studies. Leisure Sciences, 36, 388-399. doi:10.1080/01490400.2014.917006

Johnson, J. P., Lenartowicz, T., \& Apud, S. (2006). Cross-cultural competence in international business: Toward a definition and a model. Journal of International Business Studies, 37, 525-543. doi:10.1057/palgrave.jibs.8400205

Johnson, M. (2014). Predictors of college students engaging in social change behaviors. Journal of College and Character, 15(3), 37-41. doi:10.1515/jcc-2014-0019 
Jon, J.-E. (2013). Realizing internationalization at home in Korean higher education: Promoting domestic students' interaction with international students and intercultural competence. Journal of Studies in International Education 17, 455470. doi:10.1177/1028315312468329

Jones, E., \& Killick, D. (2013). Graduate attributes and the internationalized curriculum: embedding a global outlook in disciplinary learning outcomes. Journal of Studies in International Education, 17, 165-182. doi:10.1177/1028315312473655

Kaiser, K. (2009). Protecting responsdent confidentiality in qualitative research. Qualitative Health Research, 19, 1,632-1,641. doi:10.1177/1049732309350879

Karkouti, I. M. (2015). The role of student affairs practitioners in improving campus racial climate: A case study. College Student Journal, 29, 31-39.

Kegan, R. (1994). In over our heads: The mental demands of modern life. Cambridge, MA: Harvard University Press.

Keim, M. (2003). Nation building at play: Sport as a tool for social integration in postapartheid South Africa. Aachen, Germany: Meyer \& Meyer.

Kenner, C., \& Weinerman, J. (2011). Adult learning theory: Applications to nontraditional college students. Journal of College Reading and Learning, 41, 87-96.

Kerr, J. H., \& Males, J. R. (2010). The experience of losing: Qualitative study of elite lacrosse athletes and team performance at a world championship. Psychology of Sport and Exercise, 11, 394-401. doi:10.1016/j.psychsport.2010.04.014

Kerr, J. H., Wilson, G. V., Nakamura, I., \& Sudo, Y. (2005). Emotional dynamics of soccer fans at winning and losing games. Personality and Individual Differences, 
38, 1,855-1,866. doi:10.1016/j.paid.2004.10.002

Kidd, B. (2008). A new social movement: Sport for development and peace. Sport in Society, 11, 370-380. doi:10.1080/17430430802019268

Killick, D. (2012). Seeing ourselves-in-the-world: Developing global citizenship through international mobility and campus community. Journal of Studies in International Education, 16, 372-389. doi:10.1177/1028315311431893

Killick, D. (2013). Global citizenship, sojourning students and campus communities. Teaching in Higher Education, 18, 721-735. doi:10.1080/13562517.2013.836087

Killick, D. (2015). Developing the global student: higher education in an era of globalization. New York, NY: Routledge.

Kim, J. (2012). Exploring the experience of intergroup contact and the value of recreation activities in facilitating positive intergroup interactions of immigrants. Leisure Sciences, 34, 72-87. doi:10.1080/01490400.2012.633856

King, P. M., \& Baxter Magolda, M. B. (2005). A developmental model of intercultural maturity. Journal of College Student Development, 46, 571-592. doi:10.1353/csd.2005.0060

Knight, J. (2004). Internationalization remodeled: definition, approaches, and rationales. Journal of Studies in International Education, 8, 5-31. doi:10.1177/1028315303260832

Knight, J., \& De Wit, H. (1997). Internationalisation of higher education: A conceptual framework. In J. Knight \& H. De Wit (Eds.), Internationalisation of higher education in Asian Pacific countries (pp. 5-18). Amsterdam, Netherlands: EAIE/OECD. 
Kuh, G. D. (1993). In their own words: What students learn outside the classroom. American Educational Research Journal, 30, 277-304. Retrieved from http://www.jstor.org/stable/1163236

Kuh, G. D. (1995). The other curriculum: Out-of-class experiences associated with student learning and personal development. Journal of Higher Education, 66, 123-155. doi:10.2307/2943909

Kuh, G. D. (2001). Assessing what really matters to student learning: Inside the National Survey of Student Engagement. Change, 33(3), 10-17. doi:10.1080/00091380109601795

Kuh, G. D. (2009). What student affairs professionals need to know about student engagement. Journal of College Student Development, 50, 683-706. doi:10.1353/csd.0.0099

Ladda, S. (2014). Using sport for social change: Theory into practice. Journal of Physical Education, Recreation \& Dance, 85(6), 7-11. doi:10.1080/07303084.2014.927669

Leask, B. (2005). Internationalisation of the curriculum. In J. Carroll \& J. Ryan (Eds.), Teaching international students: Improving learning for all (pp. 119-129). London, England: Routledge.

Leask, B. (2009). Using formal and informal curricula to improve the interactions between home and international students. Journal of Studies in International Education, 13, 205-221. doi:10.1177/1028315308329786

Leask, B. (2013). Internationalizing the curriculum in the disciplines_-imagining new possibilities. Journal of Studies in International Education, 17, 103-118. 
doi: $10.1177 / 1028315312475090$

Li, M. Z., \& Stodolska, M. (2006). Transnationalism, leisure, and Chinese graduate students in the United States. Leisure Sciences, 28, 39-55. doi:10.1080/01490400500332686

MacQueen, K. M., McLellan, E., Metzger, D. S., Kegeles, S., Strauss, R. P., Scotti, R., Trotter, R. T., III. (2001). What is community? An evidence-based definition for participatory public health. American Journal of Public Health, 91, 1,929-1,938. doi:10.2105/AJPH.91.12.1929

Maslow, A. H. (1943). A theory of human motivation. Psychological Review, 50, $370-396$.

Marginson, S. (2014). Student self-formation in international education. Journal of Studies in International Education, 18(1), 6-22. doi:10.1177/1028315313513036

Mathner, R. P., Martin, C. L., Tatum, S. A., \& Chouti, D. (2010). The effects of a sportsmanship education program on the behavior of college intramural sports participants. Recreational Sports Journal, 34, 119-128. doi:10.1123/rsj.34.2.119

Maxwell, J. A. (2013). Qualitative research design ( ${ }^{\text {rd }}$ ed.). Thousand Oaks, CA: Sage.

Mazon, B. K. (2010). Taking the global leap: Student affairs professionals and internationalization. Research in Comparative and International Education, 5, 205-209. doi:10.2304/rcie.2010.5.2.205

McClellan, C. (2016). Teamwork, collaboration, and cooperation as a student learning outcome for undergraduates. Assessment Update, 28(1), 5-15. doi:10.1002/au.30045

McClellan, G., King, C., \& Rockey, D., Jr. (2012). The handbook of college athletics and 
recreation administration. San Francisco, CA: Jossey-Bass.

McEwan, D., \& Beauchamp, M. R. (2014). Teamwork in sport: A theoretical and integrative review, International Review of Sport and Exercise Psychology, 7, 229-250. doi:10.1080/1750984X.2014.932423

McNaught, C., \& Lam, P. (2010). Using Wordle as a supplementary research tool. Qualitative Report, 15, 630-643. Retrieved from http://nsuworks.nova.edu/tqr/vol15/iss3/8

Mestenhauser, J. A. (1998). Portraits of an international curriculum: An uncommon multidimensional perspective. In J. A. Mestenhauser \& B. J. Elingboe (Eds.), Reforming the higher education curriculum: Internationalising the campus (pp. 339). Phoenix, AZ: American Council on Education and Oryx Press.

Mezirow, J. (Ed.) (1990). Fostering critical reflection in adulthood. San Francisco, CA: Jossey-Bass.

Mishra, N., Sharma, N., \& Kamalanabhan, T. J. (2016). Is internal communication important for sport commitment? Survey of Indian cricket players. Communication \& Sport, 4, 212-239. doi:10.1177/2167479515572810

Murff, S. H. (2005). The impact of stress on academic success in college students. The ABNF Journal, 16(5), 102-104. Retrieved from http://tuckerpub.com/abnf.htm National Association of Colleges and Employers. (2015). Professional competencies for college and university career services practictioners. Retrieved from https://www.naceweb.org/knowledge/career-services-competencies.pdf National Survey of Student Engagement. (2016). About NSSE. Retrieved from http://nsse.indiana.edu/html/about.cfm 
NIRSA: Leaders in Collegiate Recreation (NIRSA). (2015). Global Perspectives Commission. Retrieved from https://nirsa.net/nirsa/leadership/volunteer-groupsold/commissions/global-perspectives-commission/

NIRSA. (2017). NIRSA core competencies: Professional competencies for leaders in collegiate recreation. Retrieved from https://nirsa.net/nirsa/core-competencies/ Northeastern University. (2015). Mission. Retrieved from http://www.northeastern.edu/planning/mission/

Oregon State University. (2015). Mission statement. Retrieved from http://leadership.oregonstate.edu/trustees/oregon-state-university-missionstatement

Otten, M. (2003). Intercultural learning and diversity in higher education. Journal of Studies in International Education, 7(1), 12-26. doi:10.1177/1028315302250177

Ozanne, J., \& Anderson, L. (2010). Community action research. Journal of Public Policy and Marketing, 29, 123-137. doi:10.1509/jppm.29.1.123

Parsons, R. L. (2009). The effects of an internationalized university experience domestic students in the United States and Australia. Journal of Studies in International Education, 14, 313-334. doi:10.1177/1028315309331390

Perez, R. J., Shim, W., King, P. M., \& Baxter Magolda, M. B. (2015). Refining King and Baxter Magolda's model of intercultural maturity. Journal of College Student Development, 56, 759-776. doi:10.1353/csd.2015.0085

Pickering, P. M. (2006). Generating social capital for bridging ethnic divisions in the Balkans: Case studies of two Bosniak cities. Ethnic and Racial Studies, 29, 79- 
103. doi:10.1080/01419870500352397

Pieterse, A. L., Carter, R. T., Evans, S., \& Walter, R. (2010). An exploratory examination of the associations among racial and ethnic discrimination, racial climate, and trauma-related symptoms in a college student population. Journal of Counseling Psychology, 57, 255-263. doi:10.1037/a0020040

Portland State University. (2016). Vision statement. Retrieved from http://www.pdx.edu/portland-state-university-mission

Qiang, Z. (2003). Internationalization of higher education institutions. Policy Futures in Education, 1, 248-270. doi:10.1177/1028315308329804

Raynor, M. E. (1998). That vision thing: Do we need it? Long Range Planning, 31, 368-376. doi:10.1016/S0024-6301(98)80004-6

Rothwell, E., \& Theodore, P. (2006). Intramurals and college student development: The role of intramurals on values clarification. Recreational Sports Journal, 30, 46-52.

Ruderman, M., Hannum, K., Leslie, J. B., \& Steed, J. L. (2001). Making the connection: Leadership skills and emotional intelligence. Leadership in Action, 21(5), 3-7.

Ryan, G. W., \& Bernard, H. R. (2003). Techniques to identify themes in qualitative data. Field Methods, 15, 85-109. doi:10.1177/1525822X02239569

Saenz, V. B., Ngai, H. N., \& Hurtado, S. (2007). Factors influencing positive interactions across race for African American, Asian American, Latino, and White college students. Research in Higher Education, 48, 1-38. doi:10.1007/s11162-006-90263

Salas, E., Sims, D. E., \& Burke, C. S. (2005). Is there a "Big Five" in teamwork? Small Group Research, 36, 555-599. doi:10.1177/1046496405277134 
Saldaña, J. (2016). The coding manual for qualitiative researchers ( $3^{\text {rd }}$ ed.). Los Angeles, CA: Sage.

Sawir, E. (2013). Internationalisation of higher education curriculum: The contribution of international students. Globalisation, Societies and Education, 11, 359-378. doi:10.1080/14767724.2012.750477

Sawir, E., Marginson, S., Deumert, A., Nyland, C., \& Ramia, G. (2008). Loneliness and international students: An Australian study. Journal of Studies in International Education, 12, 148-180. doi:0.1177/1028315307299699

Sciarabba, D. (2012). Sports and competition in higher education: A search for values and ethics. Forum on Public Policy: A Journal of the Oxford Round Table. Retrieved from http://forumonpublicpolicy.com/vol2012.no2/archive/sciarabba.pdf

Schmitt, M. T., Spears, R., \& Branscombe, N. R. (2003). Constructing a minority group identity out of shared rejection: The case of international students. European Journal of Social Psychology, 33(1), 1-12. doi:10.1002/ejsp.131

Seifert, T. A. (2014). Student affairs and services staff in English-speaking Canadian postsecondary institutions and the role of CACUSS in professional education. Journal of College Student Development, 55, 295-309. doi:10.1353/csd.2014.0031

Shields, D. L. L., \& Bredemeier, B. J. L. (1995). Character development and physical activity. Champaign, IL: Human Kinetics Publishers. 
Shifman, R., Moss, K., Andrade, G. D., Eichel, J., \& Forrester, S. (2011). A comparison of constraints to participation in intramural sports between international and noninternational students. Recreational Sports Journal, 36, 2-12. doi:10.1123/rsj.36.1.2

Simons, H. D., Bosworth, C., Fujita, S., \& Jensen, M. (2007). The athlete stigma in higher education. College Student Journal, 41, 251-273.

Smith, R. A., \& Khawaja, N. G. (2011). A review of the acculturation experiences of international students. International Journal of Intercultural Relations, 35, 699713. doi:10.1016/j.ijintrel.2011.08.004

Snyder, B. (1971). The hidden curriculum. New York, NY: Knopf.

Söderqvist, M. (2007). Internationalisation and its management at higher-education institutions: Applying conceptual, content and discourse analysis (Doctoral dissertation, Helsinki University).

Soria, K. M., \& Troisi, J. (2013). Internationalization at home alternatives to study abroad: Implications for students' development of global, international, and intercultural competencies. Journal of Studies in International Education, 18, 261-280. doi:10.1177/1028315313496572

St. Pierre, E. A., \& Jackson, A. Y. (2014). Qualitative data analysis after coding. Qualitative Inquiry, 20, 715-719. doi:10.1177/1077800414532435

Stake, R. (1995). The art of case study research. Thousand Oaks, CA: Sage.

Stier, J. (2004). Taking a critical stance toward internationalization ideologies in higher 
education: idealism, instrumentalism and educationalism. Globalisation, Societies and Education, 2(1), 1-28. Doi:10.1080/1476772042000177069

Stier, W. F., Schneider, R. C., Kampf, S., Haines, S., \& Gaskins, B. (2008). Selected risk management policies, practices, and procedures for intramural activities at NIRSA institutions. Recreational Sports Journal, 32, 28-44. doi:10.1123/rsj.32.1.28

Stodolska, M., \& Alexandris, K. (2004). The role of recreational sport in the adaptation of first generation immigrants in the United States. Journal of Leisure Research, $36,379-413$.

Tajfel, H. (1982). Social psychology of intergroup relations. Annual Review of Psychology, 33, 1-39. doi:10.1146/annurev.ps.33.020182.000245

Tajfel, H., Brown, R., \& Turner, J. (1979). Social comparison and group interest in group favouritism. European Journal of Social Psychology, 9, 187-204. doi:10.1002/ejsp.2420090207

Tanaka, G. K. (2002). Higher education's self-reflexive turn: Toward an intercultural theory of student development. Journal of Higher Education, 73, 263-296. doi:10.1353/jhe.2002.0024

Tierney, W. G. (1988). Organizational culture in higher education: Defining the essentials. Journal of Higher Education, 59, 2-21. doi:10.2307/1981868

Tierney, W. G. (1992). An anthropological analysis of student participation in college. Journal of Higher Education, 63, 603-618. doi:10.1080/00221546.1992.11778391

Tinto, V. (1975). Dropout from higher education: A theoretical synthesis of recent research. Review of Educational Research, 45, 89-125. 
doi:10.3102/00346543045001089

Trowler, V. (2010). Student engagement literature review. Retrieved from the Higher Education Academy website: https://www.heacademy.ac.uk/resources/detail/evidencenet/Student_engagement_ literature_review

Twombly, S., Salisbury, M., Tumanut, S., \& Klute, P. (2012). Special issue: Study abroad in a new global century-Renewing the promise, refining the purpose. ASHE Higher Education Report, 38(4), 1-152. doi:10.1002/aehe.20004

University of Oregon. (2015). Vision statement. Retrieved from http://pages.uoregon.edu/uosenate/UOmissionstatement.html

Urban, E. L. (2012). Value of U.S. higher education for international students in the context of higher education internationalization (Doctoral dissertation). Retrieved from ProQuest Dissertations \& Theses Global database. (UMI No. 3536231)

Urban, E. L., \& Orbe, M. P. (2007). “The syndrome of the boiled frog:” Exploring international students on U.S. campuses as co-cultural group members. Journal of Intercultural Communication Research, 36, 117-138.

doi:10.1080/17475750701478695

Urban, E. L., \& Palmer, L. (2014). International students as a resource for internationalization of higher education. Journal of Studies in International Education, 18, 305-324. doi:10.1177/1028315313511642

Vincent, M., \& Kearney, J. (2001). Monitoring and promoting good sportsmanship as an essential element of intramural sports. Recreational Sports Journal, 25, 34-41. 
doi:10.1123/nirsa.25.1.34

Vygotsky, L. S. (1978). Mind in society: The development of higher psychological processes. Cambridge, MA: Harvard University Press.

Wachter, B. (2003). An introduction: Internationalization at home in context. Journal of Studies in International Education, 7(1), 5-11. doi:10.1177/1028315302250176

Walseth, K., \& Fasting, K. (2004). Sport as a means of integrating minority women. Sport in Society, 7, 109-129. doi:10.1080/1461098042000220218

Wann, D. L., \& Grieve, F. G. (2005). Biased evaluations of in-group and out-group spectator behavior at sporting events: The importance of team identification and threats to social identity. Journal of Social Psychology, 145, 531-545. doi:10.3200/SOCP.145.5.531-546

Ward, C. (2001). The impact of international students on domestic students and host institutions (Report prepared for the Export Education Policy Project of the New Zealand Ministry of Education). Retrieved from http://www.educationcounts.govt.nz/publications/international/14684

Weiss, O. (2001). Identity reinforcment in sport: Revisiting the symbolic interactionist theory. International Review for the Sociology of Sport, 36, 393-405. doi:10.1177/101269001036004002

Yoh, T., Gordon, B., \& Yang, H. (2008). Status of participation in physical activities among international students attending colleges and universities in the United States. College Student Journal, 42, 1,110-1,117. 
Appendix A

NIRSA Core Competencies

\section{Programming}

Program delivery to our communities is a core purpose for collegiate recreation professionals, and often appears in departmental and divisional missions. Although specific programs may vary from one institution to another, professionals need to be able to develop and administer high quality programs and to appropriately apply associated resources.

\section{Philosophy \& Theory}

The Philosophy \& Theory competency area involves knowledge and skills that connect the philosophy and theory to professional practice. Informing our practice through the use of existing frameworks and development of new frameworks provides the context for best practices. As we operate in a higher education environment, our work gives us the high-powered opportunity to apply the process of praxis.

\section{Personal \& Professional Qualities}

The Personal \& Professional Qualities competency area involves the knowledge, skills, and attitudes to inform individual conduct and apply these tools toward productive work and interaction; awareness of one's own areas of strengths and areas for development; be committed to lifelong learning; to assimilate integrity and ethics into all aspects of personal and professional self.

\section{Legal Liabilities \& Risk Management}

With the inherent risk involved with collegiate recreation activities and the litigious nature of our society, collegiate recreation professionals must be able to identify and manage risks. With experience, professionals will need to be able to understand liability and manage risk at more sophisticated levels.

\section{Human Resources Management}

The Management Techniques competency area involves the knowledge, skills and abilities to be an effective supervisor/ manager for human resources; to create a productive workplace with efficient policies and procedures and to train and develop staff.

\section{Facility Management, Planning \& Design}

Facility Management in the field of campus recreation covers a broad scope of skill sets needed to successfully operate, maintain and plan new attractive, safe and fully operable 
built environments. Administration, facility operations, energy/utilities management, building design and construction are essential skills at every level of employment.

\section{Business Management}

Business Management in the field of campus recreation covers a broad scope of skill sets needed across disciplines within our field. Communicating, planning, marketing, managing resources, strategic relationship building, and utilizing appropriate technology are essential business management skills at every level of employment.

\section{Research \& Evaluation}

Accountability in higher education is achieved through data-informed decisions and objective reporting of program outcomes. As a profession, collegiate recreation must continue generating research that increases knowledge that advances the profession. The general trend of increasing complexity of knowledge and skills in research and evaluation shows a progression from being able to participate, interpret, and apply findings to being able to conceive of and direct research, assessment, and evaluation processes. At the more complex level, one should be able to direct others in conducting research, assessment, and evaluation activities, participate in more sophisticated studies, conduct meta analyses of multiple data sets, and communicate results to garner program support and resources. 
Table A.1

NIRSA Professional Competencies for Programming - Selected Implications

\begin{tabular}{|c|c|c|c|}
\hline Category & Basic & Intermediate & Advanced \\
\hline $\begin{array}{l}\text { Student } \\
\text { Learning and } \\
\text { Development }\end{array}$ & $\begin{array}{l}\text { Articulate theories and } \\
\text { models that describe the } \\
\text { development of college } \\
\text { students and the conditions } \\
\text { and practices that facilitate } \\
\text { holistic development. }\end{array}$ & $\begin{array}{l}\text { Design programs, facilities, } \\
\text { and services to promote } \\
\text { student learning and } \\
\text { development that are based } \\
\text { on current research on } \\
\text { student learning and } \\
\text { development theories. }\end{array}$ & $\begin{array}{l}\text { Develop staff members' } \\
\text { competency in applying } \\
\text { learning and development } \\
\text { theory to practice. }\end{array}$ \\
\hline $\begin{array}{l}\text { Teaching and } \\
\text { Training }\end{array}$ & $\begin{array}{l}\text { Integrate various learning } \\
\text { theories and models into } \\
\text { training and programming } \\
\text { practices. }\end{array}$ & $\begin{array}{l}\text { Design opportunities for } \\
\text { student training and } \\
\text { program development that } \\
\text { encourage continual } \\
\text { learning and } \\
\text { developmental growth. }\end{array}$ & $\begin{array}{l}\text { Reinforce and assess } \\
\text { inclusive and welcoming } \\
\text { campus communities that } \\
\text { promote deep learning and } \\
\text { foster student success. }\end{array}$ \\
\hline $\begin{array}{l}\text { Program } \\
\text { Development }\end{array}$ & $\begin{array}{l}\text { Align programs with } \\
\text { departmental/institutional } \\
\text { goals, missions, and } \\
\text { priorities. }\end{array}$ & $\begin{array}{l}\text { Use intentional planning } \\
\text { methods that include } \\
\text { development of student } \\
\text { learning outcomes. }\end{array}$ & \\
\hline Leadership & $\begin{array}{l}\text { Identify basic teamwork } \\
\text { fundamentals and } \\
\text { teambuilding strategies in } \\
\text { one's work setting and } \\
\text { communities of practice. }\end{array}$ & $\begin{array}{l}\text { Create environments that } \\
\text { encourage students to view } \\
\text { themselves as having the } \\
\text { potential to make } \\
\text { meaningful contributions } \\
\text { to their communities and to } \\
\text { be engaged in their } \\
\text { communities. }\end{array}$ & $\begin{array}{l}\text { Foster an institutional } \\
\text { culture that supports the } \\
\text { free and open exchange of } \\
\text { ideas and beliefs, and } \\
\text { where issues of power and } \\
\text { privilege are identified and } \\
\text { addressed. }\end{array}$ \\
\hline $\begin{array}{l}\text { Equity, } \\
\text { Diversity and } \\
\text { Inclusion }\end{array}$ & $\begin{array}{l}\text { Participate in activities that } \\
\text { challenge one's beliefs. } \\
\text { Design culturally relevant } \\
\text { and inclusive programs, } \\
\text { services, policies, and } \\
\text { practices. }\end{array}$ & $\begin{array}{l}\text { Integrate cultural } \\
\text { knowledge with specific } \\
\text { and relevant cultural issues } \\
\text { on campus. }\end{array}$ & $\begin{array}{l}\text { Ensure that competence in } \\
\text { equity, diversity, and } \\
\text { inclusion is fully integrated } \\
\text { into departmental practices } \\
\text { throughout the campus. }\end{array}$ \\
\hline $\begin{array}{l}\text { Global } \\
\text { Perspective }\end{array}$ & $\begin{array}{l}\text { Predict access needs for } \\
\text { potential participants. } \\
\text { Develop one's own civic } \\
\text { capacity (such as a sense of } \\
\text { personal and social } \\
\text { responsibility to understand } \\
\text { the connection between a } \\
\text { global perspective and how } \\
\text { people relate to one } \\
\text { another). }\end{array}$ & $\begin{array}{l}\text { Foster and implement } \\
\text { programming that creates a } \\
\text { sense of personal and } \\
\text { social responsibility in } \\
\text { others to develop students } \\
\text { across multiple } \\
\text { dimensions. }\end{array}$ & $\begin{array}{l}\text { Analyze the } \\
\text { interconnectedness of } \\
\text { societies worldwide and } \\
\text { how these global } \\
\text { perspectives affect } \\
\text { institutional learning and } \\
\text { make connections to } \\
\text { collegiate recreation. }\end{array}$ \\
\hline & & & $\begin{array}{l}\text { Apply the dimensions of } \\
\text { personal and social } \\
\text { responsibility. }\end{array}$ \\
\hline
\end{tabular}


Table A. 2

NIRSA Professional Competencies for Philosophy and Theory - Selected Implications

\begin{tabular}{|c|c|c|c|}
\hline Category & Basic & Intermediate & Advanced \\
\hline $\begin{array}{l}\text { Student Affairs } \\
\text { Issues and } \\
\text { Theories }\end{array}$ & $\begin{array}{l}\text { Identify issues and } \\
\text { theories in the broader } \\
\text { field of student affairs } \\
\text { and be aware of their } \\
\text { significance in } \\
\text { collegiate recreation. }\end{array}$ & $\begin{array}{l}\text { Facilitate discussion on } \\
\text { issues and theories in the } \\
\text { broader field of student } \\
\text { affairs and make connections } \\
\text { to their significance in } \\
\text { collegiate recreation. }\end{array}$ & $\begin{array}{l}\text { Assess departmental staff's } \\
\text { praxis on issues and theories } \\
\text { in the broader field of student } \\
\text { affairs and how they make } \\
\text { connections to their } \\
\text { significance in collegiate } \\
\text { recreation. }\end{array}$ \\
\hline $\begin{array}{l}\text { Student } \\
\text { Development } \\
\text { Theory } \\
\text { (Participants/E } \\
\text { mployees) }\end{array}$ & $\begin{array}{l}\text { Demonstrate theories } \\
\text { and frameworks which } \\
\text { inform the work in } \\
\text { collegiate recreation on } \\
\text { the following topics s: } \\
\text { Student development } \\
\text { (employees/participant } \\
\text { s); engagement; } \\
\text { participant based; } \\
\text { Health \&Wellness; } \\
\text { Equity \& Diversity; } \\
\text { Leadership; } \\
\text { Sustainability; Global } \\
\text { Perspective and } \\
\text { Service. }\end{array}$ & $\begin{array}{l}\text { Apply theories and } \\
\text { frameworks in university } \\
\text { recreation on topics such as: } \\
\text { Student development/ } \\
\text { engagement } \\
\text { (employees/participants); } \\
\text { participant based; Health } \\
\text { \&Wellness; Equity \& } \\
\text { Diversity; Leadership; } \\
\text { Sustainability; Global } \\
\text { Perspective and Service. } \\
\text { Incorporate philosophies and } \\
\text { theories from student affairs } \\
\text { and university recreation into } \\
\text { staff training and } \\
\text { development at the student } \\
\text { and professional staff levels. }\end{array}$ & $\begin{array}{l}\text { Develop and promote new } \\
\text { frameworks in university } \\
\text { recreation on topics such as: } \\
\text { Student } \\
\text { development/engagement } \\
\text { (employees/ participants); } \\
\text { participant based; Health } \\
\& \text { Wellness; Equity \& } \\
\text { Diversity; Leadership; } \\
\text { Sustainability; Global } \\
\text { Perspective and Service. }\end{array}$ \\
\hline \multirow[t]{2}{*}{$\begin{array}{l}\text { Emerging } \\
\text { Trends in } \\
\text { Recreational } \\
\text { Sports }\end{array}$} & $\begin{array}{l}\text { Be aware of past and } \\
\text { present trends that } \\
\text { have } \\
\text { influenced practices in } \\
\text { university recreation. }\end{array}$ & $\begin{array}{l}\text { Monitor emerging trends and } \\
\text { issues that have } \\
\text { the potential to influence } \\
\text { practices in university } \\
\text { recreation. }\end{array}$ & $\begin{array}{l}\text { Evaluate emerging trends and } \\
\text { execute implementation to } \\
\text { ensure timely and relevant } \\
\text { information and services to } \\
\text { the institution. }\end{array}$ \\
\hline & & $\begin{array}{l}\text { Plan and implement new } \\
\text { programs and services } \\
\text { to address emerging trends } \\
\text { and issues at the } \\
\text { institutional level. }\end{array}$ & $\begin{array}{l}\text { Provide leadership to } \\
\text { departmental, divisional or } \\
\text { institutional colleagues by } \\
\text { presenting information } \\
\text { on emerging trends. }\end{array}$ \\
\hline $\begin{array}{l}\text { Equity, } \\
\text { Diversity, and } \\
\text { Inclusion }\end{array}$ & $\begin{array}{l}\text { Examine issues of } \\
\text { equity and diversity } \\
\text { and be } \\
\text { aware of their } \\
\text { significance in } \\
\text { collegiate recreation. }\end{array}$ & $\begin{array}{l}\text { Teach staff and student } \\
\text { employees about issues } \\
\text { of equity and diversity and } \\
\text { demonstrate their } \\
\text { significance in collegiate } \\
\text { recreation. }\end{array}$ & $\begin{array}{l}\text { Advocate the adoption of } \\
\text { practices that support } \\
\text { and enhance equity and } \\
\text { diversity and promote their } \\
\text { significance in collegiate } \\
\text { recreation. }\end{array}$ \\
\hline
\end{tabular}


Table A.3

NIRSA Professional Competencies for Personal and Professional Qualities - Selected Implications

\begin{tabular}{|c|c|c|c|}
\hline Category & Basic & Intermediate & Advanced \\
\hline \multirow[t]{3}{*}{ Adaptability } & $\begin{array}{l}\text { Demonstrate effectiveness } \\
\text { when changes occur in } \\
\text { policies, procedures, } \\
\text { culture, situations, and } \\
\text { work assignments. }\end{array}$ & $\begin{array}{l}\text { Support and seek } \\
\text { opportunities for change } \\
\text { that show the promise of } \\
\text { improving established } \\
\text { processes. }\end{array}$ & $\begin{array}{l}\text { Anticipate change and make } \\
\text { significant or long-term } \\
\text { adaptations in organization } \\
\text { in response to the needs of } \\
\text { the situation. }\end{array}$ \\
\hline & $\begin{array}{l}\text { Integrate input from staff } \\
\text { and stakeholders to adjust } \\
\text { project plans. }\end{array}$ & $\begin{array}{l}\text { Assess feedback for } \\
\text { consideration while } \\
\text { implementing change. }\end{array}$ & $\begin{array}{l}\text { Identify the contrast } \\
\text { between macro-strategic } \\
\text { issues and critical details. }\end{array}$ \\
\hline & & & $\begin{array}{l}\text { Evaluate alternatives, and } \\
\text { respond quickly and } \\
\text { effectively to unexpected } \\
\text { and rapidly changing } \\
\text { conditions. }\end{array}$ \\
\hline \multirow[t]{2}{*}{$\begin{array}{l}\text { Equity } \\
\text { Diversity and } \\
\text { Inclusion }\end{array}$} & $\begin{array}{l}\text { Attend diversity programs } \\
\text { to increase staff } \\
\text { awareness }\end{array}$ & $\begin{array}{l}\text { Recognize and utilize the } \\
\text { skills of staff with diverse } \\
\text { backgrounds to benefit the } \\
\text { organization, clients, and } \\
\text { coworkers. }\end{array}$ & $\begin{array}{l}\text { Recognize and utilize the } \\
\text { skills of staff with diverse } \\
\text { backgrounds to benefit the } \\
\text { organization, clients, and } \\
\text { coworkers. }\end{array}$ \\
\hline & & $\begin{array}{l}\text { Address and correct the } \\
\text { use of inappropriate } \\
\text { language or actions that } \\
\text { denigrate diversity. }\end{array}$ & $\begin{array}{l}\text { Address and correct the use } \\
\text { of inappropriate language or } \\
\text { actions that denigrate } \\
\text { diversity. }\end{array}$ \\
\hline \multirow[t]{3}{*}{$\begin{array}{l}\text { Global } \\
\text { Perspective }\end{array}$} & $\begin{array}{l}\text { Differentiate and respect } \\
\text { other cultural perspectives } \\
\text { and norms. }\end{array}$ & $\begin{array}{l}\text { Facilitate interaction } \\
\text { across global and } \\
\text { international differences } \\
\text { in areas such as language }\end{array}$ & $\begin{array}{l}\text { Ensure that policies and } \\
\text { procedures serve a diverse } \\
\text { and multicultural base. }\end{array}$ \\
\hline & $\begin{array}{l}\text { Seek experiences in } \\
\text { multi-cultural } \\
\text { environments. } \\
\text { Develop awareness of } \\
\text { international differences. }\end{array}$ & $\begin{array}{l}\text { and communication; } \\
\text { knowledge of traditional } \\
\text { and popular culture; } \\
\text { business and employment } \\
\text { practices and by following } \\
\text { the news in other } \\
\text { countries. }\end{array}$ & $\begin{array}{l}\text { Distinguish cultural } \\
\text { differences and incorporate } \\
\text { concepts into programming } \\
\text { and/or facility design and } \\
\text { operations to educate about } \\
\text { the differences. }\end{array}$ \\
\hline & $\begin{array}{l}\text { Prepare to communicate } \\
\text { across cultural and } \\
\text { linguistic boundaries. }\end{array}$ & $\begin{array}{l}\text { Connect study abroad } \\
\text { programs by infusing } \\
\text { recreational activities with } \\
\text { cultural and educational } \\
\text { opportunities. }\end{array}$ & \\
\hline
\end{tabular}


Appendix B

Fall 2016 Research Timeline

Event

Document Review

Intervention A

Intramural Staff Training

Teams Signed Up

Intervention B

Team Captain Meeting//Staff Training

Intervention $\mathrm{C}$

Games

Intervention D

IM Social for Players and Staff

Observation Window

Distribute Electronic Survey - Includes

Informed Consent for Players

Distributed Reminder to Complete Survey

Closed Survey

Initial Review of Survey Answers

Scheduled In-Depth Interviews

Conducted In-Depth Interviews
Date

September 12-16

September 19-23

September 26 - October 6

October 6

October 13 - November 26

October 20 and October 23

October 13 - November, 26

November 11

November 16

November 20, midnight

November 21-22

November 22-27

November 28 - January 2017 
Appendix C

Global-Self Training for Intramural Staff

Classroom Session

1. Intro - 10 minutes (possible videos to include below)

a. Video from the Copa America referencing Trump's building a wall to keep "them" out: hpps://www.youtube.com/watch?v=sTEnBC-2ZL4

b. Soccer globalization 101: https:/www.youtube.com/watch?v=UxL5_Ck0jM

c. Summary of How Soccer Explains the World: https://www.youtube.com/watch?v=8Amo1b3BUgc

2. Staff Introductions -15 minutes

a. What are the backgrounds of the staff?

b. Why are they interested in soccer?

3. Introduction to Internationalization and Globalization - 15-Minute Lecture

a. Definitions of internationalization and globalization

b. Review data on national and international student movement

i. Review Open Doors data sets

ii. Review Portland State international student demographics for school, department, program

c. University Strategic Plan:

i. 7.4 Create opportunities for international and intercultural engagement in existing campus housing, cultural and recreation programs. 
d. Review NIRSA Global Perspective Value

i. http://nirsa.net/nirsa/leadership/volunteergroups/commissions/global-perspectives-commission/

ii. Actively answer questions

4. Sports for Social Change -10 minutes

a. Data on participants worldwide - focusing on soccer attributes and global engagement

b. Review section from proposal - both historically and locally

c. Hitler Olympics, John Carlos, World Cup

5. Global-Self: What are the self-in-the-world and act-in-the-world attributes Reflective Activities and Conversation - 20 minutes

a. Staff reflect on what they think of when they think of having a global perspective or being a global citizen

b. The others with whom we interact are equally human

i. What does that mean: "Global students and global selves need an equally skeptical stance towards their own ways of being as they have towards those of others"

c. Skills of act-in-the-world

i. What are the tools you identify as needing?

ii. Language, conflict resolution, cultural awareness

6. Specific cultural training from guest speakers for major international student groups - partnership with IELP and International Student Services: 30 minutes

a. Chinese. Kuwaiti. Japanese. Brazilian. 
Intramural Staff Hands-On Training Session

1. Pre-Game Scripts

a. Introducing the concept of Fair Play

b. Introducing the Rules-of-the-Game

i. Rule modifications for Fair Play

ii. Soccer Penalty Boxes

iii. Sportsmanship Ratings

c. Reminder of Sportsmanship Prizes/Penalties

2. Pre-Game Activities

a. Whole group introductions

i. Name, Year-in-school, Major, Why you play

1. These attributes should not induce "us-vs-them"

3. Introduction of Post-Game Activities

a. Handshakes

b. Sportsmanship Evaluations

i. Each team evaluates themselves and the other team and IM staff

ii. Use technology to do this and include questions that prompt global thinking 
Appendix D

\author{
Pre-Game Script and Questions \\ Referee Script to the Whole Team
}

8V8 Outdoor Soccer

Fall 2016

Require the whole team to come to midfield

- 18-minute halves with a 2-minute halftime

- Safety is our first concern

- Absolutely no slide tackling

- Keepers can dive but only sideways

- Yellow card penalty of two minutes

- Sportsmanship ratings influence your ability to participate in playoffs

- Each team starts with a 5 and gets points deducted for things including cards, language, cursing, and harassment of any kind including sexism, racism or harassment based on nationality

The best formula for sportsmanship is by demonstrating fair play and respect. At Portland State intramurals, we believe soccer can be used to make this world a better place in which everyone can live. We use intramurals to promote health and inclusion for everyone. To help us accomplish this, please introduce yourself to each other and the referees by saying your name, where you are from, and ...

- Week 1 - Your favorite Olympic sport

- Week 2 - The favorite place you have traveled

- Week 3 - How many friends do you have that are from different countries than yours?

- Week 4 - Has anyone ever stereotyped you based on where you are from?

- Week 5 - What is something you've learned about other people on different teams?

- Week 6 - What is your favorite holiday that you celebrate? 
Appendix E

Questions for Post-Game Interventions

$\underline{\text { Post-Game Intervention One }}$

Seven different questions, printed out on thin strips of paper, were handed out randomly to participants on each team after the game. Each handout had one of the following statements on it:

To get a piece of pizza find a person from the other team and find the/their answer to the following question:

One of the following seven questions followed the directions:

Where in the world is Saint Kitts and Nevis?

Where in the world are the Maldives?

Where in the world is Tunisia?

What is your favorite national holiday?

What is your favorite national celebration in your country?

What is your favorite family holiday?

Where in the world is your favorite food from and what is it?

\section{Post-Game Intervention Two}

The second post-game introduction was modified by narrowing down the scope of the questions and providing more context for the activity. The questions were handed out to players before the game to prime them to both think about and discuss the questions posed.

Halloween treats are available for you after the game today! All you have to do to get your treat is to find a player from the team that you play against today and come with them to the tent and try to answer one of the following questions (you don't even need to be right):

- What is your favorite national celebration in your country and how would you describe it?

- Where is Diwali celebrated and what is it?

- Where in the world did Halloween originate and why?

- What is Día de los Muertos and where did it originate? 


\section{Appendix F}

\section{Protocol for Online Survey}

The protocol followed to get participant replies to my on-line survey was:

First, I sent an email to all participants in the intramural program:

Thank you for participating in Portland State's intramural soccer program. My name is Alex Accetta and I am a doctoral student in the School of Education. My dissertation research examines students' perceived development of their globalself when they participate in an intentionally designed intramural soccer program. Part of my research involves asking participants to respond to survey questions about their experiences in this year's intramural season. Respondents who complete the survey will receive a $\$ 10$ gift-card to the University Market in Smith Student Union. To access the survey, click here:

[insert Qualtrics web-site address].

When the participant clicked on the link they were directed to a Qualtrics survey that included the following:

\section{Introduction}

Thank you for taking the time to respond to my survey on intramural soccer and the development of the global-self. This survey is intended to understand your experience in this quarter's intramural soccer season and how it impacted your perceptions of the development of your global citizenship.

Section One was the Informed Consent Form.

Section Two included the following questions:

1. What about soccer helps you think about others in a global context? 
2. What does it mean to you for someone to have a global perspective?

3. What is your view of your own global perspective?

4. Describe the way that participants (players and staff) in the intramural soccer league treat each other.

5. Thinking of the soccer season, describe your interactions with players or intramural staff that may have had a different background than you, particularly focusing on people from different cultures.

6. What type of changes occurred for you during the soccer season in how you interacted with people who were different than you?

7. Demographics: Gender, Year in School, Country of Citizenship, Racial Identity

Section Three was disassociated from Section Two via a separate survey link

1. Email address to receive gift card

2. Consent to be contacted for interview 


\author{
Appendix G \\ Informed Consent Form \\ The Portland State University \\ Consent to Participate in Research \\ Development of the Global-Self through Collegiate Recreational Sports
}

November 11, 2016

\title{
Introduction
}

You are being asked to participate in a research study that is being done by Alex Accetta, who is the Principal Investigator from the Department of Education, at Portland State University in Portland, Oregon. This research is studying how students perceive they develop their global-self after participating in intramural soccer.

You are being asked to participate in this study because you are participating in the intramural soccer program in the Fall of 2016.

This form will explain the research study, and will also explain the possible risks as well as the possible benefits to you. We encourage you to talk with your family and friends before you decide to take part in this research study. If you have any questions, please ask one of the study investigators.

\section{What will happen if I decide to participate?}

If you agree to participate, the following things will happen:

You will take a seven question on-line questionnaire. Based on the results of the questionnaire you may be asked to participate in an in-person interview.

\section{How long will I be in this study?}

Participation in this initial section of the study will take a total of approximately 20 minutes. If you are selected and choose to participate in the interview process the interview may take approximately 1 hour.

\section{What are the risks or side effects of being in this study?}

There are minimal risks of stress, emotional distress, inconvenience associated with participating in a research study.

\section{What are the benefits to being in this study?}


There is potentially no direct benefit to the subject. By participating in the study, however, you will have the opportunity to reflect on your experience during intramural soccer this quarter. The researcher does plan to share the results of the study through educational conferences to improve the performance of intramural staff throughout the country.

\section{How will my information be kept confidential?}

We will take measures to protect the security of all your personal information. The raw qualitative data gathered will be kept confidential and any personal identifiers will be removed and destroyed as soon as possible. Your name will not be used in any published reports about this study. As part of the data cleaning, confidentially will be addressed by the use of pseudonyms for any written report or verbal presentation. The informed consent forms will be appropriately documented and measures will be taken to protect the security of all personal information including locking all research material behind two locks when not being used. The on-line data will be stored on a secure Portland State server.

Information contained in your study records is used by the researcher. The Portland State University Institutional Review Board (IRB) that oversees human subject research may be permitted to access your records, and there may be times when we are required by law to share your information. It is the investigator's legal obligation to report child abuse, child neglect, elder abuse, harm to self or others or any life-threatening situation to the appropriate authorities, and; therefore, your confidentiality will not be maintained.

\section{Will I be paid for taking part in this study?}

Each person who completes the following on-line questionnaire will receive a \$10 gift card to the University Market. Each person who agrees to participate in the interview process will receive a \$20 gift card to the University Market.

\section{Can I stop being in the study once I begin?}

Your participation in this study is completely voluntary. You have the right to choose not to participate or to withdraw your participation at any point in this study without penalty or loss of benefits to which you are otherwise entitled.

\section{Whom can I call with questions or complaints about this study?}

If you have any questions, concerns or complaints at any time about the research study, please contact Alex Accetta at 503-725-8787 or Dr. Karen Haley 503-725-8270

\section{Whom can I call with questions about my rights as a research participant?}


If you have questions regarding your rights as a research participant, you may call the PSU Office for Research Integrity at (503) 725-2227 or 1 (877) 480-4400. The ORI is the office that supports the PSU Institutional Review Board (IRB). The IRB is a group of people from PSU and the community who provide independent oversight of safety and ethical issues related to research involving human participants. For more information, you may also access the IRB website at https://sites.google.com/a/pdx.edu/research/integrity

\section{CONSENT}

You are making a decision whether to participate in this study. Your signature below indicates that you have read the information provided (or the information was read to you). By signing this consent form, you are not waiving any of your legal rights as a research participant.

You have had an opportunity to ask questions and all questions have been answered to your satisfaction. By signing this consent form, you agree to participate in this study. A copy of this consent form will be provided to you.

$\overline{\text { Name of Adult Subject (print) Signature of Adult Subject }}$ Date

\section{INVESTIGATOR SIGNATURE}

This research study has been explained to the participant and all of his/her questions have been answered. The participant understands the information described in this consent form and freely consents to participate.

Name of Investigator / Research Team Member (type or print)

(Signature of Investigator / Research Team Member) Date 
Appendix $\mathrm{H}$

Online Survey

Global Self Through Collegiate Recreation

Q1

Thank you for taking the time to respond to my survey on intramural soccer and the development of the global-self.

This survey is intended to understand your experience in this quarter's intramural soccer season and how it impacted your perspective of the development of your global self. There are two components to the global self: 1) The knowing of your self-in-the-world, including the understanding that other people with whom you exist are equally human.2) The knowledge, skills, and values which enable you to act-in-the-world with appropriate references to the context and participants in your interactions.

Q2

Consent Form Introduction You are being asked to participate in a research study that is being done by Alex Accetta, who is the Principal Investigator from the Department of Education, at Portland State University in Portland, Oregon. This research is studying how students perceive they develop their global-self after participating in intramural soccer. You are being asked to participate in this study because you are participating in the intramural soccer program in the Fall of 2016. This form will explain the research study, and will also explain the possible risks as well as the possible benefits to you. We encourage you to talk with your family and friends before you decide to take part in this research study. If you have any questions, please ask one of the study investigators. By clicking to continue, you agree to participate in this study. You may print out a copy of this form for your records.

Participate and Continue (1)

Opt-Out (2)

If Opt-Out Is Selected, Then Skip to End of Survey

Q3

Are you an...

Intramural Player (1)

Intramural Staff (2)

If Intramural Player Is Selected, Then Skip to During the intramural... 
Q4

During the intramural season the intramural program introduced a number of methods to help students interact with each other, including training our staff on the global self, the captains' meeting, the way the staff led and managed the games (including your training, the pre-game introductions, and the questions for getting pizza and/or Halloween candy). Keeping these in mind, please answer the following questions.

Q5

If you attended staff training, describe your own global perspective and the development of your global self was, or was not, impacted by the staff training.

Q6

During the season, you used methods to help players and staff get to know each other. Please describe how these methods impacted the sportsmanship and interactions among players and between players and staff.

Q7

Thinking of the intramural soccer season, describe your interactions with players or intramural staff that may have had a different background than you.

Q8

Through your employment in intramural soccer, please describe any knowledge, skills, or values you learned about working with other people, including those different than you.

Q9

Thinking of your work and training this intramural soccer season, describe any new insights you had about the idea that the others in the league are equally human.

Q10

Describe your experience implementing the interventions we used to encourage staff and players to get to know each other. The interventions included intramural staff training, the captains' meeting, meeting, the questions for pizza and/or Halloween candy, and the game management techniques we introduced (including the pre-game introductions and questions).

Q11

Describe your overall experience with the intramural season.

If Describe your overall experience... Is Not Empty, Then Skip to What gender do you identify with? 
Q12

During the intramural season the intramural program introduced a number of methods to help students interact with each other, including training our staff on the global self, the captains' meeting, questions for pizza and/or Halloween candy, and the game management techniques we introduced including pre-game introductions and questions. Keeping these in mind, please answer the following questions:

Q13

Thinking of the soccer season, describe your interactions with players or intramural staff that had a different background than you.

Q14

What were your reactions to the strategies we used to lead and manage the games, especially thinking about how the staff attempted to help people get to know each other through the pizza/Halloween questions or the pre-game introductions and questions?

Q15

What type of changes occurred for you during the soccer season in how you interacted with people with people?

Q16

Through your participation in intramural soccer, please describe any knowledge, skills, or values you learned about working with other people, including those different than you.

Q17

Thinking of the intramural soccer season, describe any new insights you had about the idea that the others participating and working in the league are equally human.

Q18

How did your experience of intramural soccer change how you viewed the players on other teams and/or how you viewed the staff?

Q19

Describe your overall experience with the intramural season.

If Describe your overall experience... Is Not Empty, Then Skip to What gender do you identify with?

Q20

What gender do you identify with?

O Male (1)

Female (2)

Transgender (3) 
Q21

What year are you in school?

Freshman (1)

Sophomore (2)

O Junior (3)

O Senior (4)

O Graduate Student (5)

O Post-bac (6)

Q22

What is your country of citizenship?

Q23

What is your racial identity?

Q24

Thank you for completing this survey! Would you be willing to be interviewed in person about this topic? Selected participants will receive a \$20 thank-you card.

Y Yes (Opens another survey to enter your contact information to be eligible for the $\$ 10$ thank-you card and potentially for a \$20 thank-you card if you are interviewed. This information is not linked to your current responses.) (1)

No (Opens another survey to enter your information to be eligible for the $\$ 10 \mathrm{gift}$ card. The information is not linked to your current responses) (2) 
Appendix I

Semi-Structured Interview Protocol

This was the protocol for the semi-structured interviews with soccer players and intramural staff.

\section{For players:}

Greeting and read the purpose of the study.

1) Tell me a little bit about yourself, what soccer means to you, and how you came to play/staff the sport.

2) Share with me your understanding of how soccer impacts the way people around the world view each other.

3) How do you think your prior experience impacted how you interacted with the other intramural players during the season?

4) Share with me how you think soccer impacts the way you view others, particularly focusing on those different from you.

a. Prompt for racial, gender, and nationality differences

Reflecting on the interactions you had with the intramural players, staff, and referees:

5) Describe how the game management methods impacted how you interacted with players from the other team.

a. Prompts should include pre-game script, introductions, social, referees

b. Prompt should include an emphasis on the other players specifically

6) How do you think the game management methods impacted how you interacted with the staff and the referees?

c. Prompts should include pre-game script, introductions, social, referees 
7) How do you think your global perspective has changed as a result of your participation in intramural soccer?

\section{For staff:}

Greeting and read the purpose of the study.

1) Tell me a little bit about yourself, what soccer means to you, and how you came to play/staff the sport.

2) Share with me your understanding of how soccer impacts the way people around the world view each other.

3) How do you think your prior experience impacted how you interacted with the intramural players?

4) Share with me how you think soccer impacts the way you view others, particularly focusing on those different from you.

a. Prompt for racial, gender, and nationality differences

Reflecting on your training and your experience working intramurals this quarter:

5) How do you think the staff training impacted your interactions with the intramural players?

6) What did you observe about how the interventions we used impacted the players' attitude towards each other and the referees?

a. Prompt for training, captains' meeting, game intervention, social intervention

7) What were your observations about others' global-self over the course of the whole intramural season?

a. Prompt for conversations they heard/had 
b. Prompt for behavior they noticed

8) What were your observations about your global-self over the course of the whole intramural season from staff training to completion?

a. Prompt for where they thought they were before the training

b. Prompt for anything they thought might have impacted them in the training

c. Prompt for where they perceive their global-self is at the completion of the season 
Appendix $\mathbf{J}$

\section{Survey Response Demographics}

When the survey closed, 49 players and five staff, or $19.15 \%$ of the participants and staff, had completed the survey. Of these 54 total responses, 40 were males and 14 were females; ; 11\% were freshman, 11\% were sophomores, $19 \%$ were juniors, $26 \%$ were seniors, and $25 \%$ were graduate or post-baccalaureate students. There were 33 students that identified that they were from the United States. Sixteen students identified that they were from other countries including: Columbia, Canada, India, Vietnam, France, Saudi Arabia, China, Mexico, Algeria, and Kuwait. One student said they were from earth. 